\title{
INTEGRAL DE RIEMANN VETORIAL E \\ GEOMETRIA DE ESPACOS DE BANACH
}

GALDINO CESAR DA ROCHA FILHO

TESE APRESENTADA

A0

INSTITUTO DE MATEMATICA E ESTATISTICA

DA

UNIVERSIDADE DE SĀO PAULO

PARA OBTENÇĀO DO GRAU DE DOUTOR

EM

MATEMATICA

ORIENTADOR:

Prof. Dr. Chaim Samuel Hönig

- SĀo paulo, outubro de 1979 - 



\section{AGRADECIMENTOS}

Ao meu orientador, Professor Doutor Chaim Samuel Hönig, minha gratidão. Em seus trabalhos se inspira esta tese. Sua enorme cultura matemática e invejävel biblioteca foram imprescindiveis na elaboração das päginas que se seguem.

Ao Professor Doutor Antônio Gilioli, agradeço as inumeras sugestöes que me fêz ao ler a primeira redação do manuscrito deste trabatho.

Os meus agradecimentos especiais ao Professor. Doutor Lêo Roberto Borges Vieira pela paciência com que me ouviu e pelas suas sugestöes.

Aos calegas Amadeu Peter Hiller, Carmen Silvia Cardassi, Dra. Eeza Furtado Gomide, Moria Ignez de Souza Vieira Diniz e Zara Issa Abud, por me obrigarem a teminar este trabalho.

A minha esposa, pela colaboração direta e indireta, meu muito obrigado.

Finalmente, ao sr. Joño Baptista Esteves de Oliveira, pelo excelente trabalho de datilografia, minha gratidão. 



\section{ABSTRACT}

$X$ and $Y$ denote Banach spaces. We give partial answers to the following quostions:

1. Caracterize the Banach spaces $Y$ for which the elements of SV $(\{a, b \mid, l,(X, Y))$ and $R([a, b], Y)$ are strongly measurable.

2. Caracterize the Banach spaces $Y$ for which $D([a, b], Y)=$ $=R([a, b], Y)$.

Our main results are:

1) If, $X \neq\{0\}, \operatorname{SV}([a, b], L(X, Y)) \subseteq G([a, b], L(X, Y))$ if and only if $Y$ has no subspace isomorphic to $C_{0}(\mathbb{N})$ (Theorem 3.a.2).

2) There are infinite dimensional Banach spaces (even reflexive ones) such that $D([a, b], Y)=R([a, b], Y)$ (theorems 3.b.12, 3.b.13,3.b.14 and 3.b.20).

We also solve other related questions such as:

3) $\operatorname{SV}([a, b], L(X, Y)) \subseteq G([a, b], L(X, Y))$ does not imply that $Y$ is weckly sequentially complete.

4) Let fESV $([a, b], L(X, Y))$ and $t_{0} \in[a, b[$. The existence of 


$$
\lim _{t \rightarrow t_{0}} f(t)=f\left(t_{0}^{+}\right)
$$

does not imply that

$$
\left.\lim _{\substack{\varepsilon \rightarrow 0 \\ \varepsilon>0}} S V_{0}, t_{0}+\varepsilon\right][f]=0 .
$$

5) Classification, regarding problem 2 above, of important categories of Banach spaces such as uniformly convex, spaces $L_{p}(u), C(K)$ ( $K$ a compact Hausdorff space), etc.

6) We caracterize the Banach spaces $Y$ which are duals and for which every element of $\operatorname{BW}([a, b], Y)$ is strongly measurable.

7) There are compact Hausdorfe spaces $K$ such that $C(K)$ is not separable and every element of $B W([a, b], C(K))$, is strongly measurable. 


\section{INTRODUÇĀO}

o objetivo deste trabalho ê resolver questões que supomos em aberto na teoria de integração, segundo Riemann, de funções definidas num intervalo $[a, b]$ a valores num espaço de Banach.

No capítulo 1 estudamos as noçöes básicas de integração de que necessitamos e propomos os problemas a serem resolvidos em 1 . e.1. Tais problemas säo:

[P-1] - Caxactexizar os espaços de Banach X. para os quais $\operatorname{BV}([a, b], X) \notin B W([a, b], X)$.

[P-2] - Dar uma condição necessäria e suficiente sobre um espaço de Banach $X$ para que BW $([a, b], X) \subset G([a, b], X)$.

$[P-3]$ - Se $x$ è um espaço de Banach de dimensão infinita, podemos afirmar que $D([a, b], X) \notin R([a, b], X)$ ? (Este problema está proposto em [H-2] como problema 1.1).

[P-4] - Seja X um espaço de Banach. Dar uma condiçãonecessấria e suficiente sobre $X$ para que $f:[a, b] \rightarrow X$ seja Riemann integrâve 1 . 
[P-5] - Caracterizar os espaços de Banach Y para os quais $\operatorname{SV}([a, b], L(X, Y)) \subset G([a, b], L(X, Y))$. (Este problema é uma generalização do problema [P-2]).

$[P-6]-\operatorname{Se} \operatorname{SV}([a, b], L(X, Y)) \subset G([a, b], L(X, Y))$ temos necessariamente Y francamente sequencialmente completo? (Veja [H-5], página 13, problema 5).

$[P-7]$ - Seja $\operatorname{fESV}([a, b], L(X, Y))$ e $t_{0} \in[a, b[$ tal que exis-

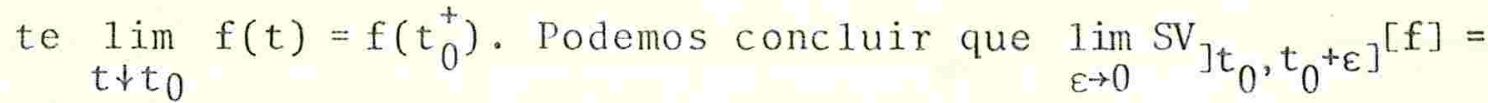
$=0$ ? (Veja [H-2], pägina 185, problema 7.4). $\varepsilon>0$

[P-8] - Dar um exemplo de $\operatorname{fECSV}([a, b], L(X))$ e deD ta1 que $\operatorname{SV}_{d}[f]-\left\|f\left(t_{1}\right)-f(a)\right\|>S V[f]-S_{\left[a, t_{1}\right]}[f] .(V e j a[H-2]$, pägina 186, problema 7.5 ).

Ainda neste capitulo 1 resolvemos o problema [P-1]. No capítulo 2 apresentamos um resumo dos elementos de geometria dos espaços de Banach, necessärios para as so1 uções dos problemas propostos no capítulo 1.

No capítulo 3 resolvemos, em 3 .a, os problemas [P-2], $[\mathrm{P}-5],[\mathrm{P}-6],[\mathrm{P}-7]$ e $[\mathrm{P}-8]$. Corrigimos duas afirmações incorretas na obra de nosso orientador:

$[E-1]-\operatorname{Se} f \in B W([a, b], X)$ e existe $f\left(t_{0}^{+}\right), t_{0} \in[a, b[$ então $\left.\left.\lim _{\varepsilon \rightarrow 0} B W_{0}\right] t_{0}, t_{0}+\varepsilon\right][f]=0$. (Veja $[H-2]$, pägina 185 , duas 1 inhas $\varepsilon>0$ finais).

[E-2] - Teorema 4.1 de [H-1], pägina 48 .

Para terminar o parágrafo $3 . a$, propomos dois pro- 
blemas sobre mensurabilidade de funções de $B W([a, b], x)$ e enunciamos os resultados obtidos. Tais resultados sãosurpreendentes pois, em certas classes de espaços de Banach, mos.tram "porque" existem funçöes de $B W([a, b] ; x)$ (portanto, integräveis Riemann! que sio náo mensuráveis.

No item $3 . b$ resolvemos o problema [P-3], mostrando a existência de espaços de Banach de dimensão infinita (e mesmo reflexivos) tais que $D([a, b], X)=R([a, b], X)$. Classificamos ainda categorias importantes de espaços de Banach quanto ao problema $[\mathrm{P}-3]$, tais como: Espaços uniformemente convexos, $C(K)$ ( $K$ compacto, Hausdorff), $[C(K, \mathbb{R})] ; L_{p}(u)$, etc.

Para terminar, em 3.c damos aplicaçöes dos resultados obtidos em 3.b, resolvendo problemas propostos em seminärios por nosso orientador.

Gostariamos de comentar que o problema $[\mathrm{P}-4]$ continua em aberto, bem como seu caso particular: "Caracterizar os espaços de Banach $x$ para os quais temos $D([a, b], X)=$ $=R([a, b], X) "$. Esta ültima questão lembra a situação do problema de Radon-Nikodym (veja [C]), que se revelou ligado à geometria dos espaços de Banach e que deu lugar a uma lite-ratura abundante e a uma fërtil ärea de pesquisa, como se pode ver pelo livro de Dieste1,J. \& Uh] Jr.,J.J., Vecton Measures, Providence, AMS, 1977, 322 p. (Ma thematical Surveys, 15).

Questões que supomos ainda com maior grau de dificuldade são as propostas em [0-1] e [Q-2], em 3.a, que, resumidamente, sẵo: "Porque em um espaço de Banach $x$ existem 
funções pertencentes aos espaços $B W([a, b], x)$ e $R([a, b], x)$ (portanto, integrảveis Riemann) não mensurảveis?". 


\section{CAPITULO 1}

NOÇŌES SOBRE A INTEGRAL DE RIEMANN EM ESPAÇOS DE BANACH

\section{1.a - NOÇŌES SOBRE INTEGRABILIDADE SEGUNDO DARBOUX \\ E INTEGRABILIDADE SEGUNDO RIEMANN}

$\underline{\text { DEFINIÇĀO }} \underline{1 . a .1}$ - Dado o intervalo [a,b], subconjunto de $\mathbb{R}$, denotaremos por d uma divisão deste intervalo, isto é, $\mathrm{d}=$ $=\left\{a=t_{0}<t_{1}<\ldots<t_{n}=b\right\}$. Denotaremos $\Delta d=\max _{1 \leq i \leq n}\left\{t_{i}-t_{i-1}\right\}$ e $|d|=$ $=n$. D indica o conjunto de todas as divisões de [a,b]. Se $\mathrm{d}_{1}$ e $\mathrm{d}_{2}$ são elementos de $D$, escreveremos $\mathrm{d}_{1} \geq \mathrm{d}_{2}$ para indicar que $\mathrm{d}_{2} \subseteq \mathrm{d}_{1}$.

$\underline{\text { DEFINICĀO }} \underline{1 . a .2}$ - Indicamos por $\Delta$ o conjunto de todas as sequências finitas $\delta=\left\{s_{1}, t_{1}, s_{2}, t_{2}, \ldots, s_{n}, t_{n}\right\} \operatorname{com} a s_{1}<t_{1} \leq$ $\leq s_{2}<t_{2} \leq \ldots \leq s_{n}<t_{n} \leq b$.

$\underline{\text { NOTAÇŌES }} \underline{1 . a .3}$ - Seja X um espaço de Banach. Utilizaremos as seguintes notações:

$C([a, b], X)$ - Conjunto das funções $f:[a, b] \rightarrow X$ contínuas. $B([a, b], X)$ - Conjunto das funções $f:[a, b] \rightarrow X$ limitadas. $L(X, Y)$ - Conjunto das aplicações 1 ineares contínuas de $\mathrm{X}$ em 
Y. (Y espaço de Banach).

$\omega_{i}(f)$ - Oscilação da função f no intervalo $\left[t_{i-1}, t_{i}\right]$, cujos extremos pertencem a una divisão d de $[a, b]$.

Dados $X$ e $Z$, espaços de Banach, $C^{b}(X, Z)$ indica $o$ conjunto das funções $\mathrm{f}: \mathrm{X} \longrightarrow \mathrm{Z}$ contínuas e que levam conjuntos Iimitados de $X$ em conjuntos limitados de $Z$.

$E([a, b], X)-$ Conjunto das funções $f:[a, b] \longrightarrow X$ em escada, isto $\vec{e}$, existe deD e $c_{1}, c_{2}, \ldots, c_{|d|} \in X$, tais que $f(x)=c_{i}$ para $x \in] t_{i-1}, t_{i}\lceil$.

$\underline{\text { DEFINICĀO }} \underline{1 . a \cdot 4}$ - Seja X um espaço de Banach e $f:[a, b] \rightarrow X$. Diremos que $f \bar{e}$ integrävel segundo Riemann, e escrevemos

$$
f \in R([a, b], x) \text {, se existe } \int_{a}^{b} f(x) d x=\lim _{\Delta d \rightarrow 0} \sum_{i=1}^{|d|} f\left(\varepsilon_{i}\right)\left[t_{i}-t_{i-1}\right]
$$

$\operatorname{com} \varepsilon_{i} \in\left[t_{i-1}, t_{i}\right]$.

PROPOSIÇĀO 1.a.5 (Critério de Cauchy) - Dado X um espaço de Banach e $f:[a, b] \rightarrow x$, são equivalentes:

1) Existe $\int_{a}^{b} f(x) d x$.

2) Para todo $\varepsilon>0$, existe $\delta>0$ tal que, se $d_{1}$ e $d_{2}$ são divisões de $[\mathrm{a}, \mathrm{b}] \operatorname{com} \Delta \mathrm{d}_{1} \leq \delta$ e $\Delta \mathrm{d}_{2} \leq \delta$ então

$$
\left\|\sum_{i=1}^{\left|d_{1}\right|} f\left(\varepsilon_{i}\right)\left[t_{i}-t_{i-1}\right]-\sum_{i=1}^{\left|d_{2}\right|} f\left(\varepsilon_{i}^{\prime}\right)\left[t_{i}-t_{i-1}\right]\right\| \leq \varepsilon,
$$

para toda escolha de $\varepsilon_{i} \in\left[t_{i-1}, t_{i}\right]$ e $\varepsilon_{i}^{\prime} \in\left[t_{i-1}^{\prime}, t_{i}^{\prime}\right]$.

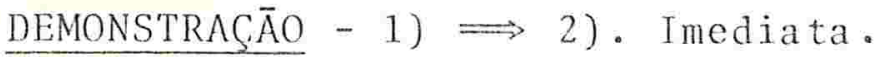

2) $\Longrightarrow 1$ ). Basta lembrar que o espaço X é completo. CQD 
COROLĀRIO 1.a.6 (Critērio de Cauchy modificado). Dado X espaço de Banach e $f:[a, b] \longrightarrow x$, são equivalentes:

1) $\operatorname{f\in R}([a, b], x)$.

2) Para todo $\varepsilon>0$, existe $\delta>0$ ta 1 que, para quaisquer $\overrightarrow{\mathrm{d}}, \mathrm{d} \in \mathcal{D}, \overline{\mathrm{d}} \geq \mathrm{d}$ e $\Delta \mathrm{d} \leq \delta$, temos que

$$
\left\|\sum_{i=1}^{|\bar{d}|} f\left(\varepsilon_{i}\right)\left[t_{i}-t_{i-1}\right]-\sum_{i=1}^{|d|} f\left(\varepsilon_{i}^{\prime}\right)\left[t_{i}-t_{i-1}\right]\right\| \leq \varepsilon .
$$

DEMONSTRAÇĀO -1$) \Longrightarrow 2$ ). E consequência da proposição 1.a.5: lembrando que $\overline{\mathrm{d}} \geq \mathrm{d} \Longrightarrow \Delta \overline{\mathrm{d}} \leq \Delta \mathrm{d} \leq \delta$, vem que :

$$
\left\|\sum_{i=1}^{|\vec{d}|} f\left(\varepsilon_{i}\right)\left[t_{i}-t_{i-1}^{1}\right]-\sum_{i=1}^{|d|} f\left(\varepsilon_{i}^{\prime}\right)\left[t_{i}-t_{i-1}\right]\right\| \leq \varepsilon .
$$

2) $\Longrightarrow 1)$. Dado $\varepsilon>0$, seja $\frac{\varepsilon}{2}>0$. Consideremos $\delta>0$ dado por 2) e divisões $d_{1}$ e $d_{2}$ tais que $\Delta d_{1} \leq \delta$ e $\Delta d_{2} \leq \delta$. Se $d_{=}=d_{1} u d_{2}$, virä:

$$
\begin{aligned}
& \left|\mathrm{d}_{1}\right| \quad\left|\mathrm{d}_{2}\right| \\
& \left\|\sum_{i=1} f\left(\varepsilon_{i}\right)\left[t_{i}-t_{i-1}\right]-\sum_{i=1} f\left(\varepsilon_{i}^{\prime \prime}\right)\left[t_{i}^{\prime \prime}-t_{i-1}^{\prime \prime}\right]\right\| \leq \\
& \left|\mathrm{d}_{1}\right| \\
& \leq\left\|\sum_{i=1}^{1} f\left(\varepsilon_{i}\right)\left[t_{i}-t_{i-1}\right]-\sum_{i=1}^{|d|} f\left(\varepsilon_{i}^{\prime}\right)\left[t_{i}-t_{i-1}^{\prime}\right]\right\|+ \\
& +\left\|\sum_{i=1}^{|d|} f\left(\varepsilon_{1}^{\prime}\right)\left[t_{i}^{\prime}-t_{i-1}^{\prime}\right]-\sum_{i=1}^{\left|d_{2}\right|} f\left(\varepsilon_{i}^{\prime \prime}\right)\left[t_{i}^{\prime \prime}-t_{i-1}^{\prime \prime}\right]\right\| \leq \frac{\varepsilon}{2}+\frac{\varepsilon}{2}=\varepsilon \text {. }
\end{aligned}
$$

Pela proposição $1 . a .5,2) \Longrightarrow 1)$ c existe $\int_{a}^{b} f(x) d x . \quad C Q D$.

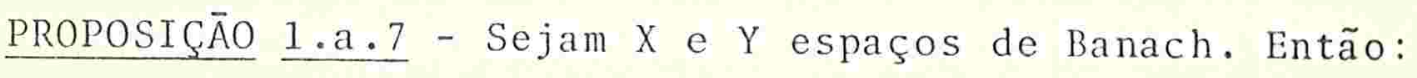

1) $R([a, b], X) \subset B([a, b], X)$. 
2) Se $f \in R([a, b], X)$ e $u \in L(X, Y)$, temos:

$$
u \circ f \operatorname{ER}([a, b], y) \text { e } \int_{a}^{b}(u \circ f)(x) d x=u\left[\int_{a}^{b} f(x) d x\right] \text {. }
$$

3) Se $J: X \rightarrow Y$ é um isomorfismo linear, são equivalentes: a) $f \in R([a, b], X) ; b) \operatorname{Jof\in R}([a, b], Y)$.

4) $R([a, b], x)$ só depende da topologia de $x$.

5) Se $\left\{x_{i}\right\}_{i=1}^{n}$ são espaços de Banach, $x=\prod_{i=1}^{n} X_{i}$ e $P_{i}: X_{\rightarrow} \rightarrow x_{i}$, $1 \leq \mathrm{i} \leq \mathrm{n} \overrightarrow{\mathrm{e}}$ tal que, se $\mathrm{x}=\left(\mathrm{x}_{1}, \mathrm{x}_{2}, \ldots, \mathrm{x}_{\mathrm{n}}\right)$ então $\mathrm{P}_{i}(\mathrm{x})=\mathrm{x}_{i}$, $1 \leq i \leq n$, são equivalentes a) e b) abaixo:

a) $\operatorname{f\in R}([a, b], x)$, b) $f_{i}=P_{i} \circ f \in R\left([a, b], x_{i}\right), 1 \leq i \leq n$.

Temos ainda

$$
\int_{a}^{b} f(x) d x=\left\{\int_{a}^{b} f_{i}(x) d x\right\}_{i=1}^{n}
$$

DEMONSTRAC, $\bar{A} O-1)$ Se $f \notin B([a, b], x)$, encontramos $\left\{x_{n}\right\}_{n=1}^{\infty} c[a, b]$ tal que $\left\|f\left(x_{n}\right)\right\| \stackrel{n \rightarrow \infty}{\longrightarrow} \infty$. Seja $x_{0} \in[a, b]$ ponto de acumulação de $\left\{x_{n}\right\}_{n=1}^{\infty}$

Dado $\delta>0$, tomemos uma divisão deO com $\Delta \mathrm{d} \leq \delta$, tal que $x_{0} \in\left[t_{i_{0}-1}, t_{i_{0}}\right]$ e $\left\{x_{n}\right\}_{i=1}^{\infty} n\left[t_{i_{0}-1}, t_{i_{0}}\right]$ é um conjunto infinito.

Vale que

$$
\begin{aligned}
& \left\|\sum_{i=1}^{|d|} f\left(\varepsilon_{i}\right)\left[t_{i}-t_{i-1}\right]-\int_{a}^{b} f(x) d x\right\|= \\
= & \left\|f\left(\varepsilon_{i_{0}}\right)\left[t_{i_{0}}-t_{i_{0}-1}\right]-\left[\int_{a}^{b} f(x) d x-\sum_{\substack{i=1 \\
i \neq i_{0}}}^{|d|} f\left(\varepsilon_{i}\right)\left[t_{i}-t_{i-1}\right]\right]\right\| .
\end{aligned}
$$


Se $\varepsilon=1$, fixando $\varepsilon_{i} \in\left[t_{i-1}, t_{i}\right]$, para todo $i \neq i_{0}$, vem:

$$
\left\|f\left(\varepsilon_{i_{0}}\right)\left[t_{i_{0}}-t_{i_{0}-1}\right]\right\| \leq\left\|\int_{a}^{b} f(x) d x-\sum_{\substack{i=1 \\ i \neq i_{0}}}^{|d|} f\left(\varepsilon_{i}\right)\left[t_{i}-t_{i-1}\right]\right\|+1=M+1
$$

Se fizermos $\varepsilon_{i_{0}}$ tender a $x_{0}$ por pontos de $\left\{x_{n}\right\}_{n=1}^{\infty}$ virá $M=\infty$ : absurdo .

2) Se $u=0$, a afirmação é evidente. Se $u \neq 0$, dado $\frac{\varepsilon}{\|u\|}>0$ tomemos $\delta>0$ tal que, se

$$
\Delta d \leq \delta, \quad\left\|\sum_{i=1}^{|d|} f\left(\varepsilon_{i}\right)\left\lceil t_{i}-t_{i-1}\right]-\int_{a}^{b} f(x) d x\right\| \leq \frac{\varepsilon}{\|u\|} .
$$

Então, se

$$
\begin{aligned}
\Delta d & \leq \delta,\left\|\sum_{i=1}^{|d|}(u \circ f)\left(\varepsilon_{i}\right)\left[t_{i}-t_{i-1}\right]-u\left(\int_{a}^{b} f(x) d x\right)\right\| \leq \\
& \leq\|u\|\left\|\sum_{i=1}^{|d|} f\left(\varepsilon_{i}\right)\left[t_{i}-t_{i-1}\right]-\int_{a}^{b} f(x) d x\right\| \leq \varepsilon ;
\end{aligned}
$$

logo,

$$
u \circ f \in R([a, b], Y) \text { e } \int_{a}^{b}(u \circ f)(x) d x=u\left(\int_{a}^{b} f(x) d x\right) .
$$

3) Consequência imediata de 2).

4) Consequência imediata de 3).

5) a) $\Longrightarrow$ b). Decorrência imediata de 2), pois $P_{i}, 1 \leq i \leq n$, são funções lineares e contínuas.

b) $\Longrightarrow$ a). Tomemos em $x=\prod_{i=1}^{n} x_{i},\|x\|=\sup _{1 \leq i \leq n}\left\|x_{i}\right\|_{i}$. 
Dado $\varepsilon>0$, consideremos $\delta>0$ tal que, se $d \in D$ e $\Delta d \leq \delta$,

$$
\sup _{1 \leq i \leq n}\left\|\sum_{i=1}^{|d|} f_{i}\left(\varepsilon_{i}\right)\left[t_{i}-t_{i-1}\right]-\int_{a}^{b} f_{i}(x) d x\right\| \leq \varepsilon .
$$

Então,

$$
\begin{aligned}
& \left\|\sum_{i=1}^{|d|} f\left(\varepsilon_{i}\right)\left[t_{i}-t_{i-1}\right]-\left\{\int_{a}^{b} f_{i}(x) d x\right\}_{i=1}^{n}\right\|= \\
= & \sup _{1 \leq i \leq n}\left\|\sum_{i=1}^{|d|} f_{i}\left(\varepsilon_{i}\right)\left[t_{i}-t_{i-1}\right]-\int_{a}^{b} f_{i}(x) d x\right\| \leq \varepsilon,
\end{aligned}
$$

o que demonstra que

$$
f \in R([a, b], x) \text { e } \int_{a}^{b} f(x) d x=\left\{\int_{a}^{b} f_{i}(x) d x\right\}_{i=1}^{n} \cdot C Q D
$$

$\underline{\text { DEFINIÇĀO }} \underline{1 . \mathrm{a} .8}$ - Seja X um espaço de Banach e $\mathrm{f}:[\mathrm{a}, \mathrm{b}] \rightarrow \mathrm{X}_{\text {。 }}$ Diremos que $f \bar{e}$ integrável segundo Darboux, e escrevemos

$$
f \in D([a, b], x), \text { se } \lim _{\Delta d \rightarrow 0} \sum_{i=1}^{|d|} w_{i}(f)\left[t_{i}-t_{i-1}\right]=0 \text {. }
$$

$\underline{\text { PROPOSIÇÃO }} \underline{1 . a .9}$ - Dados X e Y espaços de Banach, temos:

1) $D([a, b], X) \subset R([a, b], X) \subset B([a, b], X)$. (Então passa a ter sentido $\int_{a}^{b} f(x) d x$, para toda $\left.f \in D([a, b], X)\right)$.

2) Se $f \in D([a, b], X)$ e $u \in L(X, Y)$ então $u \circ f \in D([a, b], Y)$.

3) Se $J: X \rightarrow Y$ é um isomorfismo linear, são equivalentes a) e b) abaixo:

a) $f \in D([a, b], X) ; b) \quad J \circ f \in D([a, b], Y)$. 
4) $D([a, b], x)$ só depende da topologia de $x$.

5) Se $\left\{x_{i}\right\}_{i=1}^{n}$ são espaços de Banach, $x=\prod_{i=1}^{n} x_{i}$ e $P_{i}: x \rightarrow x_{i}$, $1 \leq i \leq n$, è tal que, se $x=\left(x_{1}, x_{2}, \ldots, x_{n}\right), P_{i}(x)=x_{i}, 1 \leq i \leq n$, são equivalentes a) e b) abaixo:
a) $f \in D([a, b], x)$;
b) $f_{i}=P_{i} \circ f \in D\left([a, b], x_{i}\right), 1 \leq i \leq n$.

Temos ainda

$$
\int_{a}^{b} f(x) d x=\left\{\int_{a}^{b} f_{i}(x) d x\right\}_{i=1}^{n}
$$

DEMONSTRAÇĀO - 1) Vamos usar a proposição 1. a.6 nesta demonstração.

Se $f \in D([a, b])$, dado $\varepsilon>0$, consideremos $\delta>0$ tal que, se $\Delta d \leq \delta$ então

$$
\sum_{i=1}^{\mid d} \omega_{i}(f)\left[t_{i}-t_{i-1}\right] \leq \varepsilon \text {. }
$$

Consideremos $\overline{\mathrm{d}} \geq \mathrm{d}$ e teremos, efetuando um cálculo simp1es :

$$
\left\|\sum_{i=1}^{|\bar{d}|} f\left(\varepsilon_{i}\right)\left[t_{i}^{\prime}-t_{i-1}^{\prime}\right]-\sum_{i=1}^{|d|} f\left(\varepsilon_{i}^{\prime}\right)\left[t_{i}-t_{i-1}\right]\right\| \leq \sum_{i=1}^{|d|} \omega_{i}(f)\left[t_{i}-t_{i-1}\right] \leq \varepsilon .
$$

Pela proposição 1.a.6, $\mathrm{f} \in \mathrm{R}([\mathrm{a}, \mathrm{b}], \mathrm{X})$. Portanto, $D([a, b], x) \subset R([a, b], x)$.

A segunda inclusão foi demonstrada na proposição 1.a.7, item 1 .

2) Se $u=0$ o teorema ê imediato. Se $u \neq 0$, observemos que 


$$
\omega_{i}[u \circ f]=\sup _{\varepsilon_{i}, \varepsilon_{i}^{\prime} \in\left[t_{i-1}, t_{i}\right]}\left\|(u \circ f)\left(\varepsilon_{i}\right)-(u \circ f)\left(\varepsilon_{i}^{\prime}\right)\right\| \leq\|u\| \omega_{i}[f] .
$$

Portanto, dado $\varepsilon>0$, existe $\delta>0$, se d $\in D$, com $\Delta d \leq \delta$, virä

$$
\sum_{i=1}^{|d|} \omega_{i}[f]\left[t_{i}-t_{i-1}\right] \leq \frac{\varepsilon}{\|u\|}
$$

Então, se $\Delta d \leq \delta$ temos:

$$
\sum_{i=1}^{|d|} \omega_{i}[u \circ f]\left[t_{i}-t_{i-1}\right] \leq\|u\| \sum_{i=1}^{|d|} \omega_{i}[f]\left[t_{i}-t_{i-1}\right] \leq \varepsilon_{i} .
$$

$\log \mathrm{u} u f \in D([a, b], Y)$.

3) Decorrência imediata de 2).

4) Decorrência imediata de 3).

5) a) $\Longrightarrow$ b). Decorrência imediata de 2).

b) $\Longrightarrow$ a). Consideremos em $x=\prod_{i=1}^{n} x_{i},\|x\|=\sup _{1 \leq i \leq n}\left\|x_{i}\right\|_{i}$. Então, um cảlculo simples mostra que $\omega_{i}[f]=\sup _{1 \leq j \leq n} \omega_{i}\left[f_{j}\right]$. Portanto, se tomarmos $\frac{\varepsilon}{n}>0$ existe $\delta>0$ tal que, se d $\in D$, com

$$
\Delta d \leq \delta, \quad \sum_{i=1}^{|d|} \omega_{i}\left[f_{i}\right]\left[t_{i}-t_{i-1}\right] \leq \frac{\varepsilon}{n}, \quad 1 \leq j \leq n,
$$

e virä que

$$
\begin{aligned}
\sum_{i=1}^{|d|} \omega_{i}[f]\left[t_{i}-t_{i-1}\right] & =\sum_{i=1}^{|d|}\left(\sup _{1 \leq j \leq n} \omega_{i}\left[f_{j}\right]\right)\left[t_{i}-t_{i-1}\right] \leq \\
& \leq \sum_{j=1}^{n}\left(\sum_{i=1}^{|d|} \omega_{i}\left[f_{j}\right]\left[t_{i}-t_{i-1}\right]\right) \leq n \frac{\varepsilon}{n}=\varepsilon ;
\end{aligned}
$$

logo, $f \in D([a, b], x)$. A üItima parte é decorrência imediata de 
1.a.7, item 5 .

CQD

$\underline{\text { PROPOSIÇĀO }} \underline{1 . a \cdot 10}$ - Seja X um espaço de Banach. Temos:

1) $\mathcal{C}([a, b], x) \subset D([a, b], x)$.

2) $\operatorname{Se} f \in D([a, b], X)$ e $g \in C^{b}(X, Z)$ então $g \circ f \in D([a, b], Z)$.

3) Se $f \in D([a, b], X)$ então $\|f\| \in D([a, b], R)$.

4) Sejam X, Y e $Z$ espaços de Banach, B: $X \times Y \rightarrow Z$ uma aplicação bilinear contínua, $f \in D([a, b], X)$ e $g \in D([a, b], Y)$.Escrevemos $(f \cdot g)(t)=f(t) \cdot g(t)=B(f(t), g(t))$. Então

$$
f \cdot g \in D([a, b], z) \text {. }
$$

5) Se A è uma ảlgebra de Banach então $D([a, b], A)$ è uma älgebra de Banach.

6) Se $f_{n} \in D([a, b], X)$ e $f_{n}$ converge uniformemente para $f$, então

$$
f \in D([a, b], x) \text { e } \int_{a}^{b} f_{n}(t) d t \stackrel{n \rightarrow \infty}{\longrightarrow} \int_{a}^{b} f(t) d t
$$

7) Se $f \in D([a, b], X)$ e $F:[a, b] \rightarrow X$ é dada por

$$
F(t)=\int_{a}^{t} f(x) d x
$$

então $\mathrm{F}$ é deriváve1 quase sempre.

8) São equivalentes as afirmações a) e b) abaixo:

a) $f \in D([a, b], X)$.

b) Para todo $\varepsilon>0$, existe $f_{\varepsilon} \in E([a, b], X)$ ta 1 que

$$
\overline{\int_{a}^{b}}\left\|f(t)-f_{\varepsilon}(t)\right\| d t \leq \varepsilon
$$


DEMONSTRAÇĀO - A demonstração do item 8 encontra-se em [H-1], página 62, B-9. As demonstrações restantes serão feitas no corolärio $1 . b \cdot 14$.

PROPOSIC,ĀO $1 . a \cdot 11-1) D([a, b], \mathbb{K})=R([a, b], \mathbb{K}), s c \mathbb{K}=\mathbb{R}$ ou $\mathbb{C}$.

2) Se $x$ é um espaço de Banach de dimensão finita então $D([a, b], x)=R([a, b], x)$.

DEMONSTRAÇĀO - 1) Faremos a demonstração para o caso rea1, já que o caso complexo é consequência imediata do caso real. Se $f \in R([a, b], \mathbb{R})$, vale a proposição $1 . a .5$ (Cauchy): dado $\varepsilon>0$, existe $\delta>0$ tal que, para $d \in D$, se $\Delta d<\delta$, então

$$
\left|\sum_{i=1}^{|d|}\left[f\left(\varepsilon_{i}\right)-f\left(\varepsilon_{i}^{\prime}\right)\right]\left(t_{i}-t_{i-1}\right)\right| \leq \varepsilon,
$$

para quaisquer $\varepsilon_{i}, \varepsilon_{i}^{\prime} \in\left[t_{i-1}, t_{i}\right]$.

Em particular, escolhendo $\varepsilon_{i}, \varepsilon_{i}^{\prime}$ tais que $f\left(\varepsilon_{i}\right) \geq f\left(\varepsilon_{i}^{\prime}\right)$, para todo i, obtemos

$$
\sum_{i=1}^{|d|}\left|f\left(\varepsilon_{i}\right)-f\left(\varepsilon_{i}^{\prime}\right)\right|\left(t_{i}-t_{i-1}\right) \leq \varepsilon
$$

e vem que

$$
\sum_{i=1}^{|d|} \sup _{i, \varepsilon_{i}^{\prime}}\left|f\left(\varepsilon_{i}\right)-f\left(\varepsilon_{i}^{\prime}\right)\right|\left(t_{i}-t_{i-1}\right) \leq \varepsilon,
$$

ou seja,

$$
\sum_{i=1}^{|d|} \omega_{i}(f)\left(t_{i}-t_{i-1}\right) \leq \varepsilon
$$


$\operatorname{Logo}, \quad f \in D([a, b], \mathbb{R}), \quad e R([a, b], \mathbb{R}) \subset D([a, b], \mathbb{R})$.

Da proposição 1.a.9, item 1, concluímos:

$$
D([a, b], \mathbb{R})=R([a, b], \mathbb{R}) .
$$

Para o caso complexo, é imediato que, se $f=f_{1}+i f_{2}$, $f \in D([a, b], \mathbb{C}) \Longleftrightarrow f_{i} \in D([a, b], \mathbb{R}), i=1,2 \Longleftrightarrow f_{i} \in R([a, b], \mathbb{R}), i=1,2 \Longleftrightarrow$

$$
\Longleftrightarrow f=f_{1}+i f_{2} \operatorname{ER}([a, b], \mathbb{C})
$$

2) Se $X$ é um espaço de Banach de dimensão finita então $\mathrm{X} \overrightarrow{\mathrm{e}}$ isomorfo a $\mathbb{K}^{\mathrm{n}}:$ seja $\mathrm{J}: \mathrm{X} \longrightarrow \mathbb{K}^{\mathrm{n}}$ este isomorfismo.Nestas condições,

$$
\begin{aligned}
& f \in R([a, b], x) \stackrel{1 . a \cdot 7.3)}{\Longleftrightarrow} \operatorname{Jof\in R}\left([a, b], \mathbb{K}^{\mathrm{n}}\right) \stackrel{1 . a \cdot 7.5)}{\Longleftrightarrow} \\
& \stackrel{1 . a .7 .5)}{\Longrightarrow} P_{i} \circ J \circ f \in R([a, b], \mathbb{K}) \stackrel{1 . a \cdot 11.1)}{=} D([a, b], I K) \stackrel{1 . a .9 .5)}{\rightleftharpoons} \\
& \stackrel{1 . a .9 .5)}{\Longleftrightarrow} \operatorname{Jof} \in D\left([a, b], \mathbb{K}^{n}\right) \stackrel{1 . a .9 .3)}{\Longleftrightarrow} \mathrm{fED}([\mathrm{a}, \mathrm{b}], \mathrm{x}) \cdot \quad \text { CQD }
\end{aligned}
$$

\section{1.b- FUNÇÖES DE INTERVALO E APLI CAÇŌES}

No que segue, farcmos um breve resumo sobre funções de intervalo, de onde tiraremos consequências que nos serão üteis. Inspiramo-nos no livro texto: [H].

$\underline{\text { NOTAÇĀO }} 1 . \mathrm{b} \cdot 1$ - Seja $\mathrm{X}$ um espaço de Banach e $\mathrm{f}:[\mathrm{a}, \mathrm{b}] \rightarrow \mathrm{X}$. Utilizaremos as seguintes notações:

$$
\begin{aligned}
\omega(A, f)- & \text { oscilação da função } f \text { no conjunto } A, \text { não vazio } \\
& \text { e não unitário, contido em }[a, b] . \\
\omega(\{t\}, f)= & \left.\inf _{\delta>0}\{\omega(] t-\delta, t+\delta[, f)\} \text { se } t \in\right] a, b[
\end{aligned}
$$


$\omega(\{a\}, f)=\inf _{\delta>0}\left\{\omega\left([a, a+\delta[, f)\} ; \omega(\{b\}, f)=\inf _{\delta>0}\{\omega(] b-\delta, b], f\right)\right\}$.

$D(f)$ - Conjunto dos pontos de descontinuidade da função f.

u - Medida de Lebesgue em $\mathbb{R}$.

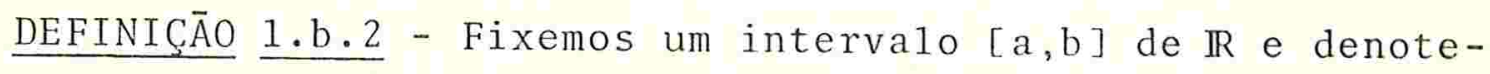
mos por $I=I_{[a, b]}$ o conjunto dos subintervalos $[c, d]$ de $[a, b]$. Dado um espaço de Banach $X$, se $P(X)$ é o conjunto de suas partes, denominaremos funções de intervalo a uma função

$$
\mathrm{F}: \mathrm{I} \longrightarrow P(X) \text {. }
$$

\section{$\underline{1 . b .3}-$ EXEMPLOS IMPORTANTES}

Seja $I=[c, d] c[a, b], X$ espaço de Banach e $f:[a, b] \rightarrow X$.

1) Considere $F: I \rightarrow P(X)$, por $F(I)=\left\{f\left(\varepsilon_{i}\right)[d-c] / \varepsilon_{i} \in[c, d]\right\}$.

2) Considere $F: I \longrightarrow P(\mathbb{R})$, dada por $F(I)=\omega(I, f)[d-c]$. $\underline{\text { DEFINIÇĀO }} \underline{1 . \mathrm{b} .4}$ - Dada F: I $\longrightarrow$ P(X) função de intervalo, seja $S_{F}(d): D \longrightarrow P(X)$, definida por $S_{F}(d)=\sum_{i=1}^{|d|} F\left(I_{i}\right)$, onde $I_{i}=\left[t_{i-1}, t_{i}\right]$.

Diremos que $F$ tem integral na norma em [a,b] (respectivamente $\sigma$-integral em $[a, b])$ se existe

$$
\lim _{\Delta d \rightarrow 0} S_{F}(d)=\lim _{\Delta d \rightarrow 0} \sum_{i=1}^{|d|} F\left(I_{i}\right)=N \int_{a}^{b} F
$$

(respectivamente,

$$
\left.\lim _{d \in D} S_{F}(d)=\lim _{d \in D} \sum_{i=1}^{|d|} F\left(I_{i}\right)=\sigma \int_{a}^{b} F\right)
$$


isto é, existe $N \int_{a}^{b} F \in X$ com a propriedade: para todo $\varepsilon>0$, existe $\delta>0$ tal que, se $d \in D$ e $\Delta d \leq \delta$ então $\left\|S_{F}(d)-N \int_{a}^{b} F\right\| \leq \varepsilon$, onde

$$
\left\|S_{F}(d)-N \int_{a}^{b} F\right\|=\sup \left\{\left\|x-N \int_{a}^{b} F\right\| / x \in S_{F}(d)\right\}
$$

(respectivamente, existe $\sigma \int_{a}^{b} F E X$ com a propriedade: para todo $\varepsilon>0$, existe $d_{\varepsilon}$ tal que se $d \in D$ e $d \geq d_{\varepsilon}$, então

$$
\left\|S_{F}(d)-\sigma \int_{a}^{b} F\right\| \leq \varepsilon
$$

onde

$$
\left\|S_{F}(d)-\sigma \int_{a}^{b} F\right\|=\sup \left\{\left\|x-\sigma \int_{a}^{b} F\right\| / x \in S_{F}(d)\right\} .
$$

Aplicados os dois limites acima aos itens 1 e 2 do exemplo 1.b.3, obtemos: no item 1, dois conceitos de Integrabilidade segundo Riemann. As integrais obtidas serão denotadas, respectivamente, por $N \int_{a}^{b} f(x) d x$ e $o \int_{a}^{b} f(x) d x$, sendo o primeiro conceito coincidente com o definido em 1.a.4; portanto $N \int_{a}^{b} f(x) d x=\int_{a}^{b} f(x) d x$. No item 2 se exigirmos $N \int_{a}^{b} F=0$ e $\sigma \int_{a}^{b} F=$ $=0$, temos dois conceitos de função integrảvel segundo Darboux, sendo o primeiro coincidente com o conceito definido em 1.a.8.

$\underline{\text { PROPOSIC̄ĀO } 1 . \mathrm{b} .5}$ - Seja F: I $\longrightarrow P(X)$ uma função de intervalo. Temos:

1) Se existe $N \int_{a}^{b} F$ então existe $\sigma \int_{a}^{b} F$ e $N \int_{a}^{b} F=\sigma \int_{a}^{b} F$.

2) Seja $x$ um espaço de Banach e $f:[a, b] \longrightarrow X$. 
Se existe $N \int_{a}^{b} f(x) d x=\int_{a}^{b} f(x) d x$ então existe

$$
\sigma \int_{a}^{b} f(x) d x \quad \text { e } \int_{a}^{b} f(x) d x=\sigma \int_{a}^{b} f(x) d x .
$$

DEMONSTRACSĀO - Imediata.

No que segue, toda vez que usarmos o conceito de norma envolvendo diferença de conjuntos A e B, o significado serä: $\|A-B\|=\sup _{\substack{a \in A \\ b \in B}} \mid a-b \|$.

$\underline{\text { PROPOSIÇĀO }} \underline{1 . b .6}$ - 1$)$ Se existe $\sigma \int_{a}^{b} f(x) d x$ então $f \in B([a, b], x)$.

2) Se $\lim _{d \in D} \sum_{i=1}^{|d|} \omega_{i}(f)\left[t_{i}-t_{i-1}\right]=0$ então $f \in B([a, b], x)$.

DEMONSTRAÇĀO - Anāloga à da proposição 1.a.7, item 1.

$\underline{\text { PROPOSIÇĀO }} \underline{1 . b .7}$ - Dada uma função de intervalo $F: I \rightarrow P(X)$, uma condição necessäria e suficiente para que exista $N \int_{a}^{b} F$ (respectivamente $\sigma \int_{a}^{b} F$ ) è que: para todo $\varepsilon>0$, exista $\delta>0$ tal que, se $\mathrm{d}_{1} \mathrm{~d}_{2} \in D, \Delta \mathrm{d}_{1} \leq \delta$ e $\Delta \mathrm{d}_{2} \leq \delta$, então

$$
\left\|\sum_{i=1}^{\left|d_{1}\right|} F\left(I_{i}\right)-\sum_{i=1}^{\left|d_{2}\right|} F\left(I_{i}^{\prime}\right)\right\| \leq \varepsilon
$$

(respectivamente, para todo $\varepsilon>0$, exista $d_{\varepsilon}$ tal que, se $d_{1} \geq$ $\geq \mathrm{d}_{\varepsilon}$ e $\mathrm{d}_{2} \geq \mathrm{d}_{\varepsilon}$ então

$$
\left.\left\|\sum_{i=1}^{\left|d_{1}\right|} F\left(I_{i}\right)-\sum_{i=1}^{\left|d_{2}\right|} F\left(I_{i}^{\prime}\right)\right\| \leq \varepsilon\right) .
$$

DEMONSTRAÇĀO - Imediata nas duas direções se lembrarmos que o espaço X è completo. 
$\underline{D E F I N I C \bar{A} O} \underline{1 . b .8}$ - Dada uma função de intervalo F:I $\rightarrow P(X)$, diremos que $F \vec{e}$ pseudo-aditiva no ponto $x \in] a, b[$ se

$$
\lim _{\left(y^{\prime}, y^{\prime \prime}\right) \rightarrow(0,0)}\left\{F\left(\left[x-y^{\prime}, x+y^{\prime \prime}\right]\right)-F\left(\left[x-y^{\prime}, x\right]\right)-F\left(\left[x, x+y^{\prime \prime}\right]\right)\right\}=0
$$

com $y^{\prime} \geq 0, \quad y^{\prime \prime} \geq 0$

OBSERVAÇĀO 1.b.9 - Se, nos exemplos 1.b.3, supusermos

$$
f:[a, b] \rightarrow x
$$

uma função integrävel no sentido das definições dadas em 1.b.4, obteremos exemplos de funções de intervalo pseudo-aditivas. De fato, no primeiro caso:

$$
\begin{aligned}
& F\left(\left[x-y^{\prime}, x+y^{\prime \prime}\right]\right)-F\left(\left[x-y^{\prime}, x\right]\right)-F\left(\left[x, x+y^{\prime \prime}\right]\right)= \\
& =f\left(\varepsilon_{i}\right)\left[y^{\prime \prime}+y^{\prime}\right]-f\left(\varepsilon_{i}^{\prime}\right)\left[y^{\prime}\right]-f\left(\varepsilon_{i}^{\prime \prime}\right)\left[y^{\prime \prime}\right],
\end{aligned}
$$

onde $\varepsilon_{i} \in\left[x-y^{\prime}, x+y^{\prime \prime}\right], \varepsilon_{i}^{\prime} \in\left[x-y^{\prime}, x\right], \varepsilon_{i}^{\prime \prime} \in\left[x, x+y^{\prime \prime}\right] ;$ como $f:[a, b] \rightarrow X$ è 1 imitada (proposição $1 . a .7$, item 1 e $1 . b .6$, item 1),

$$
\lim _{\left(y^{\prime}, y^{\prime \prime}\right) \rightarrow(0,0)}\left\{F\left[x-y^{\prime}, x+y^{\prime \prime}\right]-F\left[x-y^{\prime}, x\right]-F\left[x, x+y^{\prime \prime}\right]\right\}=0 .
$$

No segundo caso:

$$
\begin{aligned}
& F\left(\left[x-y^{\prime}, x+y^{\prime \prime}\right]\right)-F\left(\left[x-y^{\prime}, x\right]\right)-F\left(\left[x, x+y^{\prime \prime}\right]\right)=\left(y^{\prime \prime}-y^{\prime}\right) \cdot \\
& \cdot \omega\left(\left[x-y, x+y^{\prime \prime}\right], f\right)-y^{\prime} \omega\left(\left[x-y^{\prime}, x\right], f\right)-y^{\prime \prime} \cdot \omega\left(\left[x, x+y^{\prime \prime}\right], f\right) .
\end{aligned}
$$

Como f é 1imitada (proposição 1.a.9, item 1 e 1.b.6, item 2),

$$
\lim _{\left(y^{\prime}, y^{\prime \prime}\right) \rightarrow(0,0)}\left\{F\left(\left[x-y^{\prime}, x+y^{\prime \prime}\right]\right)-F\left(\left[x-y^{\prime}, x\right]\right)-F\left(\left[x, x+y^{\prime \prime}\right]\right)\right\}=0
$$


$\underline{\text { TEOREMA }} \underline{1 . \mathrm{b} .10}$ - Dada uma função de intervalo F:I $\longrightarrow P(X)$, são equivalentes:

1) Existe $N \int_{a}^{b} F$.

2) a) F é pseudo-aditiva em todo ponto de ]a,b[.

b) Existe $\sigma \int_{a}^{b} F$.

Nestas condições, $\sigma \int_{a}^{b} F=N \int_{a}^{b} F$.

\section{DEMONSTRAÇĀO -1$) \Longrightarrow 2$ )}

$1) \Longrightarrow 2 a)$ - Se existe $N \int_{a}^{b} F$ então, para $\varepsilon>0$, existe $\delta>0$ tal que, se $d_{1}, d_{2}$ são divisões de $[a, b] \operatorname{com} \Delta d_{1} \leq \delta$ e $\Delta d_{2} \leq \delta$ então

$$
\left\|\sum_{i=1}^{\left|d_{1}\right|} F\left(I_{i}\right)-\sum_{i=1}^{\left|d_{2}\right|} F\left(I_{i}^{\prime}\right)\right\| \leq \varepsilon
$$

Se $x \in] a, b\left[\right.$, tomemos $d_{1}$ com os pontos $x-y^{\prime}, x+y^{\prime \prime} e d_{2}=d_{1} u\{x\}$, cumprindo as condições $\Delta \mathrm{d}_{1} \leq \delta, \Delta \mathrm{d}_{2} \leq \delta$, isto é, $\mathrm{y}^{\prime} \leq \frac{\delta}{2}$ e $\mathrm{y}^{\prime \prime} \leq \frac{\delta}{2}$. Então

$\left\|\sum_{i=1}^{\left|d_{1}\right|} F\left(I_{i}\right)-\sum_{i=1}^{\left|d_{2}\right|} F\left(I_{i}^{\prime}\right)\right\|=\left\|F\left(\left[x-y^{\prime}, x+y^{\prime \prime}\right]\right)-F\left(\left[x-y^{\prime}, x\right]\right)-F\left(\left[x, x+y^{\prime \prime}\right]\right)\right\| \leq \varepsilon$.

$\log 0$

$$
\lim _{\left(y^{\prime}, y^{\prime \prime}\right) \rightarrow(0,0)}\left\{F\left(\left[x-y^{\prime}, x+y^{\prime \prime}\right]\right)-F\left(\left[x-y^{\prime}, x\right]\right)-F\left(\left[x, x+y^{\prime \prime}\right]\right)\right\}=0
$$

1) $\Longrightarrow 2 \mathrm{~b})-$ Se existe $N \int_{a}^{b} F$ então, para todo $\varepsilon>0$, existe $\delta>0$ ta1 que, se deD e $\Delta d \leq \delta$ então

$$
\left\|\sum_{i=1}^{|d|} F\left(I_{i}\right)-N \int_{a}^{b} F\right\| \leq \varepsilon
$$


Seja $d_{\varepsilon}$ uma divisão tal que $\Delta d_{\varepsilon} \leq \delta$. Se $d \geq d_{\varepsilon}$, virä que

$$
\left\|\sum_{i=1}^{|d|} F\left(T_{i}\right)-N \int_{a}^{b} F\right\| \leq \varepsilon \cdot \log \sigma \text {, existe } \sigma \int_{a}^{b} F \quad c \quad \sigma \int_{a}^{b} F=N \int_{a}^{b} F \text {. }
$$

2) $\Longrightarrow 1)$ - Como existe $\sigma \int_{a}^{b} F$ então, dado $\varepsilon>0$ existe $d_{\varepsilon}$ tal que, se $d \geq d_{\varepsilon}$, tem-se

$$
\left\|\sum_{i=1}^{|d|} F\left(I_{i}\right)-\sigma \int_{a}^{b} F\right\| \leq \varepsilon .
$$

Suponhamos que $d_{\varepsilon}=\left\{a=t_{0}<t_{1}<\ldots<t_{n}<t_{n+1}=b\right\}$. Como $F$ é pseudo-aditiva em $\left\{t_{1}, t_{2}, \ldots, t_{n}\right\}$, existe $\delta>0$ tal que, se $1 \leq i \leq n$, $0 \leq y_{i}^{\prime} \leq \delta, \quad 0 \leq y_{i}^{\prime \prime} \leq \delta$, tem-se:

$$
\left\|F\left(\left[t_{i}-y_{i}^{\prime}, t_{i}+y_{i}^{\prime \prime}\right]\right)-F\left(\left[t_{i}-y_{i}^{\prime}, t_{i}\right]\right)-F\left(\left[t_{i}, t_{i}+y_{i}^{\prime \prime}\right]\right)\right\| \leq \frac{\varepsilon}{n},
$$

$1 \leq \mathrm{i} \leq \mathrm{n}$.

Seja agora $d_{1}$ uma divisäo com $\Delta d_{1} \leq \delta$ e $d_{2}=d_{1} u d_{\varepsilon}$. Vamos comparar

$$
\sum_{i=1}^{\left|d_{2}\right|} F\left(I_{i}\right) \quad \text { e } \sum_{i=1}^{\left|d_{1}\right|} F\left(I_{i}\right)
$$

usando o mesmo valor para $F$ em todo intervalo de $d_{1}$ que não contêm pontos de $d_{\varepsilon}$ no seu interior. Um câlculo simplesmostra que

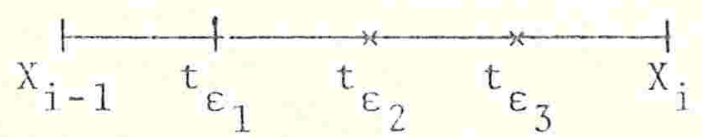

$$
\begin{aligned}
& \left\|\sum_{i=1}^{\left|d_{2}\right|} F\left(I_{i}\right)-\sum_{i=1}^{\left|d_{1}\right|} F\left(I_{i}\right)\right\| \leq \sum_{i=1}^{n} \| F\left(\left[t_{i}-y_{i}^{\prime}, t_{i}+y_{i}^{\prime \prime}\right]\right)- \\
& -F\left(\left[t_{i}-y_{i}^{\prime}, t_{i}\right]\right)-F\left(\left[t_{i}, t_{i}+y_{i}^{\prime \prime}\right]\right) \| \leq \varepsilon \text {. }
\end{aligned}
$$


Como $\mathrm{d}_{2} \geq \mathrm{d}_{\varepsilon}$ e $\Delta \mathrm{d}_{1} \leq \delta$, virá:

$$
\begin{aligned}
\| \sum_{i=1}^{\left|d_{1}\right|} F\left(\varepsilon_{i}\right) & -\sigma \int_{a}^{b} F\|\leq\| \sum_{i=1}^{\left|d_{1}\right|} F\left(\varepsilon_{i}\right)-\sum_{i=1}^{\left|d_{2}\right|} F\left(\varepsilon_{i}\right) \|+ \\
& +\left\|\sum_{i=1}^{\left|d_{2}\right|} F\left(\varepsilon_{i}\right)-\sigma \int_{a}^{b} F\right\| \leq \varepsilon+\varepsilon=2 \varepsilon .
\end{aligned}
$$

Logo, existe $N \int_{a}^{b} F$ e $N \int_{a}^{b} F=\sigma \int_{a}^{b} F$.

$\underline{\text { COROLÄRIO }} \underline{1 . \mathrm{b} .11}$ - Se $\mathrm{X}$ è um espaço de Banach e $\mathrm{f}:[\mathrm{a}, \mathrm{b}] \rightarrow \mathrm{X}$ então são equivalentes:

1) Existe $o \int_{a}^{b} f(x) d x$.

2) Existe $N \int_{a}^{b} f(x) d x$.

Temos ainda:

$$
\int_{a}^{b} f(x) d x=N \int_{a}^{b} f(x) d x=\sigma \int_{a}^{b} f(x) d x .
$$

DEMONSTRAÇĀO - E decorrência imediata do teorema $1 . b .10$ e da observação $1 . b \cdot 9$.

$\underline{\text { COROLARIO }} \underline{1 . b .12}$ - Se $x$ é um espaço de Banach e $f:[a, b] \rightarrow x$ são equivalentes:

1) $\lim _{\Delta d \rightarrow 0} w_{i}(f)\left[t_{i}-t_{i-1}\right]=0$.

2) $\lim _{\mathrm{d} \in \mathrm{D}} \omega_{i}(\mathrm{f})\left[\mathrm{t}_{i}-\mathrm{t}_{i-1}\right]=0$.

DEMONSTRACĀO - Imediata a partir do teorema $1 . b .10$ e da observação 1. b. 9 .

CQD

Como decorrência do corolärio $1 . b .11$ e do corılä- 
rio $1 . b .12$, os conceitos de integrabilidade segundo Riemann, $N \int_{a}^{b} f$ e $\sigma \int_{a}^{b} f$, são, na realidade, o conceito de $\int_{a}^{b} f(x) d x, \quad o$ mesmo ocorrendo com os conceitos de integrabilidade segundo Darboux, definidos em 1.b.4: ambos coincidem com o conceito definido em 1.a.8.

o teorema que se segue é uma caracterizaçãodas funções integráveis segundo Darboux e que sera $\vec{u} t i l$ na solução dos problemas propostos na introdução.

$\underline{\text { TEOREMA }} \underline{1 . \mathrm{b} .13}$ - Se $\mathrm{X}$ é um espaço de Banach e $\mathrm{f}:[\mathrm{a}, \mathrm{b}] \rightarrow \mathrm{X}$ são equivalentes 1) e 2) abaixo:

1) $f \in D([a, b], x)$.

2) a) $f \in B([a, b], X)$.

b) $u(D(f))=0$.

$\underline{\text { DEMONSTRACĀO }}-2) \Longrightarrow 1)-\operatorname{seja} M=\sup _{t, s \in[a, b]}\|f(t)-f(s)\| \cdot$ Então MEIR pois, por hipótese, $f \vec{e} 1$ imitada. Se $M=0$ então $f(t)=$ $=f(a)$, para todo $t \in[a, b]$ e não hä nada a demonstrar.Se $M \neq 0$, dado $\varepsilon>0$ tomemos

$$
\frac{1}{n_{0}}<\frac{\varepsilon}{2(b-a)} \text { e } F_{n_{0}}=\left\{t / \omega(\{t\}, f) \geq \frac{1}{n_{0}}\right\}
$$

Fi claro que $F_{n_{0}} \vec{c}$ un conjunto compacto, e portanto, mensurävel. Como $\mathrm{F}_{\mathrm{n}_{0}} \subset \mathrm{D}(\mathrm{f})$, concluímos que $\mathrm{u}\left(\mathrm{F}_{\mathrm{n}_{0}}\right)=0$. Da compacidade de $\mathrm{F}_{\mathrm{n}_{0}}$ e de $\mathrm{u}\left(\mathrm{F} \mathrm{n}_{0}\right)=$ $=0$ segue que existe uma divisão de $[\mathrm{a}, \mathrm{b}]$, que denominaremos $\mathrm{d}_{\varepsilon}$, tal que a soma das medidas dos intervilos cujos extremos sio pontos consecut ivos de $d_{\varepsilon}$ e interceptam $\mathrm{F}_{\mathrm{n}_{0}} \overrightarrow{\mathrm{e}}$ menor que $\frac{\varepsilon}{2 \mathrm{M}} ;$ nos restantes intervalos, 
cujos extremos são pontos de $d_{\varepsilon}$ temos que $\omega_{i}(f)<\frac{1}{n_{0}}$. Tomemos d, divisão de $[a, b]$ mais fina que $d_{E}$, e teremos:

$$
\sum_{i=1}^{|d|} \omega_{i}(f)\left[t_{i}-t_{i-1}\right]=\sum_{i \in A} \omega_{i}(f)\left[t_{i}-t_{i-1}\right]+\sum_{i \in B} \omega_{i}(f)\left[t_{i}-t_{i-1}\right],
$$

onde A é o conjunto dos indices dos pontos de d, extremos superiores de intervalos que interceptam $F_{n_{0}}$, e $B$ é o conjunto dos indices restantes não nulos. Então:

$$
\begin{gathered}
\sum_{i \in A} \omega_{i}(f)\left[t_{i}-t_{i-1}\right] \leq M \sum_{i \in A}\left[t_{i}-t_{i-1}\right] \leq M \cdot \frac{\varepsilon}{2 M}=\frac{\varepsilon}{2} . \\
\sum_{i \in B} \omega_{i}(f)\left[t_{i}-t_{i-1}\right] \leq \frac{1}{n_{0}} \sum_{i \in B}\left[t_{i}-t_{i-1}\right] \leq \frac{1}{n_{0}}(b-a) \leq \frac{\varepsilon}{2(b-a)}(b-a)=\frac{\varepsilon}{2} .
\end{gathered}
$$

Logo, se $d \geq d_{\varepsilon}$ temos

$$
\sum_{i=1}^{|d|} \omega_{i}(f)\left[t_{i}-t_{i-1}\right] \leq \varepsilon .
$$

Aplicando o corolário 1.b.12, virá que $f \in D([a, b], x)$.

1) $\Longrightarrow 2$ )

1) $\Longrightarrow 2 a)-E$ consequếncia da proposição $1 . b .6$, item 2 .

1) $\Longrightarrow 2 \mathrm{~b}$ ) - Como

$$
D(f)=\bigcup_{n=1}^{\infty} F_{n}, F_{n}=\left\{t / \omega(\{t\}, f) \geq \frac{1}{n}\right\},
$$

então $D(f)$ é mensurävel. Se $u[D(f)]>0$ existe um indice $\mathrm{n}_{0}$ tal que $u\left(F_{n_{0}}\right)>0$; portanto, qualquer que seja a divisão d $\in \mathcal{D}$, temos

$$
\sum_{i=1}^{|d|} \omega_{i}(f)\left[t_{i}-t_{i-1}\right] \geq \sum_{i \in A} \omega_{i}(f)\left(t_{i}-t_{i-1}\right),
$$


onde $A \vec{e}$ o conjunto de indices anteriormente definido. Mas

$$
\sum_{i \in A} \omega_{i}(f)\left[t_{i}-t_{i-1}\right] \geq \frac{1}{n_{0}} u\left(F_{n_{0}}\right)>0 .
$$

Logo, para todo d $E D$, temos

$$
\sum_{i=1}^{|d|} w_{i}(f)\left[t_{i}-t_{i-1}\right] \geqslant \frac{u\left(F_{n_{0}}\right)}{n_{0}}
$$

e $f \notin D([a, b], x):$ absurdo.

COROLĀRIO 1.b.14 - (Veja proposição 1.a.10).

1) $C([a, b], x) \subseteq D([a, b], X)$.

2) Se $f \in D([a, b], x)$ e $g \in C^{b}(x, z)$ então $g \circ f \in D([a, b], z)$.

3) Se $f \in D([a, b], x)$ então $\|f\| \in D([a, b], \mathbb{R})$.

4) Sejam $X, Y, Z$ espaços de Banach, B: XXY $\rightarrow Z$ uma ap 1 icação bilinear contínua, $f \in D([a, b], X)$ e $g \in D([a, b], Y)$. Escrevemos $t \stackrel{f \cdot g}{\longrightarrow} f(t) \cdot g(t)=B(f(t), g(t)) \cdot$ Então, $f \cdot g \in D([a, b], z)$.

5) Se A è uma älgebra de Banach então $D([a, b], A)$ è uma $\vec{a} 1$ gebra de Banach.

6) Se $f_{n} \in D([a, b], x)$ e $f_{n}$ converge uniformemente para $f$, então

$$
f \in D([a, b], x) \text { e } \int_{a}^{b} f_{n}(t) d t \stackrel{n \rightarrow \infty}{\longrightarrow} \int_{a}^{b} f(t) d t .
$$

7) Se fen $([a, b], X)$ e $F:[a, b] \longrightarrow X$ e dada por

$$
F(t)=\int_{a}^{t} f(x) d x
$$


então $F \vec{e}$ derivăvel quase sempre.

DEMONSTRAC,ĀO - 1) Se $f \in C([a, b], X)$ temos que $f \in B([a, b], X)$ e $u[D(f)]=u(\phi)=0$. Logo, $f \in D([a, b], X)$.

2) Basta observar que $g \circ f \in B([a, b], z)$ e, como $D(g \circ f) \subset D(f)$, vem que $u[D(g \circ f)]=0 . \operatorname{Logo}, f \in D([a, b], z)$.

3) Caso particular de 2) em que $g(x)=\|x\|$.

4) $E$ imediato, pois $f \cdot g \in B([a, b], Z)$ e $D(f \cdot g) \subset D(f) \cup D(g)$; $\operatorname{logo}, u[D(f \cdot g)]=0$ e então $f \cdot g \in D([a, b], z)$.

5) E consequência imediata de 4), lembrando que a composição è bilinear contínua.

6) Limite uniforme de funções 1imitadas ê uma função 1imitada; $\log o, f \in B([a, b], x)$. Por outro lado, $D(f) \subset \bigcup_{n=1}^{\infty} D\left(f_{n}\right)$ o que implica $u[D(f)]=0$ e $\operatorname{f\in D}([a, b], X)$.

Jä vimos que $f \in \Gamma(\lceil a, b], X)$ e è imediato que $f_{n}$ - $\operatorname{f\in D}([\mathrm{a}, \mathrm{b}], \mathrm{x})$. Logo, $\left\|\mathrm{f}_{\mathrm{n}}-\mathrm{f}\right\| \in \mathrm{E}([\mathrm{a}, \mathrm{b}], \mathbb{R})$ (corolärio 1.b.14, item 3) e, pela proposição $1 . a .9$ item $1,\left\|f_{n}-f\right\| \in R([a, b], \mathbb{R})$.

Como

$$
\left\|\int_{a}^{b} f_{n}(t) d t-\int_{a}^{b} f(t) d t\right\| \leq \int_{a}^{b}\left\|f_{n}-f\right\| d t
$$

e, dado $\varepsilon>0$, existe $n_{0}$ tal que, se $n \geq n_{0}$ temos $\left\|f_{n}(t)-f(t)\right\| \leq$ $\leq \frac{\varepsilon}{b-a}$, para todo $t \in[a, b]$ então:

$$
n \geq n_{0} \Rightarrow\left\|\int_{a}^{b} f_{n}(t) d t-\int_{a}^{b} f(t) d t\right\| \leq \varepsilon, \quad e \lim _{n \rightarrow \infty} \int_{a}^{b} f_{n}(t) d t=\int_{a}^{b} f(t) d t .
$$


I.c - NOÇŌES SOBRE FUNÇŌES REGRADAS

DEFINIÇÄO 1.c.1 - Seja X um espaço de Banach. Denotaremos por $G([a, b], X)$ o conjunto das funções $f:[a, b] \rightarrow X$ regradas, isto $\vec{e}$, tais que existem os 1 imites laterais de $f$ em todo ponto $t \in[a, b]$.

Funções regradas säo descritas pela seguinte proposição.

$\underline{\text { PROPOSIÇĀO }} \underline{1 . c \cdot 2}$ - Se X è um espaço de Banach e $f:[a, b] \rightarrow X$, então são equivalentes:

1) $f \in G([a, b], x)$.

2) $f \vec{e}$ limite uniforme de funções em escada.

3) Para todo $\varepsilon>0$, existe deD tal que

$$
\left.\omega(] t_{i-1}, t_{i} \Gamma, f\right) \leq \varepsilon, \quad 1 \leq i \leq n .
$$

DEMONSTRAÇĀO - Veja [H-3], pägina 20 , teorema 3.1 .

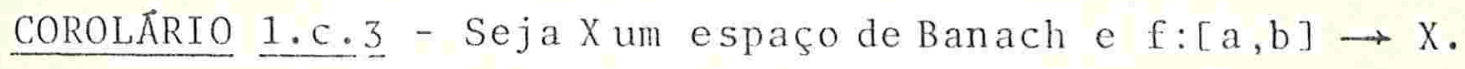
1) Se $f \in G([a, b], x)$ entāo:
a) $\mathrm{f} \in \mathrm{B}([\mathrm{a}, \mathrm{b}], \mathrm{x})$;
b) $D(f) \vec{e} e-$ numeräve1.

2) $\mathcal{C}([\mathrm{a}, \mathrm{b}], \mathrm{X}) \subset \mathrm{G}([\mathrm{a}, \mathrm{b}], \mathrm{X})$.

3) $G([a, b], X) \subset D([a, b], X)$.

4) Se $f \in G([a, b], X)$, existe $g \in C([a, b], X)$ deriväve1 a menos de um conjunto enumerävel $N=D(f)$, e tal que: 
a) $g^{\prime}(t)=f(t)$, para todo $t \in S=[a, b]-N$ e $g_{+}^{\prime}\left(t^{+}\right)=f\left(t^{+}\right), g_{-}^{\prime}\left(t^{-}\right)=f\left(t^{-}\right)$.

b) Para todo $t_{0} \in[a, b]$ temos

$$
\left\|g(b)-\oint(a)-f\left(t_{0}\right)(b-a)\right\| \leq(b-a) \sup _{s \in S}\left\|f(s)-f\left(t_{0}\right)\right\| \text {. }
$$

\section{DEMONSTRACĀO -}

1) a) Pela proposição 1.c.2, item 2, as funções regradas são limites uniformes de funções 1 imitadas; 10 go, são 1 imitadas.

b) Temos que

$$
D(f)=\bigcup_{n=1}^{\infty} F_{n}, \operatorname{com} F_{n}=\left\{t / \omega(\{t\}, f) \geq \frac{1}{n}\right\} \text {. }
$$

Pela proposição 1.c.2, item 3, cada um dos $F_{n}$ éfinito, e portanto, $D(f) \vec{e}$ enumeräve1.

2) Imediata da definição de função regrada.

3) E decorrência do item 1 deste corolärio e do teorema $1 . b .13$, item $2 b$, pois, se um conjunto $\vec{e}$ enumeräve 1 , tem medida nula.

4) a) Veja [D], 8.7.2. b) Veja [H-1], pägina 63, c8.

1.d-NOÇŌES SOBRE FUNÇŌES DE VARIAÇĀO FORTE LIMITADA, VARIAÇĀO FRACA LIMITADA E SEMIVARIAÇĀO LIMITADA

$\underline{\text { DEFINIÇĀO }} \underline{1 . \mathrm{d} .1}$ - Dado $X$ um espaço de Banach, $f:[\mathrm{a}, \mathrm{b}] \rightarrow \mathrm{X}$, e $d \in D$, denotamos por $V_{d}[f]=\sum_{i=1}^{|d|}\left\|f\left(t_{i}\right)-f\left(t_{i-1}\right)\right\|$. 
Diremos que $\mathrm{f} \bar{e}$ de variação forte limitada, e escrevemos

$$
f \in B V([a, b], x) \text {, se } V[f]=\sup _{d \in D} V_{d}[f]<\infty .
$$

$\underline{\text { DEFINIÇĀO }} \underline{1 . d .2}$ - Dado $X$ um espaço de Banach, $f:[a, b] \rightarrow X$ e $\mathrm{d} \in \mathcal{D}$, denotaremos por

$$
W_{d}[f]=\sup _{\left|\lambda_{i}\right| \leq 1}\left\|\sum_{i=1}^{|d|}\left[f\left(t_{i}\right)-f\left(t_{i-1}\right)\right] \lambda_{i}\right\| .
$$

Diremos que $\mathrm{f}$ é de variação fraca limitada, e escrevemos

$$
f \in B W([a, b], X) \text {, se } W[f]=\sup _{d \in D} W_{d}[f]<\infty \text {. }
$$

$\underline{\text { DEFINIÇÃO }} \underline{1 . d .3}$ - Dados $X$ um espaço de Banach, $f:[a, b] \rightarrow X$ e $\delta=\left\{a \leq s_{1}<t_{1} \leq s_{2}<t_{2} \leq \ldots \leq s_{n}<t_{n} \leq b\right\}$, denotaremos por

$$
w_{\delta}[f]=\left\|\sum_{i=1}^{n}\left[f\left(t_{i}\right)-f\left(s_{i}\right)\right]\right\|
$$

Diremos que $\underline{f \in B \omega([a, b], X)}$ se $\omega[f]=\sup _{\delta \in \Delta} \omega_{\delta}[f]<\infty$.

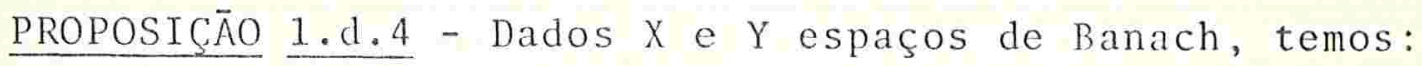

1) a) $B V([a, b], X) \subset C([a, b], X) \subset B([a, b], X)$.

b) $B V([a, b], X) \subset B W([a, b], X) \subset B([a, b], X)$.

c) $B V([a, b], x) \subset B W([a, b], X) \subset B([a, b], X)$.

2) a) Se $f \in B V([a, b], X)$ e $u \in L(X, Y)$ então

$$
u \circ f \in B V([a, b], Y) \text { e } V[u \circ f] \leq\|u\| V[f] .
$$

b) $\operatorname{Se} f \in B W([a, b], X)$ e $u \in L(X, Y)$ então

$$
u \circ f \in B(w([a, b], X) \text { e } w[u \circ f] \leq\|u\| \omega[f] \text {. }
$$


c) Se $f \in B W([a, b], X)$ e $u \in L(X, Y)$ então $u \circ f \in B W([a, b], x)$ e $W[u \circ f] \leq\|u\| W[f]$.

3) Se J: X $\longrightarrow$ Y é um isomorfismo linear, são equivalentes: a) e $\left.a^{\prime}\right)$, b) e $b^{\prime}$ ), bem como c) e $c^{\prime}$ ).

a) $\left.f \in B V([a, b], X) ; \quad a^{\prime}\right) \quad J \circ f \in B V([a, b], Y)$.

b) $\left.f \in B W([a, b], X) ; \quad b^{\prime}\right) J \circ f \in B W([a, b], Y)$.

c) $\left.f \in B W([a, b], x) ; \quad c^{\prime}\right) \quad J \circ f \in B W([a, b], Y)$.

4) a) $\operatorname{BV}([a, b], X)$ só depende da topologia de $X$.

b) $B W([a, b], X)$ só depende da topologia de $X$.

c) $\operatorname{BIV}([a, b], x)$ só depende da topologia de $x$.

5) Se $\left\{x_{i}\right\}_{i=1}^{n}$ são espaços de Banach, $x=\prod_{i=1}^{n} x_{i} e$ $\mathrm{P}_{i}: x \rightarrow x_{i}, 1 \leq \mathrm{i} \leq \mathrm{n}$, è tal que se $\mathrm{x}=\left(\mathrm{x}_{1}, \mathrm{x}_{2}, \mathrm{x}_{3}, \ldots, \mathrm{x}_{\mathrm{n}}\right)$, $P_{i}(x)=x_{i}, \quad 1 \leq i \leq n$, são equivalentes a) e $a^{\prime}$, b) e $b^{\prime}$ ) bem como c) e $c^{\prime}$ ).

a) $\left.f \in B V([a, b], x) ; a^{\prime}\right) f_{i}=P_{i} \circ \in \in B V\left([a, b], x_{i}\right) ; 1 \leq i \leq n$.

b) $f \in B\left(\omega([a, b], x) ; b^{\prime}\right) f_{i}=P_{i} \circ \in \in B \omega\left([a, b], x_{i}\right) ; 1 \leq i \leq n$.

c) $\left.f \in B W([a, b], x) ; c^{\prime}\right) f_{i}=p_{i} \circ f \in B W\left([a, b], x_{i}\right) ; 1 \leq i \leq n$.

6) $\operatorname{BW}([a, b], X) \subset R([a, b], x)$.

DEMONSTRAÇĀO - 1) a) Para a prova de BV $([a, b], X) \subset G([a, b], X)$, veja $[\mathrm{H}-1]$, pägina $63, \mathrm{C}-5$.

$G([a, b], X) \subset B([a, b], X)$ está demonstrado no corolário 1.c.3, 1) a) .

b) $\left\|\sum_{i=1}^{n}\left[f\left(t_{i}\right)-f\left(s_{i}\right)\right]\right\| \leq \sum_{i=1}^{n}\left\|f\left(t_{i}\right)-f\left(s_{i}\right)\right\| \leq v[f] ; \log 0$ 
$W[f] \leq V[f]$. Dai segue imediatamente que, se $f \in B V([a, b], X)$ então $\operatorname{f\in B} W([a, b], X)$.

Se $f \in B W([a, b], x)$, vem que $\|f(t)-f(a)\| \leq w[f]$, para todo $t \in[a, b]$ Então, $f \in B([a, b], x)$.

c) Anäloga a de b).

2) a) Se $f \in B V([a, b], X)$ e $u \in L(X, Y)$, temos:

$$
\sum_{i=1}^{|d|}\left\|(u \circ f)\left(t_{i}\right)-(u \circ f)\left(t_{i-1}\right)\right\| \leq\|u\|\left(\sum_{i=1}^{|d|}\left\|f\left(t_{i}\right)-f\left(t_{i-1}\right)\right\|\right) \leq\|u\| v[f] .
$$

$\operatorname{Logo}, \quad u \circ f \in B V([a, b], Y)$ e $V[u \circ f] \leq\|u\| V[f]$.

b) e c) Anālogas à de 2) a) desta proposição.

3) Consequência imediata do item 2 .

4) Consequência imediata do item 3 .

5) a) $\Longrightarrow a^{\prime}$ ) Consequência imediata do item 2.a) desta proposição.

$\left.\mathrm{a}^{\prime}\right) \Longrightarrow$ a) Tomemos em

$$
x=\prod_{i=1}^{n} x_{i},\|x\|=\left\|\left(x_{1}, x_{2}, \ldots, x_{n}\right)\right\|=\sum_{i=1}^{n}\left\|x_{i}\right\|_{i} .
$$

Então vem imediatamente que $V[f]=\sum_{i=1}^{n} V\left[f_{i}\right] ; \operatorname{logo}$, se

$$
f_{i} \in B V\left([a, b], x_{i}\right), \operatorname{temos} f \in B V([a, b], x) \text {. }
$$

b) $\left.\Longrightarrow b^{\prime}\right)$ Consequência imediata do item $\left.2 . b\right)$ desta proposição.

$\left.b^{\prime}\right) \Longrightarrow$ b) Tomemos, em $x=\prod_{i=1} x_{i}$, a norma $\|x\|=\sup _{1 \leq i \leq n}\left\|x_{i}\right\|_{i}$. Então vem imediatamente que $w[f]=\sup _{1 \leq i \leq n} \omega\left[f_{i}\right] ; \operatorname{logo}$, se 
$f_{i} \in B W\left([a, b], x_{i}\right)$, vem que $f \in B W([a, b], x)$.

c) $\left.\Longleftrightarrow c^{\prime}\right)$ Análoga à demonstração de 5) b) $\Longleftrightarrow b^{\prime}$ ) desta proposição.

6) Veja $[\mathrm{H}-1]$, pāgina 55 , teorema $\mathrm{A}-3$.

$\underline{\text { PROPOSIÇĀO }} \underline{1 . d .5}$ - 1) Se $\operatorname{fEBV}([\mathrm{a}, \mathrm{b}], \mathbb{C})$ então temos:

a) $W_{d}[f]=V_{d}[f]$ e $W[f]=V[f]$.

b) $\operatorname{BV}([\mathrm{a}, \mathrm{b}], \mathbb{C})=\operatorname{BW}([\mathrm{a}, \mathrm{b}], \mathbb{C})$.

2) a) Se $f:[a, b] \rightarrow \mathbb{R}$ então $\omega[f] \leq V[f] \leq 2 \omega[f]$.

b) Se $f:[a, b] \rightarrow \mathbb{C}$ então $w[f] \leq V[f] \leq 4 \omega[f]$.

c) $B(U(\lceil a, b], \mathbb{K})=B W(\lceil a, b], \mathbb{K})=B V([a, b\rceil, \mathbb{K})$ se $\mathbb{K}=\mathbb{R}$ ou $\mathbb{C}$.

3) Se X e um espaço de Banach de dimensão finita, temos que $\operatorname{BV}([a, b], X)=B W([a, b], X)=B W([a, b], X)$.

DEMONSTRAÇĀO - 1) a)

$$
\begin{aligned}
& V_{d}[f]=\sum_{i=1}^{|d|}\left|f\left(t_{i}\right)-f\left(t_{i-1}\right)\right|=\sum_{i=1}^{|d|}\left[f\left(t_{i}\right)-f\left(t_{i-1}\right)\right] \lambda_{i} \leq \\
& \left|\lambda_{i}\right| \leq 1 \text {. } \\
& \text { ( } \lambda_{i} \text { convenientemente escolhidos) } \\
& \leq \sup _{\left|\lambda \operatorname{li}_{i}\right| \leq 1}\left|\sum_{i=1}^{|d|}\left[f\left(t_{i}\right)-f\left(t_{i-1}\right)\right] \lambda_{i}\right|=w_{d}[f],
\end{aligned}
$$

donde vem que $V_{d}[f] \leq W_{d}[f]$.

por sua vez,

$$
w_{d}[f]=\sup _{\left|\lambda_{i}\right| \leq 1}\left|\sum_{i=1}^{|d|}\left[f\left(t_{i}\right)-f\left(t_{i-1}\right)\right] \lambda_{i}\right| \leq \sum_{i=1}^{|d|}\left|f\left(t_{i}\right)-f\left(t_{i-1}\right)\right|=v_{d}[f] ;
$$

$\log 0, W_{d}[f]=V_{d}[f]$. 
Tomando o supremo quando $\mathrm{d} \in D$, virä $\mathrm{W}[\mathrm{f}]=\mathrm{V}[\mathrm{f}]$.

b) Consequência imediata do item a.

2) a) Para $W[f] \leq V[f]$, veja demonstração da proposição 1.d.4, item b).

Dada uma divisão $d=\left\{a=t_{0}<t_{1}<\ldots<t_{n}=b\right\}$, chamemos $P$ o conjunto dos indices $i$ tais que $f\left(t_{i}\right)-f\left(t_{i-1}\right) \geq 0$ e No conjunto dos indices restantes. Temos:

$$
\begin{aligned}
V_{d}[f] & =\sum_{i=1}^{|d|}\left|f\left(t_{i}\right)-f\left(t_{i-1}\right)\right|= \\
& \left.=\sum_{i \in P} \mid f\left(t_{i}\right)-f\left(t_{i-1}\right)\right]-\sum_{i \in N}\left[f\left(t_{i}\right)-f\left(t_{i-1}\right)\right] \leq 2 w[f] .
\end{aligned}
$$

$\log o, V[f]=2 w[f]$.

b) Decorrência imediata de 2)a) desta proposição.

c) Decorrência de 2) a), b) e 1)b) desta proposição.

3) Se $x$ ê um espaço de Banach de dimensão fínita, então existe um isomorfismo $J: X \longrightarrow \mathbb{K}^{\mathrm{n}}(\mathbb{K}=\mathbb{R}$ ou $\mathbb{C})$. Então $f \in B N([a, b], x) \stackrel{\left.1 \cdot(\cdot 4 \cdot 3) c) \Leftrightarrow c^{i}\right)}{\Longleftrightarrow} \operatorname{JofEBW}\left([a, b], \pi K^{n}\right)$ $\stackrel{\left.1 . d .4 .5) c) \Leftrightarrow c^{\prime}\right)}{\Longleftrightarrow} P_{i} \circ \operatorname{J} \circ \operatorname{fEBN}([a, b], \mathbb{K}) \stackrel{1 . d .5 .2) c)}{=} \operatorname{BV}([a, b], \mathbb{K})$ $\stackrel{\left.1 \cdot d \cdot 4 \cdot 5) a) \Longleftrightarrow a^{1}\right)}{\Longleftrightarrow} J \circ f \in B V\left([a, b], \mathbb{K}^{n}\right) \stackrel{\left.1, d \cdot 4.3) a) \Longleftrightarrow a^{\prime}\right)}{\Longleftrightarrow} f \in B V([a, b], x)$. A demonstraçã̃o para $B(W$ è análoga à anterior.

o teorema seguinte mostra três maneiras equivalentes de se defirir funçōes de variação fraca limitada, e que 
nos serão üteis em futuras aplicações.

$\underline{\text { TEOREMA }} \underline{1 . \mathrm{d} .6}$ - Se $\mathrm{X}$ é um espaço de Banach e $\mathrm{f}:[\mathrm{a}, \mathrm{b}] \longrightarrow \mathrm{X}$, são equivalentes:

1) $f \in B W([a, b], x)$.

2) Para todo $x^{\prime} \in X^{\prime}, x^{\prime} \circ f \in B V([a, b], \mathbb{K}) \quad(\mathbb{K}=\mathbb{R}$ ou $\mathbb{C})$.

3) $f \in B w([a, b], x)$.

Alêm disso, $W_{d}[f]=\sup _{\left\|x^{\prime}\right\| \leq 1} V_{d}\left[x^{\prime} \circ f\right] ; \operatorname{logo}, W[f]=\sup _{\left\|x^{\prime}\right\| \leq 1} V\left[x^{\prime} \circ f\right]$. DEMONSTRAÇĀO -1$) \Longrightarrow 3$ ) Se $\delta=\left\{a \leq s_{1}<t_{1} \leq \ldots \leq s_{n}<t_{n} \leq b\right\}$, e dé a partição induzida em $[\mathrm{a}, \mathrm{b}]$ por $\delta \mathrm{e}$

$$
\lambda_{j}=\left\{\begin{array}{l}
1 \text { se } t_{j-1}=s_{i} \quad e / t_{j}=t_{i} \\
0 \quad \text { em caso contrário }
\end{array},\right.
$$

temos:

$$
w_{d}[f]=\left\|\sum_{i=1}^{n}\left[f\left(t_{i}\right)-f\left(s_{i}\right)\right]\right\|=\left\|\sum_{i=1}^{|d|}\left[f\left(t_{j}\right)-f\left(t_{j-1}\right)\right] \lambda_{i}\right\| \leq w_{d}[f] \leq w[f] .
$$

$\operatorname{Logo}$, se $f \in B W([a, b], X)$, virä que $f \in B W([a, b], X)$.

$3) \Longrightarrow 2)$ Se $f \in B W([a, b], X)$, pela proposição $1 . d \cdot 4,2)$ b),

$$
x^{\prime} \circ f \in B W([a, b], \mathbb{K})=B V([a, b], \mathbb{K}) \text {. }
$$

2) $\Longrightarrow 1)$ Denotemos por $\lambda=\left(\lambda_{1}, \lambda_{2}, \ldots, \lambda_{|d|}\right)$. Fixada $f$ nas condições de 2), seja $A_{\lambda, d}: X^{\prime} \rightarrow \mathbb{K}$ a aplicação dada por

$$
A_{\lambda, d}\left(x^{\prime}\right)=x^{\prime}\left(\sum_{i=1}^{|d|}\left[f\left(t_{i}\right)-f\left(t_{i-1}\right) \lambda_{i}\right]\right)
$$


Tal aplicação $\vec{e}$ linear e continua. Alëm disso:

$$
\begin{aligned}
&\left|x^{\prime}\left(\sum_{i=1}^{|d|}\left[f\left(t_{i}\right)-f\left(t_{i-1}\right)\right] \lambda_{i}\right)\right|=\left|\sum_{i=1}^{|d|}\left[\left(x^{\prime} \circ f\right)\left(t_{i}\right)-\left(x^{\prime} \circ f\right)\left(t_{i-1}\right)\right] \lambda_{i}\right| \leq \\
& \leq \sum_{i=1}^{|d|}\left|\left(x^{\prime} \circ f\right)\left(t_{i}\right)-\left(x^{\prime} \circ f\right)\left(t_{i-1}\right)\right| \leq V\left[x^{\prime} \circ f\right],\left|\lambda_{i}\right| \leq 1
\end{aligned}
$$

e dED.

Então, pelo princípio de limitação uniforme,

$$
\sup _{d \in D} \sup _{i}\left\{\left\|\sum_{i=1}^{|d|}\left[f\left(t_{i}\right)-f\left(t_{i-1}\right)\right] \lambda_{i}\right\|\right\}<\infty
$$

ou $W[f]<\infty . \log o, f \in B N([a, b], X)$. Finalmente,

$$
\begin{aligned}
& W_{d}[f]=\sup _{\left|\lambda_{i}\right| \leq 1}\left\|\sum_{i=1}^{|d|}\left[f\left(t_{i}\right)-f\left(t_{i-1}\right)\right] \lambda_{i}\right\|= \\
& \stackrel{\text { Hahn-Banach }}{=} \sup _{\left|\lambda_{i}\right| \leq 1} \sup _{\left\|x^{\prime}\right\| \leq 1}\left|\sum_{i=1}^{|d|}\left[\left(x^{\prime} \circ f\right)\left(t_{i}\right)-\left(x^{\prime} \circ f\right)\left(t_{i-1}\right)\right] \lambda_{i}\right|= \\
& =\sup _{\left\|x^{\prime}\right\| \leq 1} \sup _{\left|\lambda_{i}\right| \leq 1}\left|\sum_{i=1}^{|d|}\left[\left(x^{\prime} \circ f\right)\left(t_{i}\right)-\left(x^{\prime} \circ f\right)\left(t_{i-1}\right)\right] \lambda_{i}\right|= \\
& =\sup _{\left\|x^{\prime}\right\| \leq 1} W_{d}\left[x^{\prime} \circ f\right] \stackrel{1 . d \cdot 5 \cdot 1) a)}{=} \sup _{\left\|x^{\prime}\right\| \leq 1} V_{d}\left[x^{\prime} \circ f\right]
\end{aligned}
$$

Então,

$$
w_{d}[f]=\sup _{\left\|x^{\prime}\right\| \leq 1} V_{d^{\prime}}\left[x^{\prime} \circ f\right] \quad \text { e } w[f]=\sup _{\left\|x^{\prime}\right\| \leq 1} V\left[x^{\prime} \circ f\right] .
$$

$\underline{\text { DEFINICŌES }} \underline{1 . d .7}$ - a) Dada $\mathrm{f}:[\mathrm{a}, \mathrm{b}] \longrightarrow \mathrm{L}(\mathrm{X}, \mathrm{Y})$, para todo deD escrevemos 


$$
\operatorname{SV}_{d}[f]=\sup \left\{\left\|\sum_{i=1}^{|d|}\left[f\left(t_{i}\right)-f\left(t_{i-1}\right)\right] \cdot x_{i}\right\| / x_{i} \in X,\left\|x_{i}\right\| \leq 1\right\},
$$

e definimos semivariação de $f$ em $[a, b]$ por $S V[f]=\sup _{d \in D} S V_{d}[f]$. 0 conjunto das funções $f:[a, b] \rightarrow L(X, Y)$ tais que $S V[f]<\infty$ será denotado por $\operatorname{SV}([a, b], L(X, Y))$.

b) Dados $X$ e $Y$ espaços de Banach, denotaremos por:$$
\operatorname{CSV}([a, b], L(X, Y))=\mathcal{C}([a, b], L(X, Y)) \cap S V([a, b], L(X, Y)) .
$$

c) Observemos ainda que, se $x=\mathbb{K}(\mathbb{R}$ ou $\mathbb{C})$, temos:

$$
\operatorname{SV}([a, b], L(X, Y))=B W([a, b], Y) .
$$

(Veja teorema 1.d.6).

1.e-PROBLEMAS E EXEMPLO

\section{$\underline{1 . e .1}-\underline{\text { PROBLEMAS }}$}

Propomo-nos agora resolver os problemas $[P-n], 1 \leq n \leq 8$, aos quais nos referimos na introdução. Daremos, no capítulo 1, a resolução do problema [P-1]. Resolveremos e discutiremos os restantes no capitulo 3 .

$\underline{1 . e .2}-\underline{\text { EXEMPLO }}$

Se ja

$$
C_{0}(\mathbb{N})=\left\{\left(x_{i}\right){ }_{i \in \mathbb{N}} / x_{i} \in \mathbb{K} \text { e } \lim _{i \rightarrow \infty} x_{i}=0,\|x\|=\sup _{i \in \mathbb{N}}\left|x_{i}\right|\right\} .
$$

Consideremos uma enumeração dos racionais de $[a, b]$ : 
$\left\{r_{i}\right\}_{i=1}^{\infty}$. Vamos denotar

$$
\begin{gathered}
\ell_{1}=(1,000 \ldots) \ldots \ell_{i}=(0,0, \ldots 100 \ldots) \ldots \\
\text { Definimos } f:[a, b] \longrightarrow c_{0}(\mathbb{N}) \text { por } \\
f(x)= \begin{cases}l_{i} \text { se } x=r_{i} \\
0 \text { se } x \notin \mathbb{Q}[a, b] .\end{cases}
\end{gathered}
$$

Observe-se que:

1) $f \in B W\left([a, b], c_{0}(\mathbb{N})\right)$, e portanto,

2) $\operatorname{f\in R}\left([a, b], C_{0}(\mathbb{N})\right)$.

3) $f \notin D\left([a, b], C_{0}(\mathbb{N})\right)$, e portanto,

4) $f \notin G\left([a, b], c_{0}(\mathbb{N})\right)$ e

5) $f \notin B V\left([a, b], c_{0}(\mathbb{N})\right)$.

(Logo, $f$ mostra que tem sentido os problemas [P-1], [P-2], [P-3] e [P-5]).

DEMONSTRAC $\bar{A} O-1)$ seja $\delta=\left\{a \leq s_{1}<t_{1} \leq s_{2}<t_{2} \leq \ldots \leq s_{n}<t_{n} \leq b\right\} . \quad E \quad i-$ mediato que

$$
\left\|\sum_{i=1}^{n}\left[f\left(t_{i}\right)-f\left(s_{i}\right)\right]\right\|_{\infty} \leq 1
$$

$\mathrm{e}$ ainda $\omega[f]=1$.

2) Demonstraremos que $\int_{a}^{b} f(x) d x=0$. De fato,

$$
\left\|\sum_{i=1}^{|d|} f\left(\varepsilon_{i}\right)\left(t_{i}-t_{i-1}\right)-0\right\| \leq \Delta d
$$


Portanto, se $\Delta d \leq \delta=\varepsilon$, virá que

$$
\left\|\sum_{i=1}^{|d|} f\left(\varepsilon_{i}\right)\left(t_{i}-t_{i-1}\right)-0\right\| \leq \varepsilon, \quad e \int_{a}^{b} f(t) d t=0
$$

3) $f \notin D\left([a, b], c_{0}(\mathbb{N})\right)$, pois $f$ é descontínua em todo ponto do intervalo $[a, b], j \vec{a}$ que $\omega\left(\left[x_{0}-\delta, x_{0}+\delta\right], f\right) \geq\left\|e_{i}\right\|=1$, para todo $\delta>0$. Logo $u[D(f)]=b-a$, e então, pelo teorema 1.b.13, item $2, f \notin D\left([a, b], C_{0}(\mathbb{N})\right)$.

4) e 5) são decorrências imediatas de 3) pois, se f pertencesse a um dos espaços $G\left([a, b], C_{0}(\mathbb{N})\right)$ ou $B V\left([a, b], C_{0}(\mathbb{N})\right)$, pertenceria a $\mathrm{D}\left([\mathrm{a}, \mathrm{b}], \mathrm{C}_{0}(\mathbb{N})\right)$, o que contraria 3$)$.

\section{1.f - TEOREMA DE DVORETZKY-ROGERS}

Neste item apresentamos um resumo dos resultados necessärios para a solução do problema um.

$\underline{\text { PROPOSIC } \bar{A} O}$ 1.f.1 - Seja X um espaço de Banach e $\left\{x_{n}\right\}_{n=1}^{\infty}$ uma sequência de vetores em $x$. Säo equivalentes:

1) A sẻrie $\sum_{n=1}^{\infty} x_{\pi(n)}$ converge, para toda permutação $\pi$ de inteiros positivos.

2) A série $\sum_{i=1}^{\infty} x_{n_{i}}$ converge, para toda escolha $n_{1}<n_{2}<n_{3}<$ $<\ldots$.

3) A série $\sum_{n=1}^{\infty} \theta_{n} x_{n}$ converge, para toda escolha de sinais (isto $\overrightarrow{\mathrm{e}}, \theta_{\mathrm{n}}= \pm 1$ ). 
4) A sërie $\sum_{n=1}^{\infty} \theta_{n} x_{n}$ converge, para toda escolha de $\left\{\theta_{n}\right\}_{n=1}^{\infty}$ $\operatorname{com}\left|\theta_{n}\right| \leq M, n \in N$.

5) Para todo $\varepsilon>0$, existe um $n$ tal que $\left\|\sum_{i \in \sigma} x_{i}\right\| \leq \varepsilon$, para todo conjunto finito o de inteiros que satisfaz

$$
\min \{i \in \sigma\}>n \text {. }
$$

DEMONSTRAC.ĀO - Veja [L-T], pägina 15 , proposição $1 . c .1$ e [M-T], pägina 20 .

DEFINIÇAOO $1 . f .2$ - Num espaço de Banach $x$, se uma sërie $\sum_{n=1}^{\infty} x_{n}$ satisfaz uma das condições da proposição 1.f.1, então ela ẻ dita incondicionalmente convergente.

DEFINICĀO 1.f.3 - Uma série $\sum_{n=1}^{\infty} x_{n}$ num espaço de Banach $x$ ë dita absolutamente convergente se $\sum_{n=1}^{\infty}\left\|x_{n}\right\|<\infty$.

$\underline{\text { PROPOSIÇĀO }}$ 1. .4 (Riemann) - Se X é um espaço de Banach de dimensão finita, então são equivalentes:

1) $\sum_{n=1}^{\infty} x_{n} \vec{e}$ incondicionalmente convergente.

2) $\sum_{n=1}^{\infty} x_{n}$ ë absolutamente convergente.

DEMONSTRAC̄̄A - Veja $[M-T]$, pägina 22 , teorema 2.

Se $x$ tem dimensão infinita, Dvoretzky e Rogers demonstraram:

TEOREMA $\underline{1 . f .5}$ - Seja X um espaço de Banach de dimensão infinita. Seja $\left\{\lambda_{n}\right\}_{n=1}^{\infty}$ uma sequência de reais positivos tal que $\sum_{n=1}^{\infty}\left|\lambda_{i}\right|^{2}<\infty$. Então existe uma série $\sum_{n=1}^{\infty} x_{n}$ incondicional- 
mente convergente em $x$ tal que $\left\|x_{n}\right\|=\lambda_{n}$, para todo $n$. $\underline{\text { DEMONSTRACĀO }}$ - Veja $[\mathrm{D}-\mathrm{R}]$.

COROLÅRIO 1.f.6 (Dvoretzky-Rogers) - Seja X um espaço de Banach de dimensão infinita. Existe uma sërie $\sum_{n=1}^{\infty} x_{n}$ que é incondicionalmente convergente e não $\vec{e}$ absolutamente convergente.

DEMONSTRACĀO - Basta tomar $\lambda_{n}=\frac{1}{n}, n=1,2, \ldots$ e ap1icar o teorema $1 . f .5$.

o corolário abaixo mostra uma caracterização dos espaços de dimensão finita (ou infinita), dada por meio de séries e que ê consequência imediata do teorema do Dvoretzky-Rogers .

COROLÅRIO I.f.7 - Seja X um espaço de Banach. São equivalentes:

1) A dimensão de $x$ ê finita.

2) Toda série incondicionalmente convergente em $x \vec{e} a b-$ solutamente convergente em $x$.

DEMONSTRAÇĀO -1$) \Longrightarrow$ 2) Vide proposição 1.f.4.

2) $\Longrightarrow$ 1) vejir corolário 1.f.6.

CQD

1.g - PROBLEMA UM

Recordemos, mais uma vez, o denominado problema um [P-1]. 
$\underline{\text { PROBLEMA }} 1$ (P-1) - Caracterizar os espaços de Banach $X$ tais que $B(V([a, b], X)=B V([a, b], X)$.

0 teorema que se segue esclarece completamente a questão, alêm de dar uma caracterização dos espaços de Banach, de dimensão finita, por intermédio das funções de variação forte e fraca limitadas.

TEOREMA 1.g.1 - Seja X um espaço de Banach. São equivalentes:

1) A dimensão de $x$ é finita.

2) $B V([a, b], x)=B(W([a, b], x)$.

DEMONSTRAC.ĀO -1$) \Longrightarrow 2$ ) - Estā demonstrado na proposição 1.d.5, item 3 .

2) $\Longrightarrow 1$ ) - Faremos a demonstração para o caso $[a, b]=[0,1]$. Suponhamos $X$ espaço de Banach de dimensão infinita.Pelo corolärio $1 . f .6$ do teorema Dvoretzky-Rogers, existe uma série $\sum_{n=1}^{\infty} x_{n}$ que converge incondicionalmente, mas não converge absolutamente. Como

$$
[0,1]=\left\{\bigcup _ { j = 1 } ^ { \infty } \left[1-\frac{1}{j}, 1-\frac{1}{j+1}[\} \cup\{1\},\right.\right.
$$

seja $f:[0,1] \longrightarrow X$ definida por

$$
f(x)=\left\{\begin{array}{l}
0 \text { se } x=1 \\
x_{1}+x_{2}+\ldots+x_{j} ; \text { se } x \in\left[1-\frac{1}{j}, 1-\frac{1}{j+1}[.\right.
\end{array}\right.
$$


Afirmamos que $f \in B(W([0,1], X)$. De fato: seja,

$$
\delta=\left\{0 \leq \mathrm{s}_{1}<\mathrm{t}_{1} \leq \mathrm{s}_{2}<\mathrm{t}_{2} \leq \ldots \leq \mathrm{s}_{\mathrm{k}}<\mathrm{t}_{\mathrm{k}} \leq 1\right\} .
$$

Temos:

$$
\left\|\sum_{i=1}^{k}\left[f\left(t_{i}\right)-f\left(s_{i}\right)\right]\right\| \leq\left\|x_{n_{1}}+x_{n_{2}}+\ldots+x_{n_{\ell}}\right\|+\left\|x_{1}+x_{2}+\ldots+x_{m}\right\|
$$

onde $\mathrm{n}_{1}<\mathrm{n}_{2}<\ldots<\mathrm{n}_{\ell}$ e mEN.

Por outro lado, como $\sum_{n=1}^{\infty} x_{n} \vec{e}$ incondicionalmente convergente, segue da proposição 1.f.1, item 4, que dado $\varepsilon=1$, existe $n$

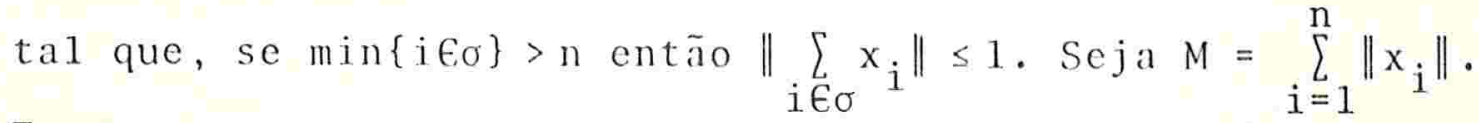
Temos que:

$$
\begin{aligned}
\left\|x_{n_{1}}+x_{n_{2}}+\ldots+x_{n_{\ell}}\right\| & +\left\|x_{1}+x_{2}+\ldots+x_{m}\right\| \leq\left\|\sum_{n_{i} \leq n} x_{n_{i}}\right\|+\left\|\sum_{n_{i}>n} x_{n_{i}}\right\|+ \\
& +\left\|\sum_{i \leq n} x_{i}\right\|+\left\|\sum_{i>n}^{m} x_{i}\right\| \leq 2 \sum_{i \leq n}\left\|x_{i}\right\|+2 \leq 2(M+1) .
\end{aligned}
$$

Então, para toda $\delta € \Delta$, vale que $\left\|\sum_{i=1}^{k}\left[f\left(t_{i}\right)-f\left(s_{i}\right)\right]\right\| \leq 2(M+1)$. Resulta que $\omega[f] \leq 2(M+1)$, ou seja, $f \in B W([0,1], X)$. Afirmamos que $f \notin B V([0,1], X)$. De fato, seja

$$
\mathrm{d}=\left\{0, \frac{1}{2}, \frac{2}{3}, \frac{3}{4}, \ldots, \frac{\mathrm{n}}{\mathrm{n}+1}, 1\right\} \text {. }
$$

Calculando $\sum_{i=1}^{|d|}\left\|f\left(t_{i}\right)-f\left(t_{i-1}\right)\right\|$, tem-se imediatamente que

$$
\sum_{i=1}^{|d|}\left\|f\left(t_{i}\right)-f\left(t_{i-1}\right)\right\| \geq \sum_{i=2}^{n}\left\|x_{i}\right\|
$$


e, como a sêrie $\sum_{i=1}^{\infty} x_{i}$, não ë absolutamente convergente, vem que $V[f]=+\infty$, e $f \notin B V[0,1], X)$.

Para o caso do intervalo $[a, b]$, não existe dificuldade adicional e deixamos a cargo do leitor esta constatação. CQD

COROLARIO 1.g.2 - Seja X um espaço de Banach. São equivalentes:

1) $\operatorname{BV}([a, b], x) \notin B W([a, b], x)$.

2) X tem dimensão infinita.

DEMONSTRACĀO - Imediata, a partir do teorema $1 . \mathrm{g} .1$.

$\underline{D E F I N I C \bar{A} O} 1 . g \cdot 3$ - Se X ë um espaço de Banach, diremos que $f:[a, b] \longrightarrow x$ é localmente de variação limitada forte em $c \in[a, b]$ se existe, um intervalo $[d, f]$ contendo $c$ no seu interior tal que $f$ restrita a $[d, f]$ pertence ao espaço

$$
B V([d, f], x) \text {. }
$$

Não demonstraremos a proposição que se segue para não alongar em demasia este capítulo introdutörio. E um trabalho em conjunto da Professora Carmen Silvia Cardassi e deste autor.

$\underline{\text { PROPOSIC } \bar{A} O} 1 . g \cdot 4$ - Se X ë um espaço de Banach, são equivalentes:

1) X tem dimensão infinita.

2) $\operatorname{BV}([a, b], X) \& B W([a, b], X)$. 
3) Existe $f \in B \omega([a, b], X)$ que não é localmente de variação limitada forte em nenhum ponto de [a,b].

4) Existe $f \in B(\omega([a, b], X)$ tal que, para todo $M>0$, existe uma partição $d=\left\{a=t_{0}<t_{1} \cdots t_{n}=b\right\}$ tal que

$$
\sum_{i=1}^{d} w_{\left[t_{i-1}, t_{i}\right]^{[f]} \geq M,}
$$

(então $f \notin B V([a, b], x))$.

$\underline{\text { OBSERVAÇÄO }} \underline{1 . g .5}$ - 0 teorema $1 . g \cdot 1$ não se encontra nos 1ivros que tratam das funções de variação fraca limitada, mas nosso orientador nos chamou a atenção que se trata de um resultado conhecido. Veja, por exemp1o: Thorp,B.L.D., on the equivalence of certain notions of bounded variation, Journal of the London Mathematical Society, 43 (170): 247-252, 1968. 
CAPITULO 2

NOÇŌES SOBRE GEOMETRIA DE ESPAÇOS DE BANACH

Neste capitulo abordaremos rapidamente as noçöes que usaremos na solução dos problemas citados no capítulo 1. No item a, deste capítulo, estão os conceitos usados na discussão e solução dos problemas $[\mathrm{P}-2],[\mathrm{P}-5],[\mathrm{P}-6],[\mathrm{P}-7],[\mathrm{P}-8]$ e suas consequências.

No item b, estão os conceitos usados na discussão e resolução do problema 3 .

2.a - ESPAÇOS DE BANACH QUE CONTEM SUBESPAÇO ISOMORFO A $C_{0}(\mathbb{N})$. ESPAÇOS REFLEXIVOS E ESPAÇOS FRACAMENTE SEQUENCIALMENTE COMPLETOS

DEFINICĀO $2 \cdot a \cdot 1$ - Uma sequência $\left\{x_{n}\right\}_{n=1}^{\infty}$ em um espaço de Banach $x$ é dita uma base de Schauder de $X$ se, para todo vetor $x$ pertencente a $X$, existe uma ünica sequência de escalares $\left\{a_{n}\right\}_{n=1}^{\infty}$ tal que $x=\sum_{n=1}^{\infty} a_{n} x_{n}$. Se $\left\{x_{n}\right\}_{n=1}^{\infty} \vec{e}$ uma base de Schauder de $X$ e para todo $x \in X$, a sërie $x=\sum_{n=1}^{\infty} a_{n} x_{n}$ é incondicionalmente convergente, (veja definição 1.f.2) diremos que 
$\left\{x_{n}\right\}_{n=1}^{\infty} \hat{e}$ uma base incondicional de $x$.

$\underline{\text { DEFINIÇÄO }} \underline{2 \cdot a \cdot 2}$ - Uma sequência $\left\{x_{n}\right\}_{n=1}^{\infty}$ que ébase de Schauder do fecho do subespaço gerado por ela é dita uma sequência básica.

$\underline{\text { PROPOSICĀO }} \underline{2 . a .3}$ - Seja X um espaço de Banach com base de Schauder $\left\{x_{n}\right\}_{n=1}^{\infty}$.

1) Para todo $x=\sum_{n=1}^{\infty} a_{n} x_{n}$, seja $\|x\|=\sup _{n \in \mathbb{N}}\left\|\sum_{i=1}^{n} a_{i} x_{i}\right\|$. Então
|| é uma norma.

2) A mesma ||$\|\quad\|$ é equivalente à norma $\|$ \| do espaço X.

3) As projeções $P_{n}: x \rightarrow x$, definidas por

$$
P_{n}\left(\sum_{i=1}^{\infty} a_{i} x_{i}\right)=\sum_{i=1}^{n} a_{i} x_{i}
$$

são funções lineares, contínuas e $K=\sup _{n \in \mathbb{N}}\left\|P_{n}\right\|<\infty$.

DEMONSTRAÇÄO - 1) Imediata .

2) Em primeiro lugar, observemos que um cálculo mostra que $(X,|\|\mid\|)$ è um espaço de Banach (veja, se necessärio,[S-I], pảgina 18, proposição 3.1). Por outro 1ado,

$$
\|x\|=\sup _{n \in \mathbb{N}}\left\|\sum_{i=1}^{n} a_{i} x_{i}\right\| \geq\left\|\lim _{n \rightarrow \infty}\right\| \sum_{i=1}^{n} a_{i} x_{i}\|=\| x \| .
$$

Logo $\|x\| \leq\|\| x \|$ e a função identidade $I:(X,\|\| \|) \rightarrow(x,\|\|)$ é contínua. Pelo teorema da aplicação aberta, I e bicontínua, e existem constantes $K>0$ e $K^{\prime}>0$, tais que

$$
K^{\prime}\|x\| \leq\|x\| \leq K\|x\|
$$


Segue que \|\| é equivalente a \|\|$\|$.

3) $\left\|P_{n}(x)\right\|=\left\|\sum_{i=1}^{n} a_{i} x_{i}\right\| \leq \sup _{n \in \mathbb{N}}\left\|\sum_{i=1}^{n} a_{i} x_{i}\right\|=\|x\| \leq k\|x\|$. Assim, $\left\|P_{n}\right\| \leq K$, para todo $n \in \mathbb{N}$, donde concluimos que as $P_{n}$ säo continuas e $\sup _{n \in \mathbb{N}}\left\|P_{n}\right\| \leq K$.

DEFINICĀO $2 . a \cdot 4$ - Dado um espaço de Banach $x$ com base de Schauder $\left\{x_{i}\right\}_{i=1}^{\infty}$, sejam $P_{n}$ as projeções da proposição 2.a.3. A constante $K=\sup _{n \in \mathbb{N}}\left\|P_{n}\right\| \vec{e}$ denominada constante da base $\left\{x_{i}\right\}_{i=1}^{\infty}$. Se $K=1$, então $\left\{x_{i}\right\}_{i=1}^{\infty}$ è dita base monôtona.

PROPOSICĀO 2.a.5 - Seja X um espaço de Banach:

1) Uma base $\left\{x_{i}\right\}_{i=1}^{\infty}$ é monötona se, e sô se para toda se-quência de escalares $\left\{a_{i}\right\}_{i=1}^{\infty}$ a sequência de números

$$
\left\{\left\|\sum_{i=1}^{n} a_{i} x_{i}\right\|\right\}_{n=1}^{\infty}
$$

é não decrescente.

2) Toda base $\left\{x_{i}\right\}_{i=1}^{\infty} \vec{e}$ monótona com relação à norma

$$
\|x\|=\sup _{n \in \mathbb{N}}\left\|P_{n}(x)\right\| \text {. }
$$

DEMONSTRAÇĀO - 1) Seja $\left\{x_{i}\right\}_{i=1}^{\infty}$ uma base monötona. Se $m \geq n$, vale que

$$
\left\|\sum_{i=1}^{n} a_{i} x_{i}\right\|=\left\|P_{n}\left(\sum_{i=1}^{m} a_{i} x_{i}\right)\right\| \leq\left\|P_{n}\right\|\left\|\sum_{i=1}^{m} a_{i} x_{i}\right\|=\left\|\sum_{i=1}^{m} a_{i} x_{i}\right\| .
$$

Logo, se $m \geq n,\left\|\sum_{i=1}^{n} a_{i} x_{i}\right\| \leq\left\|\sum_{i=1}^{m} a_{i} x_{i}\right\|$. 
$\Longleftrightarrow$ E verdade que $\left\|P_{n}(x)\right\|=\left\|\sum_{i=1}^{n} a_{i} x_{i}\right\|$ e, pela monotonicidade, temos

$$
\left\|\sum_{i=1}^{n} a_{i} x_{i}\right\| \leq\left\|\sum_{i=1}^{m} a_{i} x_{i}\right\| \leq\|x\| \text { se } m \geq n .
$$

Logo, $\left\|P_{n}(x)\right\| \leq\|x\|$, para todo $x \in X$, isto $\bar{e}\left\|P_{n}\right\| \leq 1$. Concluimos que $\left\|P_{n}\right\|=1$, para todo $n \in \mathbb{N}$, e $\sup _{n \in \mathbb{N}}\left\|P_{n}\right\|=1$.

2) Fixado um indice $n_{0}$, temos que, para todo $x \in X$,

$$
\left\|P_{n_{0}}(x)\right\|=\sup _{n \leq n}\left\|\sum_{i=1}^{n} a_{i} x_{i}\right\| \leq \sup _{n \in \mathbb{N}}\left\|\sum_{i=1}^{n} a_{i} x_{i}\right\|=\|x\| .
$$

Logo, como $\mathrm{P}_{\mathrm{n}_{0}}$ é uma projeção, $\left\|\mathrm{P}_{\mathrm{n}_{0}}\right\| \geq \geq 1$, e vem que

$$
\left\|\mathrm{P}_{\mathrm{n}_{0}}\right\| \mid=1
$$

$\underline{\text { PROPOSIÇĀO }} \underline{2 \cdot a \cdot 6}-$ Seja $\left\{x_{n}\right\}_{n=1}^{\infty}$ una sequência de vetores num espaço de Banach $x$. Então $\left\{x_{n}\right\}_{n=1}^{\infty}$ é uma base de Schauder de $X$ se, e somente se, as três condições abaixo valem simultaneamente:

1) $x_{n} \neq 0$, para todo $n$ natura 1 .

2) Existe uma constante $K>0$, tal que, para toda escotha de escalares $\left\{a_{i}\right\}_{i=1}^{\infty}$ e inteiros $n \leq m$ temos

$$
\left\|\sum_{i=1}^{n} a_{i} x_{i}\right\| \leq k\left\|\sum_{i=1}^{m} a_{i} x_{i}\right\|
$$

3) 0 fecho do espaço gerado por $\left\{x_{n}\right\}_{n=1}^{\infty}$ é $x$.

Mais ainda, 1) e 2) dão uma condição necessäria e suficien- 
te para que $\left\{x_{n}\right\}_{n=1}^{\infty}$ seja uma sequência bảsica.

DEMONSTRAÇĀO - Necessidade:

1) Se $x_{n}=0$ temos $0=x_{n}$ e $0=\sum_{i=1}^{\infty} 0 x_{i}$, o que contraria a unicidade de representação.

2) Vale $\left\|P_{n}\left(\sum_{i=1}^{m} a_{i} x_{i}\right)\right\| \leq\left\|P_{n}\right\|\left\|\sum_{i=1}^{m} a_{i} x_{i}\right\|$. Logo,

$$
\left\|\sum_{i=1}^{n} a_{i} x_{i}\right\| \leq k\left\|\sum_{i=1}^{m} a_{i} x_{i}\right\|
$$

onde $K=\sup _{n \in \mathbb{N}}\left\|P_{n}\right\|$ (vide $2 \cdot a \cdot 3$, item 3 ).

3) E decorrência imediata de definição de base.

Suficiência: a) Unicidade do desenvolvimento. Se

$$
\sum_{i=1}^{\infty} a_{i} x_{i}=\sum_{i=1}^{\infty} b_{i} x_{i} \text { então } \sum_{i=1}^{\infty}\left(a_{i}-b_{i}\right) x_{i}=0
$$

ou, se denotarmos $a_{i}-b_{i}=c_{i}$, vem que $\sum_{i=1}^{\infty} c_{i} x_{i}=0$. Se $c_{i_{0}} \neq 0$ para algum $i_{0}$, como $x_{i_{0}} \neq 0$ (hipötese), vem que

$$
\begin{aligned}
0<\left\|c_{i_{0}} x_{i_{0}}\right\| & =\left\|\sum_{i=1}^{i_{0}} c_{i} x_{i}-\sum_{i=1}^{i_{0}^{-1}} c_{i} x_{i}\right\| \leq\left\|\sum_{i=1}^{i_{0}} c_{i} x_{i}\right\|+\left\|\sum_{i=1}^{i_{0}-1} c_{i} x_{i}\right\| \leq \\
& \leq K\left\|\sum_{i=1}^{n} c_{i} x_{i}\right\|+k\left\|\sum_{i=1}^{n} c_{i} x_{i}\right\| \leq 2 K\left\|\sum_{i=1}^{n} c_{i} x_{i}\right\|,
\end{aligned}
$$

para todo $n \in \mathbb{N}, n>i_{0}$. Logo, $\left\|\sum_{i=1}^{\infty} c_{i} x_{i}\right\|>0$, contradição a $\sum_{i=1}^{\infty} c_{i} x_{i}=0$. Concluímos que $c_{i}=0$, para todo $i \in \mathbb{N}$ e portanto $\mathrm{a}_{i}=\mathrm{b}_{i}$. 
b) Um cálculo simples mostra (usando o item 2) que o espaço dos elementos da forma $\sum_{n=1}^{\infty} a_{n} x_{n}$ é um subespaço $F$ fechado de $X$ (veja [S-I], pägina 18, proposição 3.1). Dessa forma, 1) e 2) implicam que $\left\{x_{i}\right\}_{i=1}^{\infty} \vec{e}$ uma sequência básica.Por outro lado, 3) implica que $F=X$, o que termina a demonstração.

CQD

DEFINIÇĀO $2 . a \cdot 7$ - Uma base de Schauder $\left\{x_{n}\right\}_{n=1}^{\infty}$ de um espaço de Banach $x \vec{e}$ dita normalizada se $\left\|x_{n}\right\|=1$, para todo $n$.

$\underline{\text { PROPOSICĀO }} \underline{2 . a .8}$ - Se $x$ é um espaço de Banach e $\left\{x_{n}\right\}_{n=1}^{\infty}$ uma base de $x$ então $\left\{\frac{x_{n}}{\left\|x_{n}\right\|}\right\}_{n=1}^{\infty}$ é uma base normalizada de $x$.

DEMONSTRAÇĀO - Obvia.

2.a.9 EXEMPLOS E OBSERVAÇĀO - Os vetores $e_{n}=(00 \ldots 100 \ldots 0)$ formam uma base monótona e normalizada de $C_{0}(\mathbb{N})$ e $\ell_{p}, 1 \leq p<\infty$. E claro que, para um espaço de Banach ter base de Schauder $\left\{x_{i}\right\}_{i=1}^{\infty} \vec{e}$ necessário que ele seja separävel, pois o conjunto $\left\{\sum_{i=1}^{n} r_{i} x_{i} \mid r_{i} \in \mathbb{Q}\right\}$ é enumerâvel e denso no espaço. Aquestão posta por Banach: "Todo espaço de Banach separável tem uma base?" foi respondida negativamente por P. Enflo (veja [E-P]). No entanto, a tếcnica de trabalhar com uma base de Schauder de um subespaço é bastante útil na solução e discussões dos problemas propostos no capítulo 1. Este procedimento ê possível graças ao resultado conhecido: "Todo espaço de Banach de dimensão infinita contém uma sequênciabásica", e sua variante, que envolve sequências que convergem 
fracamente a zero, mas não contém nenhuma subsequência que converge em norma a zero. Vejamos estes resultados:

LEMA $\underline{2 . a \cdot 10}$ - Seja X um espaço de Banach de dimensão infinita. Seja $B C X$ um subespaço vetorial de $X$ de dimensão finita $e \quad \varepsilon>0$.

a) Existe $x \in X$ com $\|x\|=1$ tal que, para todo $y \in B$ e todo escalar $\lambda$ temos, $\|y\| \leq(1+\varepsilon)\|y+\lambda x\|$.

b) Se $\left\{x_{n}\right\}_{n=1}^{\infty}$ è uma sequência tal que 1 im inf $\left\|x_{n}\right\|>0$ e $x_{n} \underset{n \rightarrow \infty}{\stackrel{\text { fracamente }}{n \rightarrow \infty}} 0$ então existe uma subsequência

$$
y_{n_{k}}=\frac{x_{n_{k}}}{\pi x_{n_{k}} \|}
$$

e existe $x \in\left\{y_{n_{k}}\right\}_{k=1}^{\infty}$ tal que $\|y\| \leq(1+\varepsilon)\|y+\lambda x\|$, para todo $y \in B$, para todo $\lambda$ escalar.

DEMONSTRAÇĀO - E claro que, se $\|y\| \leq(1+\varepsilon)\|y+\lambda x\|$, para $0<\varepsilon<1$, então $\|y\| \leq(1+\varepsilon)\|y+\lambda x\|$ para $\varepsilon \geq 1$, assim vamos nos restringir ao caso $0<\varepsilon<1$. E tambëm claro que se $\|y\| \leq(1+\varepsilon)\|y+\lambda x\|$ ë verdadeira para $\|y\|=1$, taunbém é verdadeira para todo $y$ pertencente a $B$, pois, se

$$
\left\|\frac{y}{\|y\|}\right\| \leq(1+\varepsilon)\left\|\frac{y}{\|y\|}+\lambda x\right\|
$$

vale para todo $\lambda$ escalar, então, $\|y\| \leq(1+\varepsilon)\left\|y+\lambda_{1} x\right\|$ vale para todo $\lambda_{1}$ escalar.

Dessa forma, consideraremos apenas $\|y\|=1$.

Pela compacidade da bola unitäria de $B$, dado $\varepsilon>0$, 
existem elementos $y_{i}, 1 \leq i \leq m, \operatorname{com}\left\|y_{i}\right\|=1$ e tais que, para todo $y \in B$, com $\|y\|=1$ existe $1 \leq i \leq m$, tal que $\left\|y-y_{i}\right\|<\frac{\varepsilon}{2}$. Pelo teorema de Hahn-Banach, podemos tomar $y_{i}^{\prime} \in X^{\prime}, 1 \leq i \leq m$ tais que $\left\|y_{i}^{\prime}\right\|=1$ e $y_{i}^{\prime}\left(y_{i}\right)=1$.

a) Como a dimensão de $x$ é infinita, existe $x \in x,\|x\|=1$, tal que $y_{i}^{\prime}(x)=0,1 \leq i \leq m$. Assim, dado $y \in B,\|y\|=1$, se escolhermos $y_{i}$ convenientemente, vem que, para todo escalar $\lambda$ temos

$$
\|y+\lambda x\|=\left\|y_{i}+\lambda x-\left(y_{i}-y\right)\right\| \geq\left\|y_{i}+\lambda x\right\|-\left\|y-y_{i}\right\| \geq\left\|y_{i}+\lambda x\right\|-\frac{\varepsilon}{2} .
$$

Ainda pelo teorema de Hahn-Banach, $\left|y_{i}^{\prime}\left(y_{i}-\lambda x\right)\right| \leq\left\|y_{i}+\lambda x\right\|$ e podemos concluir, da desigualdade acima, que

$$
\|y+\lambda x\| \geq\left|y_{i}^{\prime}\left(y_{i}+\lambda x\right)\right|-\frac{\varepsilon}{2}=\left|y_{i}^{\prime}\left(y_{i}\right)+\lambda y_{i}^{\prime}(x)\right|-\frac{\varepsilon}{2}=1-\frac{\varepsilon}{2} \geq \frac{1}{1+\varepsilon}=\frac{\|y\|}{1+\varepsilon} .
$$

Daí vem que $\|y\| \leq(1+\varepsilon)\|y+\lambda x\|$.

b) Como $x_{n} \underset{n \rightarrow \infty}{\text { fracamente }} 0$, existe $x \in\left\{\frac{x_{n}}{\left\|x_{n}\right\|}\right\}_{n=1}^{\infty}$ tal que

$$
\left|y_{i}^{\prime}(x)\right|<\frac{\varepsilon}{4} \text {, para todo } 1 \leq i \leq m .
$$

Para todo $|\lambda| \geq 2$, temos que $\|y+\lambda x\| \geq\|\lambda x\|-\|y\| \geq 2-1 \geq 1=\|y\|$, is to $\bar{e},\|y\| \leq\|y+\lambda x\|$.

Para $|\lambda|<2$, retomando parte das desigualdades docaso a) obtemos:

$$
\begin{aligned}
\|y+\lambda x\| & \geq\left\|y_{i}+\lambda x\right\|-\frac{\varepsilon}{2} \geq\left|y_{i}^{\prime}\left(y_{i}+\lambda x\right)\right|-\frac{\varepsilon}{2}=\left|y_{i}^{\prime}\left(y_{i}\right)+\lambda y_{i}^{\prime}(x)\right|-\frac{\varepsilon}{2} \geq \\
& \geq 1-\left|\lambda y_{i}^{\prime}(x)\right|-\frac{\varepsilon}{2}>1-2 \cdot \frac{\varepsilon}{4}-\frac{\varepsilon}{2}=1-\varepsilon=(1-\varepsilon)\|y\|,
\end{aligned}
$$


e portanto, $\|y\| \leq \frac{1}{1-\varepsilon}\|y+\lambda x\|$.

$$
\begin{aligned}
& \text { Dessa forma, para todo escalar } \lambda \text { temos } \\
& \qquad\|y\| \leq\|y+\lambda x\| \text { ou }\|y\| \leq \frac{1}{1-\varepsilon}\|y+\lambda x\|,
\end{aligned}
$$

donde se conclui que $\|y\| \leq \frac{1}{1-\varepsilon}\|y+\lambda x\|$, para todo escalar $\lambda$. (Sendo $\varepsilon$ arbiträrio, ë verdade que

$$
\left.\|y\| \leq(1+\delta)\|y+\lambda x\|, \operatorname{com} 1+\delta=\frac{1}{1-\varepsilon} \cdot\right) \quad \text { CQD }
$$

TEOREMA 2.a.11 - 1) Todo espaço de Banach de dimensäo infinita contêm uma sequência bảsica.

2) $\operatorname{Se}\left\{x_{n}\right\}_{n=1}^{\infty}$ é uma sequência de vetores em $x$ tal que

$$
1 \text { im inf }\left\|x_{n}\right\|>0 \text { e } x_{n} \frac{\text { fracamente }}{n \rightarrow \infty} 0
$$

então $\left\{x_{n}\right\}_{n=1}^{\infty}$ possui uma subsequência $\left\{x_{n_{k}}\right\}_{k=1}^{\infty}$ que è uma sequência bâsica.

$\frac{\text { DEMONSTRACĀA }}{\infty}$ - 1) Seja $\varepsilon>0$ e $\left\{\varepsilon_{n}\right\}_{n=1}^{\infty}$ números positivos tais que $\prod_{n=1}\left(1+\varepsilon_{n}\right) \leq 1+\varepsilon$.

Seja $x_{1}$ um elemento de $X$ de norma 1 e $B_{1}$ o espaço gerado por $x_{1}$. Por a) do lema 2 .a.10, existe $x_{2} \in X,\left\|x_{2}\right\|=1$, tal que $\|y\| \leq\left(1+\varepsilon_{1}\right)\left\|y+\lambda x_{2}\right\|$, para todo $y$ de $B_{1}$ e todo esca$\operatorname{lar} \lambda$. Por indução construïmos $\left\{x_{n}\right\}_{n=1}^{\infty}$ tal que

$$
\|y\| \leq\left(1+\varepsilon_{n}\right)\left\|y+\lambda x_{n+1}\right\|
$$

para todo y no espaço $B_{n}$ gerado por $\left\{x_{1} \ldots x_{n}\right\}$, e $\lambda$ escalar qualquer. A sequência $\left\{x_{n}\right\}_{n=1}^{\infty}$ è básica com constante de ba- 
se menor ou igual a $1+\varepsilon$. De fato: todos os $x_{i}$ são diferentes de zero, pela forma com que foram escolhidos $(\|x\|=1$, para todo i). Alëm disso,

$$
\left\|\sum_{i=1}^{n} a_{i} x_{i}\right\| \leq\left(1+\varepsilon_{n}\right)\left\|\sum_{i=1}^{n+1} a_{i} x_{i}\right\| \leq\left(1+\varepsilon_{n}\right)\left(1+\varepsilon_{n+1}\right) \ldots\left(1+\varepsilon_{n+p}\right)\left\|\sum_{i=1}^{n+p+1} a_{i} x_{i}\right\| .
$$

Portanto, se $m \geq n$, virä

$$
\left\|\sum_{i=1}^{n} a_{i} x_{i}\right\| \leq(1+\varepsilon)\left\|\sum_{i=1}^{m} a_{i} x_{i}\right\|
$$

Verificamos então 1) e 2) da proposição 2.a.6, o que demonstra a parte 1) do teorema.

2) $\hat{E}$ a mesma do item 1), tomando-se o cuidado de escother $x_{1} \in\left\{\frac{x_{n}}{\left\|x_{n}\right\|}\right\}_{n=1}^{\infty}$, sendo $B_{1}$ o espaço gerado por $x_{1}$, e aplicar, na indução, o lema $2 . a .10$, item b) CQD

TEOREMA 2 .a.12 - Se X ẻ um espaço de Banach, combase incondicional e não contém subespaço isomorfo a $C_{0}(\mathbb{N})$ ou a $\ell_{1}(\mathbb{N})$ então x é reflexivo.

DEMONSTRACĀO - Veja $[R-C-2]$.

O teorema que se segue $\vec{e}$ devido a Bessaga-Pelczynski (veja [B-P]) e ser-nos-ā ütil na solução dos problemas $[\mathrm{P}-2],[\mathrm{P}-5]$ e consequências.

TEOREMA 2.a.13 - Seja X um espaço de Banach. São equivalentes as propriedades:

1) $X$ contém um subespaço isomorfo a $C_{0}(\mathbb{N})$. 
2) Existe uma sequência $\left\{x_{n}\right\}_{n=1}^{\infty}$ em $x$ tal que $\sum_{n=1}^{\infty} x_{n}$ não converge mas, para todo $x^{\prime} \in X^{\prime}$,

$$
\sum_{n=1}^{\infty}\left|x^{\prime}\left(x_{n}\right)\right| \text { converge. }
$$

DEMONSTRAC $\vec{A} O-1) \Longrightarrow 2$ ) Suponhamos que $Y \vec{e}$ subespaço de $X$ isomorfo a $C_{0}(\mathbb{N})$, atravës do isomorfismo J:C $C_{0}(\mathbb{N}) \rightarrow Y$. Para cada neN, tomemos $e_{n}=(00 \ldots 100 \ldots)$. Sendo $\left\|e_{n}\right\|=1$, temos que $\left\|J\left(e_{n}\right)\right\| \geq \delta>0$, e portanto, a sërie $\sum_{n=1}^{\infty} J\left(e_{n}\right)$ não converge. Por outro 1ado, como $\sum_{n=1}^{\infty}\left|x^{\prime}\left(e_{n}\right)\right|$ converge para todo $x^{\prime} \varepsilon_{1}(\mathbb{N})$. então $\sum_{n=1}^{\infty}\left|y^{\prime}\left(J\left(e_{n}\right)\right)\right| \begin{aligned} & n=1 \\ & \text { converge para todo } y^{\prime} \text { pertencente a } Y^{\prime}\end{aligned}$ ( ja que $y^{\prime}$ oJE $\left.\left[C_{0}(\mathbb{N})\right]^{\prime}=\ell_{1}(\mathbb{N})\right)$ e consequentemente, para todo $x^{\prime} \in X^{\prime}$.

2) $\Longrightarrow 1)$ Suponhamos que, em $x$ exista uma sêrie $\sum_{n=1}^{\infty} x_{n} d i-$ vergente tal que $\sum_{n=1}^{\infty}\left|x^{\prime}\left(x_{n}\right)\right|<\infty$, para todo $x^{\prime}$ pertencente a X' Então, pelo princípio da limitação uniforme, existe uma constante $M>0$ tal que $\sum_{n=1}^{\infty}\left|x^{\prime}\left(x_{n}\right)\right| \leq M\left\|x^{\prime}\right\|$ (1).

Como $\sum_{n=1}^{\infty} x_{n}$ diverge, existe $\varepsilon_{0}>0$ e inteiros $p_{1}<q_{1}<$ $<p_{2}<q_{2}<\ldots$ tais que

$$
\left\|\sum_{n=p_{k}}^{q_{k}} x_{n}\right\| \geq \varepsilon_{0}
$$

para todo $k$ natural.

Tomemos $y_{k}=\sum_{n=p_{k}}^{q_{k}} x_{n} ;$ assim, $\left\|y_{k}\right\| \geq \varepsilon_{0}$, para todo $k$ natura1.

Como $\sum_{n=1}^{\infty}\left|x^{\prime}\left(x_{n}\right)\right|<\infty$, para todo $x^{\prime}$ pertencente a $x^{\prime}$, vem que 
$\sum_{k=1}^{\infty}\left|x^{\prime}\left(y_{k}\right)\right|<\infty$, para todo $x^{\prime}$ pertencente a $x^{\prime}$, e, em consequência, $\left|x^{\prime}\left(y_{k}\right)\right| \underset{k \rightarrow \infty}{\longrightarrow} 0$, ou scja, $y_{k} \frac{\text { fracamente }}{k \rightarrow \infty} 0$ (3). Usando (2) e (3) podemos aplicar 2) do teorema 2.a.11: $\left\{y_{k}\right\}_{k=1}^{\infty}$ possui uma subsequência, que continuaremos denotando por $\left\{y_{k}\right\}_{k=1}^{\infty}$ que é uma sequência bảsica em $x$, com constante básica que denotaremos por $K$.

Tomemos uma sequência de escalares $\left\{a_{i}\right\}_{i=1}^{m}$. Se $i \leq m$, temos:

$$
\left|a_{i}\right|\left\|y_{i}\right\|=\left\|\sum_{j=1}^{i} a_{j} y_{j}-\sum_{j=1}^{i-1} a_{j} y_{j}\right\| \leq\left\|\sum_{j=1}^{i} a_{j} y_{j}\right\|+\left\|\sum_{j=1}^{i-1} a_{j} y_{j}\right\| \leq 2 K\left\|\sum_{j=1}^{m} a_{i} y_{i}\right\|
$$

Logo ,

$$
\frac{\max _{1 \leq \mathrm{m}}\left|\mathrm{a}_{i}\right| \times \varepsilon_{0}}{2 \mathrm{~K}} \leq\left\|\sum_{i=1}^{m} \mathrm{a}_{i} y_{i}\right\|
$$

Por outro lado, pelo teorema de Hahn-Banach e por (1), temse que:

$$
\begin{aligned}
& \left\|\sum_{j=1}^{m} a_{j} y_{j}\right\|=\sup _{\left\|x^{\prime}\right\| \leq 1}\left\{\left|\sum_{j=1}^{m} a_{j} x^{\prime}\left(y_{j}\right)\right|\right\} \leq \sup _{\left\|x^{\prime}\right\| \leq 1}\left\{\sum_{j=1}^{m}\left|a_{j}\right|\left|x^{\prime}\left(y_{j}\right)\right|\right\} \leq \\
& \leq \max _{1 \leq j \leq m}\left|a_{j}\right| \sup _{\left\|x^{\prime}\right\| \leq 1}\left\{\sum_{j=1}^{m}\left|x^{\prime}\left(y_{j}\right)\right|\right\} \leq\left(\max _{1 \leq j \leq m}\left|a_{j}\right|\right) M\left\|x^{\prime}\right\| \leq M \max _{1 \leq j \leq m}\left|a_{j}\right|,
\end{aligned}
$$

e portanto,

$$
\left\|\sum_{j=1}^{m} a_{j} y_{j}\right\| \leq M \max _{1 \leq j \leq m}\left|a_{j}\right|(5) \text {. }
$$

De (4) e (5), obtemos: 


$$
\frac{\varepsilon_{0}}{2 K} \max _{1 \leq i \leq m}\left|a_{i}\right| \leq\left\|\sum_{i=1}^{m} a_{i} y_{i}\right\| \leq M \max _{1 \leq i \leq m}\left|a_{i}\right|
$$

e é decorrência imediata destas desigualdades que a função linear $J: C_{0}(\mathbb{N}) \longrightarrow X$ (obtida tomando-se $J\left(e_{i}\right)=y_{i}$, para todo $i \in \mathbb{N})$ é um isomorfismo entre $C_{0}(\mathbb{N})$ e o fecho do espaço gerado por $\left\{y_{i}\right\}_{i=1}^{\infty}$.

COROLÁRIO 2.a.14 - Seja X um espaço de Banach. São equivalentes as propriedades:

1) $X$ contëm subespaço isomorfo a $C_{0}(\mathbb{N})$.

2) Existe $\delta>0$ e uma sequência de vetores $\left\{x_{n}\right\}_{n=1}^{\infty}$ com $\left\|x_{n}\right\| \geq \delta$ e tal que

$$
\sup _{\substack{\mathrm{K} \in \mathbb{N} \\\left|\lambda_{\mathrm{N}}\right| \leq 1}}\left\|\sum_{n=1}^{K} \lambda_{n} x_{n}\right\| \leq M<\infty
$$

DEMONSTRAC $\bar{A} O-1) \Longrightarrow 2$ ) Considere o isomorfismo

$$
\mathrm{J}: \mathrm{C}_{0}(\mathbb{N}) \rightarrow \mathrm{Y} \subset \mathrm{X}
$$

e seja $e_{n}=(0,0 \ldots 0,1,0,0 \ldots)$. Se considerarmos $x_{n}=J\left(e_{n}\right)$ então existe $\delta>0$ tal que $\left\|J\left(e_{n}\right)\right\| \geq \delta=\left\|J^{-1}\right\|^{-1}$, para todo $n$, $\mathrm{e}$

$$
\sup _{\left|\lambda_{n}\right| \leq 1}\left\|\sum_{n=1}^{K} \lambda_{n} J\left(e_{n}\right)\right\| \leq\|J\| \sup _{\left|\lambda_{n}\right| \leq 1}\left\|\sum_{n=1}^{K} \lambda_{n} e_{n}\right\| \leq\|J\| \cdot 1 .
$$

KEN KEN

2) $\Longrightarrow 1$ 1) Considere a sërie $\sum_{n=1}^{\infty} x_{n}$. Ë imediato que ela não 
converge pois $\left\|x_{n}\right\| \geq \delta$, para todo $n$. Por outro lado, se considerarmos $\lambda_{n}=\sin x^{\prime}\left(x_{n}\right)$ teremos

$$
\begin{aligned}
& \sum_{n=1}^{K}\left|x^{\prime}\left(x_{n}\right)\right|=\sum_{n=1}^{K} \lambda_{n} x^{\prime}\left(x_{n}\right)=x^{\prime}\left(\sum_{n=1}^{K} \lambda_{n} x_{n}\right)= \\
& =\left|x^{\prime}\left(\sum_{n=1}^{K} \lambda_{n} x_{n}\right)\right| \leq\left\|x^{\prime}\right\|\left\|\sum_{n=1}^{K} \lambda_{n} x_{n}\right\| \leq\left\|x^{\prime}\right\| M .
\end{aligned}
$$

Logo para $x^{\prime} \in X^{\prime}$ a série $\sum_{n=1}^{\infty}\left|x^{\prime}\left(x_{n}\right)\right|$ é convergente e pelo teorema 2.a.13, X contëm então subespaço isomorfo a $C_{0}(\mathbb{N})$.

No que se segue faremos um breve apanhado de resultados sobre espaços reflexivos e fracamente sequencialmente completos que precisaremos na discussão dos problemas [P-2], [P-5] e consequências.

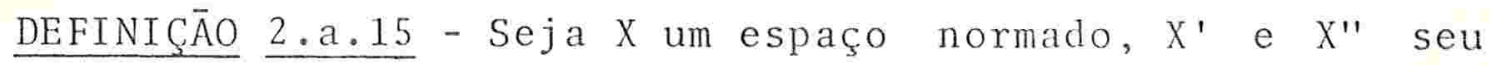
dual e bidual topolögico, respectivamente. Seja F: X $\longrightarrow X^{\prime \prime}$ dada por $F(x)=x^{\prime \prime} \operatorname{com} x^{\prime \prime}\left(x^{\prime}\right)=x^{\prime}(x)$, para todo $x \in X$ e $x^{\prime} \in X^{\prime}$, Um espaço normado $X$ é dito reflexivo se $F: X \rightarrow X^{\prime \prime}$ definida acima ë sobrejetora.

DEFINICĀO 2.a.16 - Dado um espaço normado $x$, sua topologia fraca $\sigma\left(X, X^{\prime}\right)$ é a menos fina das topologias sobre $X$ que torna continuas as aplicações $x \in X \longmapsto x^{\prime}(x)$, onde $x^{\prime} \in X^{\prime}$.

$\underline{\text { PROPOSIÇĀO }} \underline{2 . a .17}$ - 1) A topologia fraca de um espaço normado é separada.

2) Todo convexo fechado em $x$ é fracamente fechado. 
DEMONSTRACÄO - Veja $[\mathrm{H}-4]$.

O teorema que se segue è um apanhado sobre reflexividade. A equivalência de 5 , e reflexividade sera a mais usada e por este motivo damos uma demonstraçäo que supomos de nossa autoria.

TEOREMA 2.a.18 - Seja X um espaço de Banach. São equivalentes:

1) $\mathrm{x}$ è reflexivo.

2) Toda sequência limitada contêm uma subsequência fracamente convergente.

3) 0 conjunto $\overline{B_{1}(0)}=\{x \mid\|x\| \leq 1\}$ é fracamente compacto.

4) Todo funcional linear continuo tem mäximo na fronteira do conjunto $\overline{B_{1}(0)}$.

5) Toda sequência descendente $c_{1} \supset c_{2} \supset c_{3} \supset \ldots$ de convexos limitados, fechados e não vazios de $x$ têm interseção não vazia.

DEMONSTRACĀO - 1) $\Longleftrightarrow$ 2) Veja [Y], pägina 141.

1) $\Longleftrightarrow 3)$ Veja $[H-4]$, pägina 272 .

1) $\Longleftrightarrow$ 4) Veja $[R C-5]$.

Para equivalência de 5) com as outras propriedades usaremos o esquema 3 ) $\Longrightarrow 5$ ), 5) $\Longrightarrow 4$ ).

$3) \Longrightarrow$ 5) Se $c_{1} \supset c_{2} \supset c_{3} \supset \ldots$ são convexos, fechados e 1 imi- 
tados, segue de 2) da proposição 2.a.17 que são fracamente fechados e, de 3), do enunciado acima segue que a interseção é não vazia pois a bola que os contém é fracamente compacta.

$5) \Longrightarrow 4)$ (Basta provar: (não 4) $\Longrightarrow$ (não 5)). Suponhamos, por absurdo, que existisse um funcional

$$
x^{\prime} \in X^{\prime} \text { tal que não existe } x \in \overline{B_{1}(0)} \operatorname{com} x^{\prime}(x)=\left\|x^{\prime}\right\| \text {. }
$$

Considerando a sequência de convexos fechados, 1imitados e não vazios definidos por

$$
c_{n}=\left\{x^{\prime-1}\left[\left\|x^{\prime}\right\|-\frac{1}{n+1},\left\|x^{\prime}\right\|\right]\right\} \cap \overline{B_{1}(0)},
$$

é fäcil verificar que $c_{1} \supset c_{2} \supset c_{3} \supset \ldots$ e $\prod_{n=1}^{\infty} c_{n}=\emptyset$, o que contraria a hipôtese.

OBSERVAÇĀO 2.a.19 - Na realidade, no caso de um espaço normado, a propriedade 5) do teorema $2 . a .18$ é mais forte que a 4), pois esta não implica que o espaço $X$ seja completo (veja $[R-C-4])$, enquanto que é uma verificação simples mostrar que 5) implica que $X \vec{e}$ de Banach.

COROLARIO 2.a.20 - 1) Se X e Y são espaços de Banach isomorfos e $X \vec{e}$ reflexivo, então $Y \vec{e}$ reflexivo.

2) Se $Y$ é subespaço fechado de $X, e x$ è reflexivo,então Y è reflexivo.

3) $C_{0}(\mathbb{N})$ não è reflexivo. 
4) Se $X \vec{e}$ reflexivo, então $X$ não contêm nenhum subespaço isomorfo a $C_{0}(\mathbb{N})$.

DEMONSTRAÇÄO - 1) Seja $c_{1} \supset c_{2} \supset c_{3} \supset \ldots$ uma sequência de convexos, limitados, fechados e não vazios em $Y$. Se J: Y $\longrightarrow X$ $\vec{e}$ um isomorfismo entre $X$ e $Y$, $\vec{e}$ imediato que $J\left(c_{1}\right) \supset J\left(c_{2}\right) \ldots$ è uma sequência de convexos, fechados, limitados e não vazios. $\log o \prod_{i=1}^{\infty} J\left(c_{i}\right) \neq \emptyset$, e então, $J^{-1}\left(\bigcap_{i=1}^{\infty} J\left(c_{i}\right)\right)=\bigcap_{i=1}^{\infty} c_{i} \neq \varnothing$, e $Y$ $\vec{e}$ reflexivo.

2) Tomemos $c_{1} \supset c_{2} \supset c_{3} \supset \ldots$ convexos, fechados, limitados e não vazios em $Y$. Como $Y$ ë fechado, $c_{1} \supset c_{2} \supset \ldots$ sãoconvexos fechados, 1imitados e não vazios em $x ; \log 0, \bigcap_{i=1}^{\infty} c_{i} \neq \emptyset$, e segue que $Y \vec{e}$ reflexivo.

3) Para cada n natural, consideremos o conjunto

$$
c_{n}=\left\{x \in C_{0}(\mathbb{N})\|x\| \leq 1, x_{i}=1,1 \leq i \leq n\right\} .
$$

E imediato que $c_{1} \supset c_{2} \supset c_{3} \supset \ldots$ e que os $c_{i}$ são convexos, 1imitados, fechados, não vazios; para todo neN, se

$$
x=\left(x_{1}, x_{2}, \ldots, x_{n}, \ldots\right) \in \prod_{i=1}^{n} c_{i}
$$

então $x_{i}=1,1 \leq i \leq n$. Daí vem que $x \notin C_{0}(\mathbb{N}) ; \log o, \bigcap_{i=1}^{\infty} c_{i}=\varnothing$ e $C_{0}(\mathbb{N})$ não è reflexivo.

4) Consequência imediata das afirmações 1),2) e 3) CQD DEFINIÇĀO 2.a.21 - Seja X um espaço de Banach. Diremos que $x$ é fracamente sequencialmente completo se, dada uma sequên- 
cia $\left\{x_{i}\right\}_{i=1}^{\infty}$ de elementos de $x$ tal que $\lim _{i \rightarrow \infty} x^{\prime}\left(x_{i}\right)$ existe, para todo $x^{\prime} \in X^{\prime}$, então existe $x \in X$ tal que $x^{\prime}(x)=\lim _{i \rightarrow \infty} x^{\prime}\left(x_{i}\right)$, para todo $x^{\prime} \in X^{\prime}$.

$\underline{\text { PROPOSIÇĀO }} 2 . a \cdot 22$ - 1) Se X é um espaço de Banach fracamente sequencialmente completo e $Y \vec{e}$ isomorfismo a $X$, então, $Y$ $\vec{e}$ fracamente sequencialmente completo.

2) Se $X \vec{e}$ um espaço de Banach fracamente sequencialmente completo e Y é subespaço fechado de X então, Y é fracamente sequencialmente completo.

3) $C_{0}(\mathbb{N})$ não e fracamente sequencialmente completo.

4) Se $x \vec{e}$ fracamente sequencialmente completo então $x$ não contẻm nenhum subespaço isomorfo a $C_{0}(\mathbb{N})$.

DEMONSTRAC.ĀO - 1) Imediata.

2) Imediata.

3) Tomemos $x_{n}=(1,1,1, \ldots 100 \ldots)$, para todo $n \in \mathbb{N}$.

Se $x{ }^{\prime} \in l_{1}(\mathbb{N})$, dado $\varepsilon>0$ existe $n_{0}$ tal que, se $n, m \geq n_{0}$ então

$$
\left|x^{\prime}\left(x_{n}-x_{m}\right)\right|=\left|x^{\prime}(0,0,0 \ldots 1,1, \ldots 1,0,0, \ldots 0, \ldots)\right| \leq \varepsilon \text {. }
$$

Portanto $\left\{x_{n}\right\} \vec{e}$ uma sequência de Cauchy fraca em $C_{0}(\mathbb{N})$.

E imediato que $\left\{x_{n}\right\}_{n=1}^{\infty}$ não converge fracamente em. $C_{0}(\mathbb{N})$, pois converge $\sigma\left(X^{\prime \prime}, X^{\prime}\right)$ para

$$
\mathrm{e}=(1,1,1 \ldots 1 \ldots) \in \mathrm{C}_{0}(\mathbb{N})^{\prime \prime}=\ell_{\infty}(\mathbb{N}) .
$$

4) E consequência imediata das afirmações 1),2) e 3). 
PROPOSIÇĀO 2.a.23 - Todo espaço reflexivo ë fracamente sequencialmente completo.

DEMONSTRACÄO - Seja $\left\{x_{n}\right\}_{n=1}^{\infty}$ uma sequência de Cauchy fraca em $x$. Assim, dado $\varepsilon=1$, existe $n_{0}$, tal que, se $n \geq n_{0}$.

$$
\left|x^{\prime}\left(x_{n}\right)-x^{\prime}\left(x_{n_{0}}\right)\right| \leq 1 \text { ou, se } n \geq n_{0}\left|x^{\prime}\left(x_{n}\right)\right| \leq\left|x^{\prime}\left(x_{n_{0}}\right)\right|+1 \text {. }
$$

Logo, $\left|x^{\prime}\left(x_{n}\right)\right| \leq M$, para todo neN. Pelo principio da limitação uniforme, o conjunto $\left\{x_{n}\right\}_{n=1}^{\infty} \vec{e} 1$ imitado na norma de $x$. Como o espaço è reflexivo, segue do teorema 2.a.18, item 2), que existe

$$
\begin{aligned}
& x_{n_{k}} \frac{\text { Eracamente }}{\mathrm{k} \rightarrow \infty} x_{0} ; \operatorname{logo}, x_{n} \frac{\text { fracamente }}{n \rightarrow \infty} x_{0} \cdot \quad \text { CQD } \\
& \text { o teorema que segue serä ütil nas considerações que }
\end{aligned}
$$
faremos após a solução dos problemas [P-2], [P-5] e conse-quências e é devido a Odel1 e Rosenthal.

TEOREMA 2.a.24 - Seja $X$ um espaço de Banach separäve1. As seguintes propriedades são equivalentes:

1) X näo contërn subespaço isomorfo a $l_{1}(\mathbb{N})$.

2) Todo elemento de $X^{\prime \prime} \vec{e} \sigma\left(X^{\prime \prime}, X^{\prime}\right)$-limite de uma sequência de elementos de $x$.

3) O cardinal de $x^{\prime \prime} \vec{e}$ igual ao cardinal de $x$.

4) Toda sequência limitada em $X^{\prime \prime}$ tem uma subsequência $\sigma\left(X^{\prime \prime}, X^{\prime}\right)$-convergente.

DEMONSTRAÇĀO - Veja $[O-R]$ e $[R]$. 
COROLÂRIO 2.a.25 - Se X è um espaço de Banach separäve1, não reflexivo, que não contëm subespaço isomorfo a $\ell_{1}(\mathbb{N})$ então $\mathrm{X}$ não $\vec{e}$ fracamente sequencialmente completo.

DEMONSTRAÇĀO - Se X não é reflexivo então por 2) do teorema 2.a.24, existe um elemento $x^{\prime \prime} \in X^{\prime \prime}, x^{\prime \prime C X}$ e existe $\left\{x_{n}\right\}_{n=1}^{\infty} c X$, com $x_{n} \frac{\sigma\left(X^{\prime \prime}, X^{\prime}\right)}{n \rightarrow \infty} \rightarrow x^{\prime \prime}$. Assim, $\left\{x_{n}\right\}_{n=1}^{\infty}$ é uma sequência de Cauchy fraca em $x$ e não convergente em $x$ : $\log o$, $x$ não é fracamente sequencialmente completo.

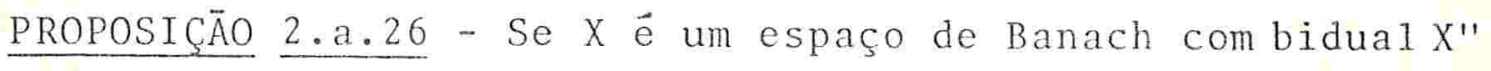
separävel, então $X$ não contëm subespaço isomorfo a $C_{0}(\mathbb{N})$ ou a $\ell_{1}(\mathbb{N})$.

$\underline{\text { DEMONSTRACĀO }}$ - 1) Se existisse um isomorfismo $T_{1}: l_{1}(\mathbb{N}) \rightarrow X$, existiria $\mathrm{T}_{2}: X^{\prime} \rightarrow\left[\mathrm{T}\left(\ell_{1}(\mathbb{N})\right)\right]^{\prime}$ definida por

$$
\mathrm{T}_{2}\left(\mathrm{x}^{\prime}\right)=\mathrm{x}^{\prime} / \mathrm{T}\left(\ell_{1}(\mathbb{N})\right)
$$

com $\mathrm{T}_{2}$ linear, $\left\|\mathrm{T}_{2}\right\|=1$, $\mathrm{T}_{2}$ sobrejetora (Teorema de Hahn-Banach). Como $X^{\prime \prime}$ é separävel concluímos que $X^{\prime}$ é separāvel. (Veja [Y], pägina 126), Portanto a função $\mathrm{T}_{2}: X^{\prime} \rightarrow \ell_{\infty}(\mathbb{N})$ é linear, contínua de um espaço separävel sobre um espaço não separävel; o que é um absurdo.

2) Se existisse $T: C_{0}(\mathbb{N}) \rightarrow X$; como anteriormente existiria $\mathrm{T}_{2}: X^{\prime} \rightarrow \ell_{1}(\mathbb{N})$ linear continua sobrejetora $\left\|\mathrm{T}_{2}\right\|=1$. Então existiria $\mathrm{T}_{3}:\left[\ell_{1}(\mathbb{N})\right]^{\prime} \rightarrow\left(X^{\prime}\right)^{\prime}$ definida por $\mathrm{T}_{3}\left(\mathrm{x}^{\prime}\right)=$ $=\mathrm{T}_{2}{ }^{\circ} \mathrm{X}^{\prime}$ que $\overrightarrow{\mathrm{e}}$ linear, continua e injetora $\left(\mathrm{T}_{2} \overrightarrow{\mathrm{e}}\right.$ sobre. Veja 
[Y], página 205). Portanto $T_{3}: \ell_{\infty}(\mathbb{N}) \rightarrow X^{\prime \prime}$ e uma função $1 \mathrm{i}-$ near, contínua e injetora de um espaço não separävel num espaço separäve 1; o que è absurdo.

2.b - CLASSES DE ESPAÇOS DE BANACH

Antes de abordarmos os resultados que constam neste parägrafo, convëm introduzir algumas notações que serão frequentemente utilizadas:

Seja E um conjunto, o(E) uma o-älgebra de subconjuntos de $\mathrm{E}$ e u uma medida positiva definida em o(E). L $\mathrm{L}_{\mathrm{p}}(\mathrm{u})$ ou $L_{p}(E, \sigma(E), u), 1 \leq p \leq \infty$, denotará a classe de todas as funções mensuräveis (identificamos as que diferem num conjunto de medida nula); $f: E \rightarrow \mathbb{K}, \quad c o m \mathbb{K}=\mathbb{R}$ ou $(\mathbb{C}$ tais que

$$
\int_{E}|f(t)|^{p} d u<\infty \text { e }|f|=\left(\int_{E}|f(t)|^{p} d u\right)^{\frac{1}{p}}
$$

$L_{\infty}(u)=L_{\infty}(E, \sigma(E), u) \quad \bar{e}$ a classe de todas as funções mensuräveis $f: E \rightarrow \mathbb{K}$ tais que $\|f\|=\sup \underset{t \in E}{\operatorname{ess}}|f(t)|<\infty$.

$C(K)$ designarâ o conjunto das funções contînuas $f: K \longrightarrow \mathbb{C}$, sendo $K$ um conjunto compacto (sempre de Hausdorff).

$C(K, \mathbb{R}) \vec{e}$ o conjunto das funçöes contínuas $f: K \rightarrow \mathbb{R}$, sendo $K$ um conjunto compacto. Em $C(K)$ e $C(K, \mathbb{R})$ consideramos a norma $\|f\|=\sup _{t \in K}|f(t)|$.

DEFINICĀO 2.b.1 - Dado um espaço de Banach $x$, dizemos que $X$ 
$\overrightarrow{\mathrm{e}}$ uniformemente convexo se, para todo $\varepsilon>0$, existe $\delta(\varepsilon)>0$ tal que, para quaisquer $x, y \in x, \operatorname{com}\|x\|=\|y\|=1$ e $\|x-y\| \geq \varepsilon$ temos $\left\|\frac{x+y}{2}\right\| \leq 1-\delta(\varepsilon)$.

As propriedades dos espaços uniformemente convexos que utilizaremos podem ser descritas resumidamente por:

TEOREMA 2.b.2 - 1) Os espaços de Hilbert são uniformemente convexos.

2) Subespaços fechados de espaços un iformemente convexos são espaços uniformemente convexos.

3) Os espaços uniformemente convexos são reflexivos.

4) Para $1<p<\infty, L_{p}(u)$ è um espaço uniformemente convexo.

5) Se $x$ è um espaço uniformemente convexo e $\left\{x_{n}\right\}_{n=1}^{\infty}$ èuma base de Schauder de $X$ então existem constantes A e B positivas e nümeros reais $1<p \leq r<\infty$ tais que:

$$
B\left(\sum_{n=1}^{\infty}\left|a_{n}\right|^{r}\right)^{1 / r} \leq\left\|\sum_{n=1}^{\infty} a_{n} x_{n}\right\| \leq A\left(\sum_{n=1}^{\infty}\left|a_{n}\right|^{p}\right)^{1 / p}
$$

para toda escolha de escalares $\left\{a_{n}\right\}_{n=1}^{\infty}$ para os quais a sêrie $\sum_{n=1}^{\infty} a_{n} x_{n}$ converge.

DEMONSTRAÇÄO - 1) Seja X um espaço de Hilbert. Vale a 1ei do paralelogramo: para todo $x, y \in X$,

$$
\|x+y\|^{2}+\|x-y\|^{2}=2\left(\|x\|^{2}+\|y\|^{2}\right),
$$


e daí segue imediatamente que, se $\|x\|=\|y\|=1$ e $\|x-y\| \geq \varepsilon$ então $\left\|\frac{x+y}{2}\right\| \leq 1-\left[1-\sqrt{1-(\varepsilon / 2)^{2}}\right]$.

2) E decorrência imediata da definição de espaçouniformemente convexo.

3) Veja $[K-G]$, pägina 354 .

4) Veja [C] e [H-S], päginas 232 e 227 .

5) $\operatorname{Vej}$ a $[R C-1]$.

TEOREMA $2 . \mathrm{b} .3-L_{\infty}(\mathrm{u})$ é isométrico a $C(K)$, para algum $\mathrm{K}$ compacto.

DEMONSTRAÇĀO - Veja [D-S], pägina 445, volume I.

Vamos agora introduzir as chamadas funções de Rademacher e a desigualdade de Khintchine, que ser-nos-ão $\bar{u}-$ teis no desenvolvimento do problema 3. Usaremos a função sn: $\mathbb{R} \rightarrow \mathbb{R}$ definida por

$$
\sin (x)= \begin{cases}+1, & x>0 \\ -1, & x<0\end{cases}
$$

$\underline{\text { PROPOSICĀO }} \underline{2 \cdot b \cdot 4}-$ Sejam $r_{n}:[0,1] \longrightarrow \mathbb{R}$ definidas por

$$
r_{n}(t)=\operatorname{sn}\left\{\operatorname{sen}\left(2^{n} \pi t\right)\right\}, \text { para todo } t \in[0,1],
$$

$\mathrm{n}=0,1,2,3, \ldots$ (denominadas funções de Rademacher). Para todo $\mathrm{p}$, $1 \leq \mathrm{p}<\infty$, existem nümeros $\mathrm{A}(\mathrm{p}), \mathrm{B}(\mathrm{p})>0$ tais que

$$
A(p)\left(\sum_{n=1}^{m}\left|a_{n}\right|^{2}\right)^{1 / 2} \leq\left(\int_{0}^{1}\left|\sum_{n=1}^{m} a_{n=n} r_{n}(t)\right|^{p} d t\right)^{1 / p} \leq B(p)\left(\sum_{n=1}^{m}\left|a_{n}\right|^{2}\right)^{1 / 2},
$$


para toda escolha de escalares $\left\{a_{n}\right\}_{n=1}^{m}$, e para todo m natural.

DEMONSTRAÇĀO - Veja $[Z]$.

Para as definições que seguem consulte se necessärio, [L-E], päginas 9, 17, 21 e 131 .

$\underline{\text { DEFINIÇĀO }} \underline{2 . b .5}$ - Seja X um reticulado de Banach (real ou complexo). Dizemos que a norma de $X$ é $p$-aditiva $(1 \leq p<\infty)$, se, para todo $x, y \in X$ com $x \wedge y=0$ vale que $\|x+y\|^{p}=\|x\|^{p}+\|y\|^{p}$. Um reticulado de Banach em que a norma è p-aditiva $(1 \leq \mathrm{p}<\infty)$ è chamado um espaço L $\mathrm{L}$-abstrato.

$\underline{\text { TEOREMA }} \underline{2 . \mathrm{b} .6}$ - Seja X um espaço $\mathrm{L}_{\mathrm{p}}$-abstrato.

1) $\mathrm{X}$ é isomëtrico a $\left[\ell_{p}(\Gamma, \mathbb{L}) \oplus\left(\oplus \sum_{\alpha \in A} L_{p}\left([0,1]^{m}, \mathbb{C}\right)\right)_{p}\right]_{p}$, para algum conjunto de indices $\Gamma$ e algum conjunto de cardinais $\left\{m_{\alpha}\right\}_{\alpha \in A}$, com $m_{\alpha} \geq x_{0}$, para todo $\alpha \in A$.

2) Se $m \geq x_{0}$ então $L_{p}\left([0,1]^{m}, \mathbb{C}\right)$ contẻm um subespaço isomorfo a $\ell_{2}(\Gamma, \mathbb{C})$, onde card $\Gamma=\mathrm{m}, 1 \leq \mathrm{p}<\infty$.

DEMONSTRAÇĀO - 1) Veja [L-E], corolärio pägina 136.

2) Veja [L-E], pägina 128 , teorema 12 .

TEOREMA $\underline{2 \cdot b \cdot 7}$ - 1) Seja $x$ um espaço de Banach sobre o corpo dos reais que contêm um subespaço isomorfo a $\ell_{1}(\mathbb{N}, \mathbb{R})$. Então $X^{\prime}$ tem um subespaço isomorfo a $L_{1}\left(\{0,1\}^{\mathbb{N}}\right)$.

2) Se u é uma medida positiva não atômica, então existe 
um conjunto enumerävel $\left\{\mathrm{m}_{B} \mid B<\alpha\right\}$ de cardinais distintos; $\left(\mathrm{m}_{B}{ }^{2}\right.$ $\geq x_{0}$ ) tal que $L_{p}(u), 1 \leq p<\infty \vec{e}$ isomorfo a

$$
\text { [. } \left.\oplus \sum_{B<\alpha} L_{p}\left([0,1]^{m_{B}}, \mathbb{K}\right)\right]_{p} \text {. }
$$

3) Se $K \vec{e}$ um espaço compacto (Hausdorff) săo equivalentes as propriedades:

a) $K$ è disperso.

b) $[C(K, \mathbb{R})]^{\prime}$ ë isomorfo a $\ell_{1}(\Gamma, \mathbb{R})$ para algum $\Gamma$.

c) $[\mathrm{C}(\mathrm{K}, \mathbb{R})]^{\prime}$ não contëm subespaço isomorfo a

$$
L_{1}\left([0,1]^{\mathbb{N}}, \mathbb{R}\right) .
$$

DEMONSTRAC̄ĀO - 1) Veja [R-H], pägina 316, proposição 2.2 .

2) Veja $[\mathrm{L}-\mathrm{E}]$, pägina 127 , teorema 9.

3) Veja [P-S], pägina 214, itens 1, 12 e 13. CQD

No capitulo 3 classificamos a importante classe de espaços de Banach que passamos a definir.

DEFINICAAO $2 . b \cdot 8$ - Seja $1 \leq p<\infty$ e seja $\omega=\left\{\omega_{n}\right\}_{n=1}^{\infty}$ uma sequência não crescente de nümeros positivos tais que

$$
\omega_{1}=1, \lim _{n \rightarrow \infty} \omega_{n}=0 \text { e } \sum_{n=1}^{\infty} \omega_{n}=\infty \text {. }
$$

0 espaço de Banach de todas as sequências de escalares $x=$ $=\left(a_{1}, a_{2}, a_{3}, \ldots\right)$ para as quais

$$
\|x\|=\sup _{\pi}\left(\sum_{n=1}^{\infty}\left|a_{\pi(n)}\right|^{p} \omega_{n}\right)^{1 / p}<\infty,
$$

(onde $\pi$ percorre todas as permutações dos inteiros) serä de- 
notado por $\mathrm{d}(\omega, \mathrm{p})$ e $\vec{e}$ chamado espaço de sequências de Lorentz (veja $[L])$.

EXEMPLO 2.b.9 - Seja $\mathrm{T}_{0}$ o conjunto das sequências quase nu1as. Denotaremos por $\left\{e_{n}\right\}_{n=1}^{\infty}$ os vetores

$$
\mathrm{e}_{\mathrm{n}}=(0,0,0, \ldots 01,000 \ldots)
$$

$e$, se $x=\sum_{n=1}^{\infty} a_{n} e_{n} \in T_{0}$, tomemos $\|x\|_{0}=\max _{n \in \mathbb{N}}\left|a_{n}\right| e$

$$
\|x\|_{m+1}=\max \left\{\|x\|_{m}, \frac{1}{2} \max \sum_{j=1}^{K}\left\|_{n=p_{j}+1}^{P_{j}+1} a_{n} e_{n}\right\|_{m}\right\}
$$

onde o mäximo interior à chave è tomado sobre todas as escolhas de $\mathrm{K} \leq \mathrm{p}_{1}<\mathrm{p}_{2}<\ldots<\mathrm{p}_{\mathrm{K}+1}, \mathrm{~K}=1,2,3, \ldots$.

E imediato que, se tomarmos $\|x\|=\lim _{m \rightarrow \infty}\|x\|_{m}$ (que existe, pois a sucessão $\|x\|_{m}$ é crescente e limitada superiormente), obtemos uma norma em $\mathrm{T}_{0}$. Denotaremos por $\mathrm{T}$ o completado de $\mathrm{T}_{0}$.

São conhecidos os resultados seguintes:

1) $\|x\|=\max \left\{\max _{n \in \mathbb{N}}\left|a_{n}\right|, \frac{1}{2} \sup \left(\sum_{j=1}^{K}\left\|\sum_{n=p_{j}+1}^{p_{j+1}} a_{n}{ }_{n}\right\|\right.\right.$, $\left.\left.\mathrm{K} \leq \mathrm{p}_{1}<\mathrm{p}_{2}<\ldots<\mathrm{p}_{\mathrm{K}+1}, \mathrm{~K}=1,2,3 \ldots\right)\right\}$ para todo $\mathrm{x} \in \mathrm{T}$.

2) $\left\{e_{n}\right\}_{n=1}^{\infty}$ è uma base incondicional de T. (Veja definição 2.a.1) .

3) T não contém subespaço isomorfo a $c_{0}(\mathbb{N})$ ou $\ell_{1}(\mathbb{N})$.

4) T é reflexivo - E decorrência imediata do 2) e 3). (Veja teorema 2.a.12). 
5) T não contẻm nenhum subespaço de dimensão infinita uniformemente convexo.

DEMONSTRACĀO - Veja $[\mathrm{F}-\mathrm{J}]$.

DEFINIÇĀO 2.b.10 - Um espaço de Banach $X$ é denominado um espaço de Schurr, se para toda sequência $\left\{x_{n}\right\}_{n=1}^{\infty}$ a propriedade $x_{n} \stackrel{\text { fracamente }}{\rightarrow} 0$ implica que $\left\|x_{n}\right\| \stackrel{n \rightarrow \infty}{\longrightarrow} 0$.

PROPOSIÇĀO 2.b.11 - 1) Seja $\Gamma$ um conjunto não vazio,

$$
\mathbb{K}=\mathbb{R} \text { ou } \mathbb{C} ; \ell_{1}(\Gamma, \mathbb{K})=\left\{f: \Gamma \rightarrow \mathbb{K}\left|\|f\|=\sum_{x \in \Gamma}\right| f(x) \mid<\infty\right\}, \ell_{1}(\Gamma, \mathbb{K})
$$

$\vec{e}$ um espaço de Schurr.

2) Todo espaço de Schurr então, ê um espaço de Banach, fracamente sequencialmente completo.

$\underline{\text { DEMONSTRAÇĀO }}$ - 1) Veja [L-E], pägina 174 , 1ema 4.

2) Seja $\left\{x_{n}\right\}_{n=1}^{\infty}$ uma sequência de Cauchy fraca,então dadas quaisquer subsequências $\left\{x_{n_{r}}\right\}_{r=1}^{\infty}\left\{x_{m_{r}}\right\}_{r=1}^{\infty}$ de $\left\{x_{n}\right\}_{n=1}^{\infty}$ temos que $y_{r}=x_{n_{r}}-x_{m_{r}} ; y_{r} \frac{\text { fracamente }}{r \rightarrow \infty} 0$. Como $x$ é um espaço de Schurr $\left\|y_{\mathrm{r}}\right\| \underset{\mathrm{r} \rightarrow \infty}{\longrightarrow} 0$ logo a sequência $\left\{\mathrm{x}_{\mathrm{n}}\right\}_{\mathrm{n}=1}^{\infty}$ tom a propriedade: Dadas duas subsequências quaisquer

$$
\left\{x_{n_{r}}\right\}_{r=1}^{\infty},\left\{x_{m_{r}}\right\}_{r=1}^{\infty},\left\|x_{n_{r}}-x_{m_{r}}\right\| \underset{n \rightarrow \infty}{\longrightarrow} 0
$$

e então $\left\{x_{n}\right\}_{n=1}^{\infty}$ é de Cauchy na norma do espaço, logo

$$
\left\|x_{n}-x_{0}\right\| \underset{n \rightarrow \infty}{\longrightarrow} 0 \text { e portanto } x_{n} \frac{\text { fracamente }}{n \rightarrow \infty} x_{0} \text {. }
$$




\section{CAPITUL.0 3}

\section{PROBLEMAS}

3.a - PROBLEMAS DOIS, CINCO E APLICAÇÖES

DEFINTCĀO 3.a.1 - Sejam X e Y espaços de Banach e

$$
\operatorname{fESV}([a, b], L(X, Y)) \text {. }
$$

Para $[c, d] c[a, b]$ definimos:

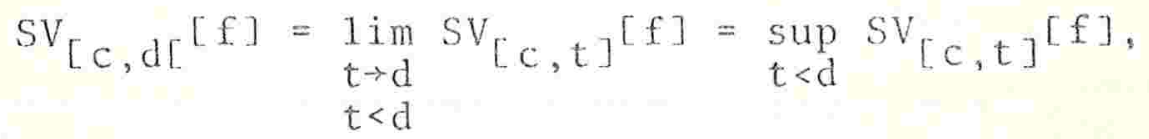

e analogamente.

$$
\mathrm{SV}_{] c, d]}[f]=\lim _{\substack{t \rightarrow c \\ t>c}} S V_{[t, d]^{[f]}=\sup _{t>c} S V_{[t, d]}[f] .}
$$

Quando $X=\mathbb{K} \quad(\mathbb{K}=\mathbb{R}$ ou $\mathbb{C})$ usaremos as notaçöes

$$
\begin{aligned}
& { }_{\left[c, d\left[{ }^{[f]}, W^{W}\right] c, d\right]}[f] \text { em vez de } S V_{\left[c, d[f], S V_{] c}, d\right]}[f] \\
& \text { o teorema } 3, a, 2 \text { e o corolärio } 3 . a .6 \text { que seguem re- }
\end{aligned}
$$
solvem completamente os problemas $[\mathrm{P}-5]$ e $[\mathrm{P}-2]$ propostos no capitulo 1 . 
TEOREMA $3 . a .2$ - Sejam X e $Y$ espaços de Banach, com $X \neq\{0\}$. São equivalentes as seguintes propriedades:

1) Y não contêm um subespaço isomorfo a $\mathcal{C}_{0}(\mathbb{N})$.

2) Para toda $f \in S V([a, b], L(X, Y))$, temos:

a) $\lim _{\varepsilon \rightarrow 0} S V_{[t-\varepsilon, t[}[f]=0$, para qualquer $\left.\left.t \in\right] a, b\right]$; $\varepsilon>0$

b) $\left.\lim _{\varepsilon \rightarrow 0} S V_{t}, t+\varepsilon\right]^{[f]}=0$, para qualquer $t \in[a, b[$. $\varepsilon>0$

3) $\operatorname{SV}([a, b], L(X, Y)) \subset G([a, b], L(X, Y))$.

4) $\operatorname{SV}([a, b], L(X, Y)) \subset D([a, b], L(X, Y))$.

DEMONSTRAÇĀO -1$) \Longrightarrow$ 2) Se não, suponhamos que exista $t_{0} \in[a, b]$

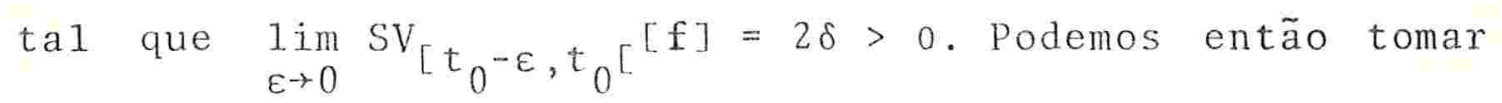
$\varepsilon>0$

$\left\{q_{n}\right\}_{n=1}^{\infty} c[a, b]$, estritamente crescente, com $\lim _{n \rightarrow \infty} q_{n}=t_{0}$ e tal que $\mathrm{SV}_{\left[\mathrm{q}_{\mathrm{n}}, \mathrm{q}_{\mathrm{n}+1}\right]}[f]>\delta$, para todo $n$ natura 1 . Como

$$
\operatorname{fESV}([\mathrm{a}, \mathrm{b}], \mathrm{L}(\mathrm{X}, \mathrm{Y}))
$$

para todo n natural, existe

$$
q_{n}=t_{0}^{n}<t_{1}^{n}, \ldots,<t_{j(n)}^{n}=q_{n+1} \text { e } x_{i} \epsilon X,\left\|x_{i}\right\| \leq 1,1 \leq i \leq j(n),
$$

tais que, se

$$
h_{n}=\sum_{i=1}^{j(n)}\left[f\left(t_{i}^{n}\right)-f\left(t_{i-1}^{n}\right)\right] x_{i}
$$

então 


$$
\left\|h_{n}\right\|=\left\|\sum_{i=1}^{j(n)}\left[f\left(t_{i}^{n}\right)-f\left(t_{i-1}^{n}\right)\right] x_{i}\right\| \geq \frac{\delta}{2} \text {. }
$$

Por outro 1 ado, como $\operatorname{fESV}([a, b], L(X, Y))$ então

$$
\sup _{\left|\lambda_{i}\right| \leq 1}\left\|\sum_{n=1}^{K} \lambda_{n} h_{n}\right\| \leq \operatorname{sV}[f]
$$

KEIN

e aplicando o corolärio 2.a.14, virả que Y contém subespaço isomorfo a $C_{0}(\mathbb{N})$, o que contraria a hipötese.

2) $\Longrightarrow 3$ ) Considere $t_{0} E[a, b]$ e $\left\{t_{n}\right\}_{n=1}^{\infty}$ uma sequência estritamente crescente (ou decrescente) convergindo a $\mathrm{t}_{0}$. Como $\left.\left\|f\left(t_{n}\right)-f\left(t_{m}\right)\right\| \leq S V_{\left[t_{n}\right.}, t_{m}\right][f], \vec{e}$ imediato, aplicando 2$)$, que $\left\{f\left(t_{n}\right)\right\}_{n=1}^{\infty} \vec{e}$ uma sequência de Cauchy; portanto, existe

$$
\lim _{t \rightarrow t_{0}^{-}} f(t)
$$

$3) \Longrightarrow$ 4) Imediato pe1o corolärio 1.c.3, item 3.

$4) \Longrightarrow$ 1) Seja $H$ um subespaço de $Y$ isomorfo a $C_{0}(\mathbb{N})$, e $\mathrm{J}: \mathrm{C}_{0}(\mathbb{N}) \rightarrow \mathrm{H}$ um isomorfismo. Em 1.e.2, mostramos que existe $f:[a, b] \rightarrow c_{0}(\mathbb{N})$ tal que $f \in B W\left([a, b], c_{0}(\mathbb{N})\right)$ e $f q D\left([a, b], C_{0}(\mathbb{N})\right)$. Portanto, para $h=J$ of, temos $h \in B W([a, b], Y)$ e $h \notin D([a, b], Y)$. Seja $x_{0}^{\prime} \in X^{\prime} \operatorname{com}\left\|x_{0}^{\prime}\right\|=1$, e $\hat{\mathrm{f}}:[\mathrm{a}, \mathrm{b}] \longrightarrow L(X, Y)$, definida por $\hat{f}(t)(x)=x_{0}^{\prime}(x) h(t)$, para todo $t \in[a, b]$ e todo $x \in X$. E imediato que $\hat{f} \in \operatorname{SV}([a, b], L(X, Y))$ e, a1ém disto, $\hat{f} \notin D([a, b], L(X, Y))$, o que contraria a hipótese.

CQD

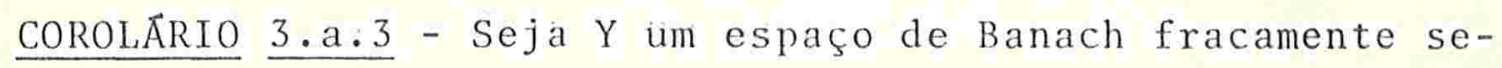


quencialmente completo (em particular, reflexivo). São verdadeiras as afirmações equivalentes 2,3 e 4 do teorema $3 . a .2$. DEMONSTRAÇĀO - Pela proposição $2 . a .22$, sabemos que, se Y ë fracamente sequencialmente completo então Y não contém nenhum subespaço isomorfo a $C_{0}(\mathbb{N})$; do teorema $3 . a .2$ segue a conclusão.

EXEMPLO $\underline{3 . a .4}$ - Considere J o espaço de James, formado por todas as sequências $x=\left(a_{1}, a_{2}, \ldots, a_{n} \ldots\right)$ de escalares para as quais vale:

a) $\|x\|=\sup \frac{1}{\sqrt{2}} \sqrt{\left(a_{p_{1}}-a_{p_{2}}\right)^{2}+\left(a_{p_{2}}-a_{p_{3}}\right)^{2}+\ldots+\left(a_{p_{m-1}}-a_{p_{m}}\right)^{2}+\left(a_{p_{m}}-a_{p_{1}}\right)^{2}}<\infty$, o supremo sendo tomado sobre todas as escolhas de m€IN e $\mathrm{p}_{1}<\mathrm{p}_{2}<\ldots<\mathrm{p}_{\mathrm{m}}$

b) $\lim _{n \rightarrow \infty} a_{n}=0$.

Seja $e_{i}=(0,0, \ldots, 0, \stackrel{i}{1}, 0,0, \ldots)$. Temos:

1) $J^{\prime \prime}=\left\{\left(a_{1}, a_{2}, \ldots, a_{n} \ldots\right) \mid \sup _{n \in \mathbb{N}}\left\|\sum_{i=1}^{n} a_{i} e_{i}\right\|<\infty\right\}$

2) A imagem de $J$ em J" pela aplicação canônica tem codimensão 1 em J", e portanto, J não ê reflexivo.

3) J" é separäve1, donde decorre que $J$ não contêm $C_{0}(\mathbb{N})$ ou $\ell_{1}(\mathbb{N}) \cdot($ Veja proposição $2 \cdot a \cdot 26)$.

4) J e J" são isométricos (não pela aplicação canônica) .

DEMONSTRAÇĀO - Veja [R-C-2], [R-C-3]. 
PROPOSICÃO 3.a.5 - Seja J o espaço de James (exemplo 3.a.4). Temos:

1) J não é fracamente sequencialmente completo.

2) São verdadeiras as propriedades equivalentes 2,3 e 4 do teorena $3 . \mathrm{a} .2$ onde $Y=J$.

DEMONSTRACĀO - 1) Por 2 do exemplo 3.a.4, sabemos que J não ë reflexivo. Por outro lado, 3, do mesmo exemplo, garante que J não contêm $\ell_{1}(\mathbb{N})$, e aplicando o corolärio $2 . a .25$, demonstramos 1).

2) f̂ decorrência imediata de 3 do exemplo $3 . a .4$ e do teorema $3 \cdot a \cdot 2$. CQQD

COROLÄrIO 3.a.6 - Seja Y um espaço de Banach. São equivalentes as propriedades:

1) $Y$ não contëm subespaço isomorfo a $C_{0}(\mathbb{N})$.

2) Para toda $f \in B(\omega([a, b], X)$, temos:

a) $\lim _{\varepsilon \rightarrow 0} W_{[t-\varepsilon, t[}[f]=0$, para qualquer $\left.\left.t \in\right] a, b\right]$; $\varepsilon>0$

b) $\left.\left.\lim _{\varepsilon \rightarrow 0} w_{t}\right] t, t+\varepsilon\right]^{[f]}=0$, para qua]quer $t \in[a, b[$. $\varepsilon>0$

3) $B W([a, b], Y) \subset C([, a, b], Y)$.

4) $B W([a, b], Y) \subset D([a, b], Y)$.

DEMONSTRACÄO - Basta aplicar o teorema $3 . a .2$ para o caso $X=$ $=\mathbb{I}, \mathbb{K}=\mathbb{R}$ ou $\mathbb{d} \cdot($ Veja definição $1 . \mathrm{d} .7$, item c). CQQD 
0 corolärio abaixo contém os teoremas $4.12,4.13$ e 4.14 de $[H-1]$, páginas 49,50 e 51 .

COROLARIO 3.a.7 - Seja Y um espaço de Banach fracamente sequencialmente completo (em particular, reflexivo).Então são verdadeiras as propriedades equivalentes 2,3 e 4 do coro1 ärio $3 \cdot a \cdot 6$.

DEMONSTRAÇĀO - Se Y $\overrightarrow{\mathrm{e}}$ um espaço de Banach fracamente sequencialmente completo, sabemos, pela proposição $2 . a .22$, que Y não contém subespaço isomorfo a $C_{0}(\mathbb{N})$; aplicando o corolärio $3 . a .6$, obtemos imediatamente o resultado. CQD

A proposição que se segue mostra que o corolärio $3 . a .6$ é mais geral que o coroläxio $3 . a .7$, e portanto, mais geral que os teoremas $4.12,4.13$ e 4.14 de [H-1]

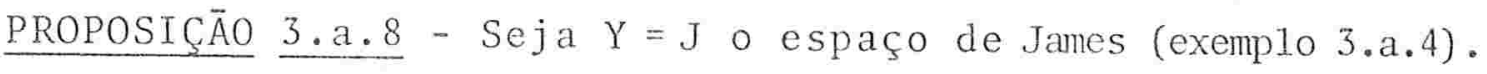
Temos:

1) J não è fracamente sequencialmente completo.

2) São verdadeiras as propriedades equivalentes 2,3 e 4 do corolärio $3 . \mathrm{a} \cdot 6$, onde $Y=J$.

DEMONSTRAÇĀO - 1) E decorrência imediata da proposição 3.a.5.

2) E decorrência imediata de 3 do exemplo $3 . a .4$ e do corolärio $3 \cdot a \cdot 6$. CQD

$\underline{3 . a .9}$ APLICAÇOEES DOS PROBLEMAS DOIS E CINCO - Queremos agora mencionar outras questões em aberto resolvidas pelos pro- 
blemas $[\mathrm{P}-2]$ e $[\mathrm{P}-5]$.

$[P-6]$ Se $\operatorname{SV}([a, b], L(X, Y)) \subset G([a, b], L(X, Y))$ temos necessariamente que Y $\vec{e}$ fracamente sequencialmente completo? (Veja [H-5], pägina 13, problema 5). A resposta é negativa, como decorrência imediata da proposição 3.a.5.

[P-7] Uma outra questão em aberto, cuja resolução foi encaminhada pelo problema $[\mathrm{P}-2]$ e $[\mathrm{P}-5]$ é:

Seja $\operatorname{fESV}([a, b], L(X, Y))$ e $t_{0} \in\left[a, b\left[\right.\right.$ ta 1 que existe $\lim _{t \downarrow t_{0}} f(t)=$ $=f\left(t_{0}+\right) \cdot$ Podemos concluir que

$$
\left.\lim _{\substack{\varepsilon \rightarrow 0 \\ \varepsilon>0}} S V_{0}, t_{0}+\varepsilon\right][f]=0 ?
$$

(Veja $[\mathrm{H}-2]$, pägina 185 , problema 7.4$)$.

O problema [P-5] mostra que, se existe uma tal função, o contra domínio deverá conter $C_{0}(\mathbb{N})$ (a menos de isomorfismo). De fato, seja $f:[0,1] c \mathbb{R} \rightarrow C_{0}(\mathbb{N})$, definida por

$$
f(x)= \begin{cases}0, & x \notin\left\{\frac{1}{n}\right\}_{n=1}^{\infty} \\ f_{n}, & \text { se } x=\frac{1}{n}\end{cases}
$$

onde $f_{1}=(1,0,0,0, \ldots), f_{2}=\left(0, \frac{1}{2}, 0,0, \ldots\right), f_{3}=\left(0, \frac{1}{2}, 00, \ldots\right)$, $f_{4}=\left(0,0, \frac{1}{3}, 0, \ldots\right), f_{5}=\left(0,0, \frac{1}{3}, 0, \ldots\right), f_{6}=\left(0,0, \frac{1}{3}, 0, \ldots\right), \ldots$ Dada uma sequência $\delta=\left\{s_{1}, t_{1}, s_{2}, t_{2}, \ldots, s_{n}, t_{n}\right\}$, com $0 \leq s_{1}$ $<t_{1} \leq \ldots \leq s_{n}<t_{n} \leq 1$, vem imediatamente que 


$$
\left\|\sum_{i=1}^{n}\left[f\left(t_{i}\right)-f\left(s_{i}\right)\right]\right\| \leq 1
$$

e portanto, $f \in B W\left([0,1], C_{0}(\mathbb{N})\right)$. Por outro 1 ado, dado $\left.] 0, \varepsilon\right], \vec{e}$ imediato que

$$
W] 0, \varepsilon]^{[f]=1, \text { e portanto, }} \lim _{\substack{\varepsilon \rightarrow 0 \\ \varepsilon>0}} w_{0, \varepsilon]}=1 .
$$

Entretanto, um cálculo simples mostra que $\lim _{t \rightarrow 0} f(t)=0=f(0)$.

$$
\text { Seja } \hat{\mathrm{f}}:[0,1] \longrightarrow L(X, Y) \text {, definida por }
$$

$$
\hat{f}(t) \cdot x=x_{0}^{\prime}(x) \cdot f(t), \quad c o m x_{0}^{\prime} \in X^{\prime},\left\|x_{0}^{\prime}\right\|=1 \text {. }
$$

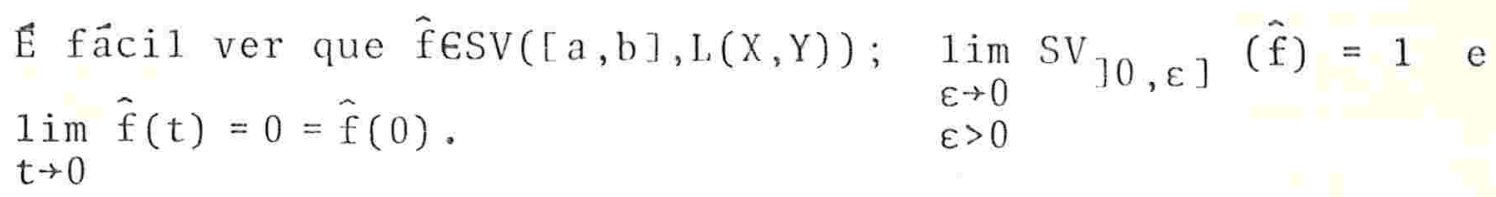

[P-8] E claro que podemos obter $f_{1}:[0,1] \subseteq \mathbb{R} \rightarrow C_{0}(\mathbb{N})$ contínua, com as mesmas propriedades da função $f$ definida em [P-7]. (Basta tomar $f$ nula nos pontos médios dos intervalos $\left[\frac{1}{n+1}, \frac{1}{n}\right]$ e linearizar entre 2 pontos consecutivos de definição).

Então se $X \neq\{0\}$, a função $\hat{f}_{1}:[0,1] \subseteq \mathbb{R} \rightarrow L\left(X, C_{0}(\mathbb{N})\right)$ è dada por $\hat{f}_{1}(t)(x)=x_{0}^{\prime}(x) \cdot f_{1}(t)$, onde $x_{0}^{\prime} \in X^{\prime},\left\|x_{0}^{\prime}\right\|=1$ têm as seguintes propriedades:
a) $\hat{f}_{1} \operatorname{eCSV}([0,1], L(X, Y))$.
b) $\operatorname{SV}_{[0, \delta]^{\left[\hat{f}_{1}\right]}}=1,0<\delta<1$.
c) $\mathrm{SV}_{[0,1]}\left[\hat{\mathrm{f}}_{1}\right]=1$. 
o que resolve outra questão em aberto: (veja [H-2], pägina 186, problema 7.5 ).

Dar um exemplo de $\operatorname{E\in CSV}([a, b], L(X))$ e deD tal que

$$
\mathrm{SV}_{\mathrm{d}}[f]-\left\|f\left(t_{1}\right)-f(\mathrm{a})\right\|>\operatorname{SV}[f]-\mathrm{SV}_{\left[\mathrm{a}, \mathrm{t}_{1}\right]}[\mathrm{f}]
$$

De fato, tomando-se $f=\hat{f}_{1},[a, b]=[0,1], a=0$ e $t_{1} \neq 0, t a 1$ que $\hat{f}_{1}\left(t_{1}\right)=0$, virá que $S_{d}\left[\hat{f}_{1}\right]>0$, o que ë obviamenteverdadeiro para toda partição d que contenha o ponto $t_{1}$.

[E-1] Em [H-2], pägina 185, após o problema 7.4, Hönig afirma que, se $f \in B \omega([a, b], x)$ e existe $f\left(t_{0}^{+}\right)$então

$$
\left.\left.\lim _{\substack{\varepsilon \rightarrow 0 \\ \varepsilon>0}} B W\right] t_{0}, t_{0}+\varepsilon\right][f]=0 .
$$

A função $f:[0,1] \subseteq \mathbb{R} \longrightarrow c_{0}(\mathbb{N})$,

$$
f(x)=\left\{\begin{array}{l}
0, \quad x \notin\left\{\frac{1}{n}\right\}_{n=1}^{\infty} \\
f_{n}, \text { se } x=\frac{1}{n}
\end{array}\right. \text { (vide solução do problema [P-7]). }
$$

mostra que a afirmação é incorreta.

[E-2] Em [H-1], pägina 48, Hönig demonstra que, se $f \in B W([a, b], X)$ então, para todo $t \in\left[a, b\left[, f\left(t^{+}\right) \in X^{\prime \prime}\right.\right.$ existe no sentido $X_{\sigma}^{\prime \prime}\left(X^{\prime \prime}, X^{\prime}\right)$ - A seguir, demonstra o teorema:

Seja X um espaço de Banach e $f \in B(U([a, b], X)$. Se $t \in\left[a, b\left[\right.\right.$ e $f\left(t^{+}\right) \in X$ então 1 im $\left\|f(t+\varepsilon)-f\left(t^{+}\right)\right\|=0$. $\varepsilon \rightarrow 0$ 
Demos o seguinte contra exemplo ao resultado acima: seja

$$
f:[0,1] \subseteq \mathbb{R} \rightarrow C_{0}(\mathbb{N}), f(x)=\left\{\begin{array}{l}
(0,0 \ldots 0,1,0 \ldots) \text { se } x=\frac{1}{n} \\
0, \quad x \in\left\{\frac{1}{n}\right\}_{n=1}^{\infty}
\end{array}\right.
$$

E imediato que $\mathrm{f} \in \mathrm{BW}\left([0,1], \mathrm{C}_{0}(\mathbb{N})\right)$ e que $\mathrm{f}\left(0^{+}\right)=0 \in\left[\mathrm{C}_{0}(\mathbb{N})\right]^{\prime \prime}=\ell_{\infty}(\mathbb{N})$ (na topologia $\sigma\left(X^{\prime \prime}, X^{\prime}\right)$ ). Tambëm temos $f\left(0^{+}\right)=0 \in C_{0}(N)$, mas não existe $\lim _{\varepsilon \rightarrow 0}\left\|f(\varepsilon)-f\left(0^{+}\right)\right\|$.

$$
\varepsilon>0
$$

Para terminar, queremos observar que, se Ynão contem $C_{0}(\mathbb{N})$ e $\operatorname{fESV}([a, b], L(X, Y))$ então, pelo teorema $3 . a .2$, temos:

$$
\left.\lim _{\substack{\varepsilon \rightarrow 0 \\ \varepsilon>0}} S V_{0}, t_{0}+\varepsilon\right]^{[f]=0} \text { e } f \in G([a, b], L(X, Y))
$$

Segue, como consequência deste resultado, uma resposta parcial aos problemas $7.3,7.7$ de $[H-2]$ : Se $x \vec{e}$ um espaço de Banach que não contém subespaço isomorfo a $C_{0}(\mathbb{N})$ então o sistema

$$
\left\{\begin{array}{l}
\left.y(t)-y(s)+\int_{S}^{t} d A(\sigma) y(\sigma)=f(t)-f(s), \text { para todo } t, s \in\right] a, b[ \\
F(y)=c
\end{array}\right.
$$

onde

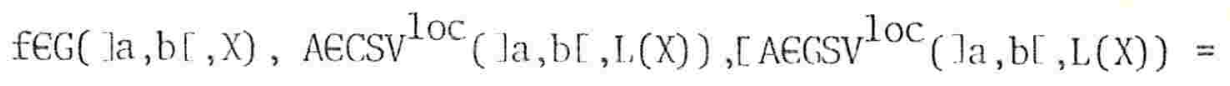

$$
\begin{aligned}
& =\mathrm{SV}^{10 \mathrm{c}}(\mathrm{a}, \mathrm{b}[, \mathrm{L}(\mathrm{X}))], \mathrm{FEL}(\mathrm{G}(] \mathrm{a}, \mathrm{b}[, \mathrm{X}), \mathrm{Y}) \text { e } \mathrm{cEY} F=\mathrm{F}[\mathrm{G}(] \mathrm{a}, \mathrm{b}[, \mathrm{X})] \text {, }
\end{aligned}
$$


tem uma ünica solução (pois existe o resolvente)

$$
y \in G(] a, b[, x) \text {. }
$$

Tal resultado era conhecido para a classe dos espaços X fracamente sequencialmente completos. Agora a classe foi ampliada como mostra, outra vez, o espaço J de James. Resta ainda o problema de saber se a equação (I) tem reso1vente quando $X$ contêm $C_{0}(\mathbb{N})$. (Por exemplo, nos casos importantes de $x=C([a, b])$ ou $G([a, b]))$.

Vamos agora mencionar rapidamente o problema damensurabilidade das funções dos espaços

$$
B W([a, b], Y) \text { e } R([a, b], Y) \text {. }
$$

$\underline{\text { DEFINICĀO }} \underline{3 . a .10}$ - Seja Y um espaço de Banach e $\mathrm{f}:[\mathrm{a}, \mathrm{b}] \rightarrow \mathrm{Y}$.

a) $f:[a, b] \rightarrow Y$ serä chamada uma função simples se existem conjuntos mensuräveis dois a dois disjuntos, $B_{1}, B_{2}, \ldots$, $B_{m}$, de $[a, b]$, tais que $f(t)=\sum_{i=1}^{m} x_{B}(t) \cdot x_{j}$, onde $x_{j} \in Y$ e $x_{B_{j}}$ é a função característica de $B_{j}$.

b) $\mathrm{f}:[\mathrm{a}, \mathrm{b}] \longrightarrow \mathrm{Y}$ serä dita fortemente mensuräuel se existe uma sequência $f_{n}$ de funçöes simples tais que $f_{n}(t) \longrightarrow f(t)$, para quase todo $t \in[a, b]$. Denotaremos por $M([a, b], Y)$ o espaço vetorial das funções mensurảveis.

c) $\mathrm{f}:[\mathrm{a}, \mathrm{b}] \longrightarrow \mathrm{Y}$ serä dita fracamente mensurāuel se $x^{\prime} \circ f:[a, b] \longrightarrow \mathbb{K} \ddot{e}$ mensuräve1, para toda $x^{\prime} \in X^{\prime}$.

TEOREMA 3.a.11 (Pettis) - Seja Y espaço de Banach separävel 
$f:[a, b] \longrightarrow Y$. São equivalentes as propriedades:

a) $f$ é fortemente mensuráve1.

b) $f \bar{e}$ fracamente mensurävel.

DEMONSTRAÇĀO - Veja $[Y]$.

COROLARIO 3.a.12 (Pettis) - Seja Y um espaço de Banach separäve1. Temos:

1) $R([a, b], Y) \subset M([a, b], Y)$.

2) $B W([a, b], Y) \subset M([a, b], Y)$.

DEMONSTRAÇĀO - 1) Basta lembrar que, se $f \in R([a, b], Y)$ então $x^{\prime} \circ f \in R([a, b], \mathbb{K})$, para toda $x^{\prime} \in X^{\prime}$, por 2 da proposição 1.a.7, e aplicando 3.a.11, temos que $f \in M([a, b], Y)$.

2) E imediato, pois $B(W([a, b], Y) \subset R([a, b], Y)$ (por 6 do teorema $1 . d .4)$.

CQD

Os exemplos abaixo mostram que, para espaços de Banach não separáveis, o corolärio 3.a.12 não é verdadeiro.

EXEMPLOS $3 . a .13$ - 1) Seja H um espaço de llilbert com uma base Hilbertiana $\left\{\ell_{t}\right\}_{0 \leq t \leq 1}$. Definimos $f:[0,1] \longrightarrow H$ por $f(t)=e_{t} \cdot$ E imediato que:

a) $f \in R([0,1], H)$ e $\int_{0}^{1} f(t) d t=0$;

b) $f \mathscr{f}([0,1], \mathrm{H})$.

DEMONSTRACĀOO - a) $\left\|\sum_{i=1}^{|\mathrm{d}|} f\left(\varepsilon_{i}\right) \Delta t_{i}\right\| \leq \sqrt[2]{\sum_{i=1}^{|\mathrm{d}|}\left(\Delta t_{i}\right)^{2}} \leq \sqrt{\Delta \mathrm{d}}$. 
Portanto,

$$
\lim _{\Delta d \rightarrow 0} \sum_{i=1}^{|d|} f\left(\varepsilon_{i}\right) \Delta t t_{i}=\int_{0}^{1} f(t) d t=0 .
$$

b) Dado $\varepsilon>0$, é imediato que não existe um compacto $K_{\varepsilon} c[0,1]$ tal que $u\left(\mathrm{cK}_{\varepsilon}\right) \leq \varepsilon, \operatorname{com} f / K_{\varepsilon}$ contínua; logo, $f$ não ê mensurảvel.

2) Sejam $I=[0,1], Y=C_{0}(I)$ e $\ell_{t}:[0,1] \rightarrow \mathbb{K}$, definida por

$$
\ell_{t}(x)= \begin{cases}0, & t \neq x \\ 1, & t=x\end{cases}
$$

A função $f:[0,1] \longrightarrow C_{0}(I)$, dada por $f(t)=\ell_{t}$, tem as seguintes propriedades:

a) $f \in B W\left([0,1], C_{0}(I)\right)$ (e portanto, $\left.f \in R\left([0,1], C_{0}(I)\right)\right)$;

b) $\mathrm{f} \notin M\left([0,1], \mathrm{C}_{0}(\mathrm{I})\right)$.

DEMONSTRACĀO - Como a) do exemplo 1.

3) Seja $f:[a, b] \rightarrow L_{\infty}([a, b])$, dada por $f(t)=X_{[a, t]} \cdot \hat{E}$ imediato que:

a) $f \in B W\left([a, b], L_{\infty}([a, b])\right)$;

b) $\operatorname{ftM}\left([a, b], L_{\infty}([a, b])\right)$.

DEMONSTRAC $\bar{A} O$ - Como a do exemp1o 1.

QUESTŌES 3.a.14 - Queremos propor algumas questões sobre a mensurabilidade de funçöes de $B([a, b], Y)$ e $R([a, b], Y)$. 
[Q-1] - Caracterizar os espaços de Banach Y para os quais $R([a, b], Y) \subset M([a, b], Y)$.

[Q-2] - Caracterizar os espaços de Banach Y para os quais $B W([a, b], Y) \subset M([a, b], Y)$.

As questões acima nos parecem muito difíceis, e por isto $\vec{e}$ natural propormos inicialmente as mesmas questões em certas classes particulares de espaços de Banach, tais como:

1) Y fracamente sequencialmente completo (em particular, reflexivo).

2) $Y=X^{\prime}(Y$ um dua 1$)$.

$\underline{\text { PROPOSIÇĀO }}$ 3.a.15 - Seja Y um espaço de Banach que não contém subespaço isomorfo a $C_{0}(\mathbb{N})$. Então $B W([a, b], Y) \subset M([a, b], Y)$. (A mesma demonstração mostra que

$$
\operatorname{SV}([a, b], L(X, Y)) \subset M([a, b], L(X, Y)))
$$

DEMONSTRACÄO - Pelo corolärio 3. a.6 sabemos que, se Y não contém subespaço isomorfo a $C_{0}(\mathbb{N})$ então $B(W([a, b], Y) \subset G([a, b], Y)$, e como as funções regradas são limite uniforme de funções em escada (por 2 da proposição 1.c.2), segue que

$$
G([a, b], Y) \subset M([a, b], Y)
$$

e portanto, $B W([a, b], Y) \subset M([a, b], Y)$.

0 corolärio abaixo responde a questão [Q-2] no caso particular em que $Y \vec{e}$ fracamente sequencialmente completo. 
COROLARIO 3.a.16 - Se Y ë um espaço de Banach fracamente sequencialmente completo (em particular, reflexivo) então

$$
B W([a, b], Y) \subset M([a, b], Y) .
$$

DEMONSTRACĀO - E decorrência imediata da proposição 3.a.15, pois tais espaços não podem conter subespaço isomorfo a $C_{0}(\mathbb{N})$.

CQD

OBSERVAÇĀO 3.a.17 - 0 exemp1o 3.a.13.1 mostra que [Q-1] e [Q-2] são problemas distintos pois, naquele exemplo, temos:

1) $B W([0,1], H) \subset M([0,1], H)$ (veja corolário 3.a.16).

2) $\mathrm{R}([0,1], H) \not \subset M([0,1], H)$ (Exemp 10 3.a.13).

o teorema que se segue resolve a questão [Q-2] no caso em que Y ẻ um espaço dual. Omitimos a demonstração para não alongar em demasia este trabalho.

TEOREMA 3.a.18 - Seja Y um espaço de Banach dual (isto é $Y=$ $\left.=X^{\prime}\right)$. São equivalentes:

1) Y não contẻm subespaço isomorfo a $C_{0}(\mathbb{N})$.

2) Y não contém subespaço isomorfo a $\ell_{\infty}(\mathbb{N})$.

3) $B W([a, b], Y) \subset M([a, b], Y)$.

COROLẢRIO 3.a.19 - 1) Se K é um espaço métrico compacto com cardinal infinito, então $C(K)$ não è dual.

2) Se $K$ è um compacto (Hausdorff) disperso (isto è $K$ não contēm nenhum conjunto perfeito e não vazio) com cardinal 
infinito então $C(K)$ não é um dual.

3) Se $x=L_{p}(u), 1 \leq p \leq \infty$, então são equivalentes:

a) $\mathrm{B} W([\mathrm{a}, \mathrm{b}], \mathrm{X}) \subset \mathrm{M}([\mathrm{a}, \mathrm{b}], \mathrm{X})$;

b) $1 \leq \mathrm{p}<\infty$ ou $\mathrm{L}_{\infty}(\mathrm{u})=\mathbb{K}^{\mathrm{n}}$.

Agora surge uma pergunta natura1: Se Y é um espaço de Banach não separāvel, são equivalentes:

1) $Y$ não contẻm subespaço isomorfo a $C_{0}(\mathbb{N})$;

2) $B W([a, b], Y) \subset M([a, b], Y)$ ?

A resposta a esta questão é trivialmente negativa. Basta considerar $Y=C_{0}(\mathbb{N}) \times X$, onde $X$ é não separäve1 e não contẻm $C_{0}(\mathbb{N})$. (Exemplos: H espaço de Hilbert não separävel, $L_{1}$ (u) não separäveis, etc.) .

De fato, se $f \in B W([a, b], Y)$ então, pela proposição 1.d.4, item 5, b) $\left.\Longleftrightarrow b^{\prime}\right)$, virä:

$$
\begin{aligned}
& \mathrm{P}_{1} \circ \mathrm{f} \in \mathrm{B} \omega\left([\mathrm{a}, \mathrm{b}], \mathrm{c}_{0}(\mathbb{N})\right) \stackrel{3 . \mathrm{a} \cdot 12}{=} \mathrm{P}_{1} \circ \mathrm{f \in M}\left([\mathrm{a}, \mathrm{b}], \mathrm{c}_{0}(\mathbb{N})\right) \\
& \mathrm{P}_{2} \circ \mathrm{f} \in B([\mathrm{a}, \mathrm{b}], \mathrm{x}) \stackrel{3 \cdot \mathrm{a} \cdot 15}{=} \mathrm{P}_{2} \circ \mathrm{f \in M}([\mathrm{a}, \mathrm{b}], \mathrm{x}) .
\end{aligned}
$$

$\log o, \operatorname{f\in M}([a, b], Y)$.

No entanto, a mesma questão, restrita à classe dos espaços $C(K)$ ( $K$ compacto, Hausdorff), nos parece nãotrivial e foi resolvida por

TEOREMA 3.a.20 - Existem espaços de Banach $C(K)=Y$ não se- 
paräveis, tais que:

1) $B W([a, b], Y) \subset M([a, b], Y)$.

2) $Y$ contëm subespaço isomorfo a $C_{0}(\mathbb{N})$.

(Observação - A classe a que se refere o teorema $3 . a .20$ e constituída de espaços Y não duais).

3.b - PROBLEMA TRESS

Vamos agora abordar o problema 3, ou seja: "Se X $\vec{e}$ um espaço de Banach de dimensão infinita, podemos afirmar que $D([a, b], X)$ esta contido propriamente em $R([a, b], X)$ ?"

No capítulo 1 demonstramos os seguintes resultados:

1) $D([a, b], X) \subset R([a, b], x)$ (proposiçäo 1.a.9, item 1);

2) $f \in D([a, b], x)$ se e só se $\left\{\begin{array}{c}1) \operatorname{f\in B}([a, b], X) \\ \text { (teorema 1.b.13) } \\ \text { 2) } u[D(f)]=0 ;\end{array}\right.$

3) Se $f \in R([a, b], X)$ então $f \in B([a, b], X)$ (proposição 1.a.7, item 1).

Os resultados 1,2 e 3 acima motivam a seguinte

DEFINICĀO 3.b.1 - Diz-se que um espaço de Banach $x$ possui. a propriedade de Lebesgue (espaço $P L)$ se $D([a, b], X)=R([a, b], X)$ ou, noutras palavras, se toda função $\mathrm{f}:[\mathrm{a}, \mathrm{b}] \longrightarrow \mathrm{X}$ Riemann integrâve1 tem o conjunto de seus pontos de descontinuidade de medida nula (assim, $f \in R([a, b], x) \Longrightarrow u(D(f))=0$ ). Caso 
conträrio, diz-se que o espaço x não tem propriedade de Le-besgue (espaço NPL).

A proposição que se segue tem por objetivo colocar, na 1 inguagem da definição $3 . b .1$, alguns resultados do capitulo 1 .

PROPOSICĀA $\underline{3 . b \cdot 2}$ - 1) Se X $\vec{e}$ um espaço de dimensãofinita então X $\overrightarrow{\mathrm{e}}$ um espaço PL.

2) Se $Y$ é um subespaço fechado de um espaço PL X então Y $\vec{e}$ um espaço PL.

3) Um espaço que contẻm um subespaço NPL è um espaço NPL.

4) Os espaços PL(NPL) são invariantes por isomorfismo, isto é: se X e Y são espaços de Banach, $J: X \rightarrow Y$ è um isomorfismo e X è PL(NPL) então Y è PL(NPL).

5) $C_{0}(I N) ~ \vec{e}$ um espaço NPL de dimensão infinita. DEMONSTRACĀO - 1) Segue de 2 da proposição 1.a.11.

2) Caso conträrio, existiria $f \in R([a, b], Y) \operatorname{com} u(D(f))>0$ e, como $f \in R([a, b], X)$ e $u(D(f))>0, X$ não seria um espaço PL, o que è absurdo.

3) Demonstração anâloga à de 2 .

4) Segue de 3 da proposição 1.a.7, equivalência a) $\Longleftrightarrow$ b), lembrando que, como $\mathrm{J} \overrightarrow{\mathrm{e}}$ isomorfismo, $\mathrm{D}(\mathrm{J} \circ \mathrm{f})=\mathrm{D}(\mathrm{f})$.

5) Veja exemplo 1.e.2, afirmações 2) e 3). 
COROLARIO 3.b.3 - Todo espaço de Banach que contém um subespaço isomorfo a $C_{0}(\mathbb{N})$ é um espaço NPL.

DEMONSTRAÇĀO - E decorrência imediata de 5,4 e 3 da proposição 3.b.2.

TEOREMA 3.b.4 - Seja X um espaço de Banach uniformemente convexo. São equivalentes as propriedades:

1) X tem dimensão finita.

2) $D([a, b], x)=R([a, b], x)$.

DEMONSTRAÇĀO -1$) \Longrightarrow$ 2) Segue de 2 da proposição 1.a.11.

2) $\Longrightarrow 1$ 1) Suponhamos, por absurdo, que $x$ possua dimensão infinita. Então, por 1 do teorema $2 . a \cdot 11, x$ contêm uma sequência bäsica $\left\{x_{i}\right\}_{i=1}^{\infty} \operatorname{com}\left\|x_{i}\right\|=1$. Seja $Y$ o fecho do espaço gerado por $\left\{x_{i}\right\}_{i=1}^{\infty}$. Então $\left\{x_{i}\right\}_{i=1}^{\infty} \vec{e}$ uma base de $Y$, e por 2, teorema 2.b.2, Y é uniformemente convexo.

Dado um intervalo $[\mathrm{a}, \mathrm{b}] \mathrm{c} \mathbb{R}$, seja $\left\{\mathrm{r}_{\mathrm{i}}\right\}_{i=1}^{\infty}$ uma enumeração dos racionais de $[a, b]$. Definimos $f:[a, b] \rightarrow X$ por:

$$
f(x)=\left\{\begin{array}{l}
0, \text { se } x \notin\left\{r_{i}\right\}_{i=1}^{\infty} \\
x_{i} \text { se } x=r_{i}
\end{array}\right.
$$

E imediato que $D(f)=[a, b]$, e portanto $f \notin D([a, b], X)$ (teorema $1 . b .13)$.

Por outro lado, $f \in R([a, b], x)$ e $\int_{a}^{b} f(x) d x=0$. De $f a-$
to: dada uma divisão $d \in D, d=\left\{a=t_{0}<t_{1}<\ldots<t|d|=b\right\}$, utilizan- 
do-se o resultado 5 do teorema $2 . b .2$, e efetuando-se um cälculo simples, virä, para algum $p$ com $1<p<\infty$ :

$$
\begin{aligned}
\left\|\sum_{i=1}^{|d|} f\left(\varepsilon_{i}\right)\left[t_{i}-t_{i-1}\right]-0\right\| & \leq A\left[\sum_{i=1}^{|d|}\left[t_{i}-t_{i-1}\right]^{p}\right]^{1 / p} \leq \\
& \leq A \sqrt[p]{(\Delta d)^{p-1}} \sqrt[p]{\sum_{i=1}^{|d|}\left[t_{i}-t_{i-1}\right]}=A \sqrt[p]{(\Delta d)^{p-1}} \sqrt{(b-a)}
\end{aligned}
$$

Assim, dado $\varepsilon>0$, se tomarmos $\Delta d \leq \delta=\left[\frac{\varepsilon}{A \sqrt{b-a}}\right]^{p /(p-1)}$, virä que

$$
\left\|\sum_{i=1}^{|d|} f\left(\varepsilon_{i}\right)\left[t_{i}-t_{i-1}\right]-0\right\| \leq \varepsilon \text {, ou seja, } \int_{a}^{b} f(x) d x=0
$$

- que contraria a hipötese.

COROLARIO 3.b.5 - 1) Seja X um espaço de Hilbert. São equivalentes a) e $a^{\prime}$ ):

a) $x$ possui dimensão finita.

$\left.a^{\prime}\right) D([a, b], x)=R([a, b], x)$.

2) Seja $x=L_{p}(u), 1<p<\infty$. São equivalentes a e a':

a) $L_{p}(u)$ possui dimensão finita.

$\left.a^{\prime}\right) D([a, b], X)=R([a, b], X)$.

3) Se $x \vec{e}$ um espaço de Banach uniformemente convexo (em particular, de Hilbert) e de dimensão infinita, então $X$ è um espaço NPL.

4) Se $1<p<\infty$ e $I_{p}(u)$ tem dimensão infinita então $L_{p}(u)$ 
é um espaço NPL.

5) Se $1<p<\infty$ então $L_{p}([a, b], \mathbb{K})$ e $\ell_{p}(\mathbb{N})$ são espaços NPL.

6) Todo espaço de Banach que contẻm um subespaço isomorfo a um espaço uniformemente convexo de dimensão infinita $\vec{e}$ um espaço NPL.

DEMONSTRACĀO - 1) e 2) São decoṛência imediata de 1 e 4 do teorema 2.b.2, e do teorema 3.b.4 anterior.

3) Decorre imediatamente do teorema 3.b.4.

4) Decorre imediatamente de 2 deste corolärio.

5) Decorre de 4) imediatamente, lembrando que $L_{p}([a, b], \mathbb{K})$ e $\iota_{p}(\mathbb{N})$ tem dimensão infinita.

6) Decorre de 3) deste corolärio e de 4 e 3 da proposição $3 \cdot b \cdot 2$.

TEOREMA 3.b.6 - Seja $N$ espaço topológico, Hausdorff e normal (mais geralmente, completamente regular). Denotaremos por $C^{*}(N, \mathbb{K})$ o conjunto das funções contínuas e limitadas $f: N \rightarrow \mathbb{K}$ com a norma $\|f\|=\sup _{x \in N}|f(x)|$. São equivalentes:

1) $N \vec{e}$ um conjunto finito $\left(C^{*}(N, \mathbb{K})=\mathbb{K}^{n}\right)$

2) $C^{*}(N, \mathbb{K}) \vec{e}$ um espaço PL.

DEMONSTRACĀO -1$) \Longrightarrow$ 2) Proposição 1. a.11, item 2 .

$2) \Longrightarrow 1$ ) Por absurdo, suponhamos que $N$ tenha cardinal infinito, e vamos demonstrar que $C^{*}(N, \mathbb{K})$ contém um subespa- 
ço isomorfo a $C_{0}(\mathbb{N})$.

Em primeiro lugar, vamos demonstrar que um espaço de Hausdorff infinito $N$ contêm uma sequência de abertos $\left\{A_{i}\right\}_{i=1}^{\infty}$ dois a dois disjuntos e não vazios.

De fato, como $N$ è de Hausdorff contendo mais de um ponto, existem abertos $A_{1}$ e $A_{2}$ não vazios e disjuntos.

Se um destes abertos ë um conjunto finito denotamos o mesmo por $A_{1}$; e passamos a trabalhar no $C A_{1}$ que $\vec{e}$ um aberto infinito (pois $N \vec{e}$ infinito).

Caso contrário, denotamos um deles por $A_{1}$ e passamos a trabalhar no aberto que restou que $\vec{e}$ tambëm infinito.

Suponhamos construída a coleção de abertos näo va$\operatorname{zios} A_{1}, A_{2}, \ldots, A_{n-1}, A_{n}$, onde $A_{i} \cap A_{j}=\emptyset$, se $i \neq j, A_{i} \cap A_{n}=\varnothing$, $1 \leq i \leq n-1$ e $A_{n} \vec{e}$ um aberto infinito. Tomemos agora em $A_{n}$ dois abertos $A_{1}^{\prime}$ e $A_{2}^{\prime}$ não vazios edisjuntos ( $A_{1}^{\prime}$ e $A_{2}^{\prime}$ säo abertos em N).

Se um deles é finito denotemô-10 por $A_{n}$ e seu complementar em $A_{n}$ por $A_{n+1}$. Se ambos são infinitos, denotamos um deles pora $A_{n}$ e o outro por $A_{n+1}$. Obtemos assim uma coleção de abertos não vazios $A_{1}, A_{2}, \ldots, A_{n-1}, A_{n}, A_{n+1}$ onde $A_{i} \cap A_{j}=\varnothing$, se $i \neq j, A_{1} \cap A_{n+1}=\varnothing, 1 \leq i \leq n$ e $A_{n+1}$ é um aberto infinito. Logo, podemos construir em $N$ a sequência de aber$\operatorname{tos}\left\{A_{i}\right\}_{i=1}^{\infty}$ dois a dois disjuntos e não vazios.

Consideremos os fechados $F_{i}=\left\{\mathrm{p}_{i}\right\} \subset \mathrm{A}_{i}$ e $\mathrm{F}_{i}^{\prime}=\mathrm{CA}_{i} \cdot \mathrm{Co}-$ 
mo $N$ e completamente regular existem $f_{i}: N \rightarrow[0,1]$ contínuas e tais que

$$
\begin{aligned}
& f_{i}(x)=\left\{\begin{array}{l}
1, \text { se } x=p_{i}, 0 \leq f_{i}(x) \leq 1 \\
0 \text { se } x \in C A_{i} .
\end{array}\right. \\
& \text { Se } e_{i}=(0,0, \ldots, i, 0,0,0, \ldots) \in C_{0}(\mathbb{N}) \text {, definimos } \\
& \mathrm{T}: \mathrm{C}_{0}(\mathbb{N}) \rightarrow C^{*}(N, \mathbb{K})
\end{aligned}
$$

como a extensão linear contínua de $\mathrm{T}\left(\mathrm{e}_{i}\right)=\mathrm{f}_{i} \cdot$ Se

$$
x=\sum_{i=1}^{m} a_{i} e_{i} \text {, vem que }\|T(x)\|=\left\|\sum_{i=1}^{m} a_{i} f_{i}\right\|=\max _{1 \leq i \leq m}\left|a_{i}\right|
$$

e portanto, a função em questão tem extensão linear que $\vec{e} u-$ ma isometria entre $C_{0}(\mathbb{N})$ e o fecho do subespaço gerado por $\left\{f_{i}\right\}_{i=1}^{\infty}$

Se $N$ é um espaço de Hausdorff, completamente regular (em particular, normal), de cardinal infinito, demonstramos que $C^{*}(N, \mathbb{K})$ contẻm subespaço isomorfo a $C_{0}(\mathbb{N})$, e segue do corolärio $3 . b .3$ que $C^{*}(N, \mathbb{K})$ è um espaço NPL, o que contraria a hipötese. CQD

COROLARIO $3 . \mathrm{b} .7$ - 1) Seja $N$ um espaço de Hausdorff e normal (completamente regular). São equivalentes:

a) $N$ è um conjunto finito.

$\left.a^{\prime}\right) C^{*}(N, \mathbb{K})$ é um espaço PL.

a") $C^{*}(N, \mathbb{K})$ è reflexivo. 
$\left.a^{\prime \prime \prime}\right) C^{*}(N, \mathbb{K})$ possui dimensão finita.

2) Seja K um espaço compacto. São equivalentes:

a) $K \vec{e}$ finito

$\left.a^{\prime}\right) \mathcal{C}(K)$ é um espaço PL.

$\left.\mathrm{a}^{\prime \prime}\right)(\mathrm{K})$ è reflexivo.

$\left.a^{\prime \prime \prime}\right) C(K)$ possui dimensão finita.

3) Seja K um espaço compacto e de cardinalidade infinita. Então valem as seguintes propriedades equivalentes:

a) $C(K)$ é um espaço NPL.

b) $C(K)$ não é reflexivo.

DEMONSTRACA $\bar{A} O$ - 1) Imediato a partir do teorema $3 . b .6$.

2) Imediato a partir do teorema 3.b.6, lembrando que todo compacto $K$ ë Hausdorff, normal e $\mathcal{C}^{*}(K, \mathbb{K})=\mathcal{C}(K, \mathbb{K})=\mathcal{C}(K)$.

3) Consequência imediata do 2) C CQD COROLARro 3.b.8 - 1) Seja $X=L_{\infty}(u)$. São equivalentes:

a) $L_{\infty}(u)$ tem dimensão finita.

$\left.a^{\prime}\right) L_{\infty}(u)$ é um espaço PL.

$a^{\prime \prime)} L_{\infty}(u)$ è reflexivo.

2) Se $L_{\infty}(u)$ possui dimensão infinita então $\vec{e}$ um espaço NPL .

3) $L_{\infty}([a, b], \mathbb{K}) e \ell_{\infty}(\mathbb{N})$ são espaços NPL. 
DEMONSTRACĀO - 1) E imediato que a) $\Longrightarrow a^{\prime}$ ) e a) $\Longrightarrow a^{\prime \prime}$ ). Do teorema $2 . b .3$, sabemos que $L_{\infty}(u)$ é isomorfo a $C(K)$, para a 1 gum conjunto $K$ compacto de Hausdorff. Se negamos a), é claro que $K$ serả infinito: entäo $C(K)$ e, consequentemente, $L_{\infty}(u)$, possuirão subespaços isomorfos a $C_{0}(u)$. Logo, $L_{\infty}(u)$ não pode ser PL nem reflexivo, efica demonstrado 1).

2) $\vec{E}$ consequência imediata de 1).

3) E consequência imediata de 2). CQD

A proposição que segue termina a classificação dos espaços $L_{p}([a, b], \mathbb{K}), l \leq p \leq c$. Mais tarde (corolärio 3.b.14), obtemos novamente tal resultado.

$\underline{\text { PROPOSICÄO } 3 . b .9}-L_{1}([a, b], \mathbb{K})$ è um espaço NPL.

DEMONSTRACĀO - Basta provar que $L_{1}([a, b]$, IK) contëm um subespaço isomorfo a um espaço de Hilbert de dimensão infinita (veja 6 do corolärio $3 \cdot b, 5)$. Faremos a demonstração para $[0,1]$. Para isto considere-se $J: \ell_{2}(\mathbb{N}) \rightarrow L_{1}([0,1], \mathbb{K})$, dada por

$$
J\left(a_{1}, a_{2}, \ldots, a_{n}, \ldots\right)(t)=\sum_{n=1}^{\infty} a_{n} r_{n}(t),
$$

onde $r_{n}(t)$ são as funçöes de Rademacher, definidas na proposição 2.b.4. Pela mesma proposição virä, para o caso de $\mathrm{p}=1$, que

$$
A(1)\left\|\left(a_{1}, a_{2}, \ldots, a_{m}, 0,0, \ldots\right)\right\|_{2} \leq \int_{0}^{1}\left|\sum_{n=1}^{m} a_{n}{ }^{r}{ }_{n}(t)\right| d t \leq
$$




$$
\leq B(1)\left\|\left(a_{1}, a_{2}, \ldots, a_{m}, 0,0, \ldots\right)\right\|_{2},
$$

ou seja, no espaço gerado pelos $e_{i}=(0 \ldots 0$ i $00 \ldots)$, temos: $A(1)\|x\|_{2} \leq\|J(x)\|_{1} \leq B(1)\|x\|_{2}$, e portanto, em $\ell_{2}(\mathbb{N}),(1$ embrando que $L_{1}([0,1], \mathbb{K})$ e completo), $A(1)\|x\|_{2} \leq\|J(x)\| \leq B(1)\|x\|_{2}$. Logo, J está bem definida e $\vec{e}$ um isomorfismo linear sobre sua imagem.

COROLÅRIO $3 . b \cdot 10-L_{p}([a, b], \mathbb{K})$ è um espaço NPL, $1 \leq p \leq \infty$. DEMONSTRAÇĀO - $\widehat{E}$ consequência das proposições $3 . \mathrm{b} .9,3$ do corolärio 3. b.8, e 5 do corolärio $3 . b .5$.

OBSERVAÇĀO 3.b.11 - Usando o mesmo raciocínio da proposição 3.b.9, podemos provar que, em $L_{p}([a, b], \mathbb{K}), 1 \leq p<\infty$, existe um subespaço isomorfo a um espaço de Hilbert de dimensão infinita (precisamente o $\left.\ell_{2}(\mathbb{N})\right)$ e, com isto, obtemos uma outra demonstração de que estes espaços são espaços NPL (veja 5 do corolärio $3 . b .5$ ).

Surge naturalmente a seguinte questão: "Todo espaço de Banach de dimensão infinita é um espaço NPL?" Antes de terminarmos a classificação dos espaços de medida $L_{p}(u)$, $1 \leq \mathrm{p} \leq \infty$, vamos mostrar alguns exemplos de espaços de dimensão infinita com a propriedade de Lebesgue.

TEOREMA $\underline{3 . b .12}-\ell_{1}(\mathbb{N})$ ë um espaço com a propriedade de Lebesgue (espaço PL).

DEMONSTRACĀO - Vamos provar que, se $\operatorname{fED}\left([\mathrm{a}, \mathrm{b}], \mathrm{l}_{1}(\mathbb{N})\right)$ então 
$f \notin R\left([a, b], \ell_{1}(\mathbb{N})\right)$. Se $f \ell D\left([a, b], \ell_{1}(\mathbb{N})\right)$ então temos duas possibilidades:

1) $f \notin B\left([a, b], \ell_{1}(\mathbb{N})\right)$; nestas condições (proposição 1.a.7, item 1) sabemos que $f \notin R\left([a, b], \ell_{1}(\mathbb{N})\right)$.

2) $f \in B\left([a, b], \ell_{1}(\mathbb{N})\right)$ e $u(D(f))>0$. Neste caso como

$$
D(f)=\bigcup_{n=1}^{\infty} F_{n}, \operatorname{com} F_{n}=\left\{t \mid \omega(t, f) \geq \frac{1}{n}\right\}
$$

existe $u m$ indice $n_{0}$ tal que $u\left[\mathrm{~F}_{\mathrm{n}_{0}}\right]=\mathrm{C}>0$.

Sejam as funções $P_{n}: \ell_{1}(\mathbb{N}) \rightarrow \mathbb{K}$, dados por $P_{n}(x)=$ $=x_{n}$, para todo $x \in l_{1}(\mathbb{N})$ (projeções). Se feR $\left([a, b], l_{1}(\mathbb{N})\right)$, podemos afirmar (por 2 ,proposição 1.a.7) que

$$
P_{n} \circ f \in R([a, b], \mathbb{K})=D([a, b], \mathbb{K})
$$

Da proposição 1.b.13, virä que $u\left(D\left(p_{n} \circ f\right)\right)=0$, para todon $\in \mathbb{N}$; $\log \mathrm{O}$

$$
u\left[\bigcup_{n=1}^{\infty} D\left(P_{n} \circ f\right)\right]=0
$$

Tomemos uma divisão $d=\left\{a=t_{0}<t_{1}<\ldots<t_{K}=b\right\}$ de $[a, b] c o m$

$$
t_{i}-t_{i-1}=\frac{b-a}{k} \text {, para } 1 \leq i \leq k \text {, }
$$

e consideremos os intervalos desta divisão que interceptam $\mathrm{F}_{0}$ em um conjunto de medida não nula; vamos denotar estes intervalos por $\dot{I}_{1}, I_{2}, \ldots, I_{p}$. Para todo $i, I \leq i \leq p$, existe $x \in I_{i}$, com 


$$
x \in F_{n_{0}} \text { e } x \notin \bigcup_{n=1}^{\infty} D\left(P_{n} \circ f\right) \text {, ou seja, } x \in F_{n_{0}}{ }^{\circ} I_{i} \text {. }
$$

Mas para todo n natural, $\mathrm{P}_{\mathrm{n}}$ of é contínua em $\mathrm{x}$. Observemos ainda que

$$
\frac{p(b-a)}{K} \geq C(*) \text { pois } u\left(F_{n_{0}}\right)=C \text { e } F_{n_{0}} \subset \bigcup_{i=1}^{p} I_{i}
$$

(a menos de um conjunto de medida nula). Escolhemos

$$
x_{1} \in \stackrel{O}{I}{ }_{1}^{n F_{n_{0}}}, x_{1} \notin \bigcup_{n=1}^{\infty} D\left(P_{n} \circ f\right):
$$

existe $y_{1} \in I_{1}$ ta1 que $\left\|f\left(x_{1}\right)-f\left(y_{1}\right)\right\| \geq \frac{1}{2 n_{0}}$. Seja

$$
e_{1}=f\left(x_{1}\right)-f\left(y_{1}\right)=\left(a_{1}^{1}, a_{2}^{1}, a_{3}^{1}, \ldots, a_{j}^{1}, \ldots\right) .
$$

Dado $\varepsilon>0$, tomemos $j_{1}$ tal que

$\sum_{i=j_{1}}^{\infty}\left|a_{i}^{1}\right|<\frac{\varepsilon}{2^{20}}$. Escolhemos $x_{2} \in I_{2}^{\circ} \cap F_{n_{0}}, x_{2} \notin \bigcup_{n=1}^{\infty} D(P \circ o f)$ e $y_{2} \in I_{2}$

com as seguintes propriedades:

a) $\left\|f\left(x_{2}\right)-f\left(y_{2}\right)\right\| \geq \frac{1}{2 n_{0}}$;

b) $\sum_{i=1}^{j_{1}}\left|\left(P_{i} \circ f\right)\left(x_{2}\right)-\left(P_{i} \circ f\right)\left(y_{2}\right)\right|<\frac{\varepsilon}{2^{21}}$ (Isto é possível, pois $\left.x_{2} \notin \bigcup_{n=1}^{\infty} D\left(P_{n} \circ f\right)\right)$.

Seja $e_{2}=f\left(x_{2}\right)-f\left(y_{2}\right)=\left(a_{1}^{2}, a_{2}^{2}, \ldots, a_{j}^{2}, \ldots\right)$. Consideremos $j_{2}$ tal que $j_{2}>j_{1}$ e $\sum_{i=j_{2}}^{\infty}\left|a_{i}^{2}\right|<\frac{\varepsilon}{222}$. 
Em $I_{3}$, tomemos

$$
\mathrm{x}_{3} \in \mathrm{I}_{3} \cap \mathrm{F}_{\mathrm{n}_{0}}, \mathrm{x}_{3} \notin \prod_{\mathrm{n}=1}^{\infty} \mathrm{D}\left(\mathrm{P}_{\mathrm{n}} \text { 。f) e } \mathrm{y}_{3} \in \mathrm{I}_{3}\right.
$$

com as seguintes propriedades:

a) $\left\|f\left(x_{3}\right)-f\left(y_{3}\right)\right\| \geq \frac{1}{2 n_{0}}$

b) $\sum_{i=1}^{j_{2}}\left|\left(P_{i} \circ f\right)\left(x_{3}\right)-\left(P_{i} \circ f\right)\left(y_{3}\right)\right| \leq \frac{\varepsilon}{2^{23}}$.

Seja $e_{3}=f\left(x_{3}\right)-f\left(y_{3}\right)=\left(a_{1}^{3} \ldots a_{j}^{3} \ldots\right)$. Escolhemos $j_{3}$ tal que

$$
j_{3}>j_{2} \text { e } \sum_{i=j_{3}}^{\infty}\left|a_{i}^{3}\right|<\frac{\varepsilon}{224} \text {. }
$$

Prosseguimos analogamente nos restantes intervalos, $\mathrm{I}_{4}, \mathrm{I}_{5}$, . $\ldots, I_{p}$, e encontramos vetores

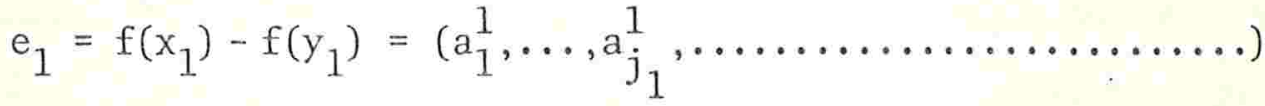

$$
\begin{aligned}
& e_{2}=f\left(x_{2}\right)-f\left(y_{2}\right)=\left(a_{1}^{2}, \ldots, a_{j_{1}}^{2}, \ldots, a_{j_{2}}^{2} \ldots \ldots . . . . . . . .\right) \\
& e_{3}=f\left(x_{3}\right)-f\left(y_{3}\right)=\left(a_{1}^{3}, \ldots, a_{j_{1}}^{3}, \ldots, a_{j_{2}}^{3}, \ldots, a_{j_{3}}^{3} \ldots . . . . .\right) \\
& \vdots \quad \vdots \quad \vdots \\
& e_{p}=f\left(x_{p}\right)-f\left(y_{p}\right)=\left(a_{1}^{p}, \ldots, a_{j_{1}}^{p}, \ldots, a_{j_{2}}^{p}, \ldots, a_{j_{3}}^{p}, \ldots, a_{j_{p}}^{p}, \ldots\right)
\end{aligned}
$$

Então, definindo por 


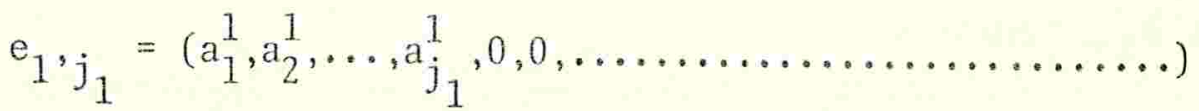

$$
\begin{aligned}
& e_{2}, j_{2}=\left(0,0, \ldots, 0, a_{j_{1}+1}^{2}, a_{j_{1}+2}^{2}, \ldots, a_{j_{2}}^{2}, 0,0, \ldots \ldots \ldots . .\right) \\
& e_{p}{ }^{\prime} j_{p}=\left(0,0, \ldots, 0,0,0, \ldots, 0,0, \ldots, a_{j}^{p}{ }_{p-1}+1, \ldots, a_{j_{p}}^{p}, 0, \ldots\right) \text {, }
\end{aligned}
$$

temos:

$$
\begin{aligned}
& \left\|\sum_{i=1}^{p} e_{i}\right\| \geq\left\|\sum_{i=1}^{p} e_{i, j}\right\|-\left\|\sum_{i=1}^{p}\left(e_{i}-e_{i, j}\right)\right\| \geq\left(\frac{1}{2 n_{0}}-\frac{\varepsilon}{2^{20}}\right)+ \\
& +\left(\frac{1}{2 n_{0}}-\frac{\varepsilon}{2^{21}}-\frac{\varepsilon}{2}\right)+\ldots\left(\frac{1}{2 n_{0}}-\frac{\varepsilon}{\left.2^{20+2 p-3}-\frac{\varepsilon}{2^{20+2 p-2}}\right)-}\right. \\
& -\left\|\sum_{i=1}^{p}\left[e_{i}-e_{i, j}\right]\right\| \geq \frac{p}{2 n_{0}}-2\left[\frac{\varepsilon}{2^{20}}+\ldots+\frac{\varepsilon}{\left.2^{20+2 p-2}\right] \geq}\right. \\
& \geq \frac{p}{2 n_{0}}-2 \frac{2^{20}}{1-\frac{1}{2}}>\frac{p}{2 n_{0}}-\frac{\varepsilon}{2} .
\end{aligned}
$$

Retomemos a divisão $d=\left\{a=t_{0}<t_{1}<\ldots<t_{K}=b\right\}$ e façamos

$$
\left.\begin{array}{l}
\varepsilon_{i}=x_{j} \\
\varepsilon_{i}^{\prime}=y_{j}
\end{array}\right\} \sec \left[t_{i-1}, t_{i}\right]=I_{j}, 1 \leq j \leq p ;
$$

$\varepsilon_{i}=\varepsilon_{i}^{\prime}$ se $\left[t_{i-1}, t_{i}\right] \neq I_{j}$, para todo $j$ com $1 \leq j \leq p$.

Desta forma, obtemos, para a soma de Riemann correspondente à d e à escolha dos $\varepsilon_{i}^{\prime} s$, que:

$$
\left\|\sum_{i=1}^{|d|}\left[f\left(\varepsilon_{i}\right)-f\left(\varepsilon_{i}^{\prime}\right)\right] \frac{b-a}{k}\right\|=\left\|\sum_{\varepsilon_{i}^{\prime}, \varepsilon_{i} \in I_{i}, 1 \leq i \leq p}\left[f\left(\varepsilon_{i}\right)-f\left(\varepsilon_{i}^{\prime}\right)\right] \frac{b-a}{K}\right\|=
$$




$$
\begin{aligned}
& \quad=\left(\frac{b-a}{K}\right)\left\|e_{1}+e_{2}+\ldots+e_{p}\right\| \geq\left(\frac{b-a}{K}\right)\left(\frac{p}{2 n_{0}}-\frac{\varepsilon}{2}\right)=\frac{p(b-a)}{2 K n_{0}}-\frac{\varepsilon}{2 K}(b-a) \geq \\
& \stackrel{(*)}{\geq} \frac{C}{2 n_{0}}-\frac{\varepsilon(b-a)}{2} . \\
& \text { Tomando } \varepsilon<\frac{\varepsilon}{2 n_{0}(b-a)}, \text { vira }: \\
& \left\|\sum_{i=1}^{|d|}\left[f\left(\varepsilon_{i}\right)-f\left(\varepsilon_{i}^{\prime}\right)\right] \frac{b-a}{K}\right\| \geq \frac{C}{2 n_{0}}-\frac{C(b-a)}{4 n_{0}(b-a)} \geq \frac{C}{4 n_{0}}>0 .
\end{aligned}
$$

Pela proposição $1 . a .5$, temos que $f \mathscr{l} R\left([a, b], \ell_{1}(\mathbb{N})\right)$, o que termina a demonstração.

$\underline{\text { TEOREMA }} \underline{3 . b .13}$ - Seja $\Gamma$ um conjunto não vazio, $\mathbb{K}=\mathbb{R}$ ou $\mathbb{C}$. 0 espaço de Banach $\ell_{1}(\Gamma, \mathbb{K})=\left\{f: \Gamma \rightarrow \mathbb{K}\left|\sum_{x \in \Gamma}\right| f(x) \mid<\infty\right\}$, com a norma $\|f\|=\sum_{x \in \Gamma}|f(x)|$, possui a propriedade de Lebesgue.

DEMONSTRAÇĀO - Se $\Gamma$ è finito ou enumerävel, o teorema é consequência de 2 da proposição $1 . a .11$ e doteorema 3.b.12. Resta demonsträ-1o, pois, para $\Gamma$ não enumeräve1. A demonstração segue a mesma linha do teorema 3.b.11. Se

$$
f \in R\left([a, b], \ell_{1}(\Gamma, \mathbb{K})\right) \quad \text { e } \quad f \in D\left([a, b], \ell_{1}(\Gamma, \mathbb{K})\right)
$$

então $u(D(f))>0$ e, como $D(f)=\bigcup_{n=1}^{\infty} F_{n}$, existe $n_{0} \in \mathbb{N}$ tal que $\mathrm{u}\left(\mathrm{F}_{\mathrm{n}_{0}}\right)=\mathrm{C}>0$.

Dada a divisão $d=\left\{a=t_{0}<t_{1}<\ldots<t_{K}=b\right\}, c$ com $t_{i}{ }^{-t} t_{i-1}=$ $=\frac{\mathrm{b}-\mathrm{a}}{\mathrm{K}}, 1 \leq \mathrm{i} \leq \mathrm{K}$, denotemos por $\mathrm{I}_{1}, \ldots, \mathrm{I}_{\mathrm{p}}$ os intervalos de $\mathrm{d}$ que interceptam $\mathrm{F}_{\mathrm{n}_{0}}$ num conjunto de medida não nula. 
Seja $x_{1} \in I_{1} \cap F_{n_{0}}$; existe $y_{1} \in I_{1}$ tal que, para $e_{1}=$ $=f\left(x_{1}\right)-f\left(y_{1}\right)$, vale que $\left\|f\left(x_{1}\right)-f\left(y_{1}\right)\right\| \geq \frac{1}{2 n_{0}}$. Por sua vez, $e_{1} \in \ell_{1}(\Gamma, \mathbb{K})$, e portanto $e_{1}$ possui apenas um conjunto enumerävel de coordenadas diferentes de zero; denotemos por $i_{1}^{1}$, $i_{2}^{1}, \ldots, i_{n}^{1}, \ldots$ os indices onde $e_{1} ; \Gamma \rightarrow \mathbb{K}$ possui tais coordenadas. Tomemos $i_{J_{1}}^{1}$ tạl que

$$
\sum_{n=J_{1}}^{\infty}\left|\ell_{1}\left(a_{i_{n}}\right)\right|<\frac{\varepsilon}{2^{20}}
$$

Definimos $P_{j}: \ell_{1}(\Gamma, \mathbb{K}) \rightarrow \mathbb{K}$ por $P_{j}\left[\left(a_{i}\right)_{i \in \Gamma}\right]=a_{j}$, para todo jeI. Se $f \in R\left([a, b], \ell_{1}(\Gamma, \mathbb{K})\right)$ então $P_{i_{n}}{ }^{\circ} f \in R([a, b], \mathbb{K})$, para todo $n \in \mathbb{N}$, e

$$
u\left[\bigcup_{n=1}^{\infty} D\left(P, i_{n}^{1} \circ\right)\right]=0
$$

Em consequência, existe $x_{2} \in I_{2}{ }^{n F} n_{0}, x_{2} \in \bigcup_{n=1}^{\infty} D\left(P i_{n}{ }^{\circ f}\right)$ : podemos entäo encontrar $y_{2} \in I_{2}$ tal que, para $e_{2}=f\left(x_{2}\right)-f\left(y_{2}\right)$, temos:

a) $\left\|f\left(x_{2}\right)-f\left(y_{2}\right)\right\| \geq \frac{1}{2 n_{0}}\left(\right.$ o que é possível, pois $\left.x_{2} \in F_{n_{0}}\right)$. $i^{i}{ }_{1}^{1}$

b) $\sum\left|\left(P_{i}{ }_{n} \circ f\right)\left(x_{2}\right)-\left(P_{i}{ }_{n} \circ f\right)\left(y_{2}\right)\right| \leq \frac{\varepsilon}{2^{2 I}}$.

Denotemos por $i_{1}^{2}, i_{2}^{2}, i_{3}^{2}, \ldots, i_{n}^{2}, \ldots$ os indices em que $e_{2}$ não se anula: seja $i_{J_{2}}^{2}$ tal que

$$
\sum_{n=J_{2}}^{\infty}\left|a_{i_{n}^{2}}\right|<\frac{\varepsilon}{2^{22}}, j_{2}>j_{1}
$$


(se esta desigualdade tem sentido).

Em $I_{3}$, tomemos $x_{3}$ e $F_{n_{0}} n_{3}^{\circ}$ e usando as projeções $\mathrm{P}_{\mathrm{i}_{1}}^{1}, \mathrm{P}_{\mathrm{i}_{2}}^{1}, \ldots, \mathrm{P}_{\mathrm{i}_{1}}^{1}, \mathrm{P}_{\mathrm{i}_{1}}^{2}, \mathrm{P}_{\mathrm{i}_{2}}^{2}, \ldots, \mathrm{P}_{\mathrm{i}_{\mathrm{J}_{2}}}^{2}$, passemos a escolher $y_{3}$ ta 1 que se $e_{3}=f\left(x_{3}\right)-f\left(y_{3}\right)$ então:

a) $\left\|f\left(x_{3}\right)-f\left(y_{3}\right)\right\| \geq \frac{1}{2 n_{0}}$;

b) $\sum_{i=1}^{J_{1}}\left|\left(P i_{n}^{1} \circ f\right)\left(x_{3}\right)-\left(P i_{n}^{1} \circ f\right)\left(y_{3}\right)\right|+\sum_{i=1}^{J} \mid\left(P i_{n}^{2} \circ f\right)\left(x_{3}\right)-$ $-\left(P i_{n}^{2} \circ\right)\left(y_{3}\right) \mid \leq \frac{\varepsilon}{2^{23}}$

Procedendo de modo anälogo em $I_{4}, I_{5}, \ldots, I_{p}$, e utilizando o mesmo método do teorema $3 . b .12$ chegamos a

$$
\left|\sum_{i=1}^{|d|}\left[f\left(\varepsilon_{i}\right)-f\left(\varepsilon_{i}^{\prime}\right)\right] \frac{b-a}{k}\right| \geq \frac{c}{4 n_{0}}
$$

o que contraria a proposiçäo 1.a.5. Logo, $f \notin R\left([a, b], \ell_{1}(\Gamma, \mathbb{K})\right)$.

CQD

O teorema seguinte mostra como obter novos espaços com a propriedade de Lebesgue a partir de espaços que têm esta propriedade.

TEOREMA $3 \cdot$ b.14 - Seja $\left\{x_{n}\right\}_{n=1}^{\infty}$ uma sequência de espaços com a propriedade de Lebesgue. Então:

$$
x=\oplus\left(\sum_{n=1}^{\infty} x_{n}\right)_{1}=\left\{x=\left(x_{1}, x_{2}, \ldots, x_{n}, \ldots\right) \mid x_{n} \in X_{n},\|x\|=\sum_{i=1}^{\infty}\left\|x_{i}\right\|<\infty\right\}
$$


è um espaço com a propriedade de Lebesgue.

DEMONSTRAÇĀO - A demonstração segue a mesma linha dos teoremas $3 . b .12$ e 3.b.13. Se $f \notin D([a, b], X)$ e $f \in R([a, b], x)$ então existe $\mathrm{F}_{\mathrm{n}_{0}}$ tal que $\mathrm{u}\left[\mathrm{F}_{\mathrm{n}_{0}}\right]=\mathrm{C}>0$. Dada a divisão $\mathrm{d}=\left\{\mathrm{a}=\mathrm{t}_{0}<\right.$ $\left.<t_{1}<\ldots<t_{K}=b\right\}, \quad$ com $t_{i}-t_{i-1}=\frac{b-a}{K}, 1 \leq i \leq K$, denotemos por $I_{1}$, $\ldots, I_{p}$ os intervalos de $d$ que interceptam $F_{n_{0}}$ num conjunto de medida não nula. Consideremos agora $P_{n}: x \rightarrow x_{n}$ onde $\mathrm{P}_{\mathrm{n}}\left(\mathrm{x}_{1}, \ldots, \mathrm{x}_{\mathrm{n}}, \ldots\right)=\mathrm{x}_{\mathrm{n}}$, que são funções 1 ineares e contínuas. Vale que (por 2 da proposição 1.a.7) $P_{n} \circ f \in R\left([a, b], x_{n}\right)$ e,como $X_{n} \vec{e}$ um espaço PL, temos que $u\left[D\left(P_{n} \circ f\right)\right]=0$. Logo,

$$
u\left[\bigcup_{n=1}^{\infty} D\left(P_{n} \circ f\right)\right]=0
$$

Seja $x_{1} \in I_{1} \cap F_{n_{0}}$; existe $y_{1} \in I_{1}$ tal que, se $e_{1}=f\left(x_{1}\right)-f\left(y_{1}\right)$ então $\left\|f\left(x_{1}\right)-f\left(y_{1}\right)\right\| \geq \frac{1}{2 n_{0}}$. Denotemos

$$
e_{1}=f\left(x_{1}\right)-f\left(y_{1}\right)=\left(a_{1}^{1}, a_{2}^{1}, \ldots, a_{j}^{1}, \ldots\right), a_{j}^{1} \in x_{j},
$$

para todo $j \in \mathbb{N}$, e tomemos $j_{1}$ tal que

$$
\sum_{i=j}^{\infty}\left\|a_{i}^{1}\right\|<\frac{\varepsilon}{2^{20}} .
$$

Vamos agora a $I_{2}$, e consideremos $x_{2} \in I_{2} \cap F_{n_{0}}, x_{2} \notin \bigcup_{n=1}^{\infty} D\left(P_{n} \circ f\right)$ e $y_{2} \in I_{2}$ com as seguintes propriedades:

a) $\left\|f\left(x_{2}\right)-f\left(y_{2}\right)\right\| \geq \frac{1}{2 n_{0}}$;

b) $\sum_{i=1}^{j_{1}}\left\|\left(P_{i} \circ f\right)\left(x_{2}\right)-\left(P_{i} \circ f\right)\left(y_{2}\right)\right\|<\frac{\varepsilon}{2} 2^{21} \quad\left(x_{2} \bigcup_{n=1}^{\infty} D\left(P_{n} \circ f\right)\right)$. 
Se $e_{2}=f\left(x_{2}\right)-f\left(y_{2}\right)=\left(a_{1}^{2}, a_{2}^{2}, \ldots, a_{j}^{2}, \ldots\right), a_{j}^{2} \in x_{j}$, para todo $j$; podemos tomar $j_{2}$ tal que $j_{2}>j_{1}$ e

$$
\sum_{i=j_{2}}^{\infty}\left\|a_{i}^{2}\right\|<\frac{\varepsilon}{2^{22}}
$$

e assim por diante. Como no teorema $3 . b .12$, obtemos que

$$
\operatorname{fER}([\mathrm{a}, \mathrm{b}], \mathrm{X}) \text { CQD }
$$

Estamos prontos para terminar a classificação dos espaços de medida $L_{p}(u), 1 \leq p \leq \infty$. Esta classificação è possível devido ao teorema $3 . b .13$, como veremos a seguir.

TEOREMA 3. b.15 - Se X é um espaço $L_{p}$-abstrato, $1 \leq p \leq \infty$, então são equivalentes:

1) $L_{p}$ possui a propriedade de Lebesgue.

2) $L_{p} \vec{e}$ isomorfo a $\ell_{1}(\Gamma, \mathbb{K})$, para algum conjunto $\Gamma(\mathbb{K}=\mathbb{R}$ ou $\mathrm{C})$.

DEMONSTRAC $\vec{A} O-1) \Longrightarrow$ 2) Por 1 do teorema 2.b.6, X $\vec{e}$ isomorfo a $\left[\ell_{p}(\Gamma, \mathbb{L}) \oplus\left(\oplus \sum_{\alpha \in A} L_{p}\left([0,1]^{m} \alpha, \mathbb{C}\right)\right)\right]_{p}$. Mas, por 2 do teorema 2.b.6, X não pode ter a componente (® $\left.\sum_{\alpha \in A} L_{p}\left([0,1]^{m_{\alpha}}, \mathbb{C}\right)\right)$ p pois senão seria um espaço NPL (esta componente é NPL por conter um subespaço isomorfo a um espaço de Hilbert de dimensão infinita). Concluímos que $L_{p}$ é isomorfo a $\ell_{p}(\Gamma, \mathbb{K})$. Temos agora duas possibilidades a considerar:

1) $\Gamma \vec{e}$ finito. Neste caso, $\ell_{p}(\Gamma, \mathbb{K}) \vec{e}$ isomorfo a $\ell_{1}(\Gamma, \mathbb{K})$. 
2) $\Gamma \vec{e}$ infinito. Então ë imediato que $p=1$, pois senão $\ell_{p}(\mathbb{N})$ seria isomorfo a um subespaço de $\ell_{p}(\Gamma, \mathbb{K})$ e, como $\ell_{p}(\mathbb{N})$, $1<\mathrm{p} \leq \infty$, é um NPL (por 5 do corolärio $3 . b .5$, e por 3 do corolärio $3 . b .8$ ), viria que $X \vec{e}$ NPL; absurdo.

2) $\Longrightarrow 1)$ Se $x$ é isomorfo a $\ell_{1}(\Gamma, \mathbb{K})$ então, pelo teorema 3.b.13, e 4 da proposição $3 . b .2, x$ possui a propriedade de Lebesgue.

CQD

$\underline{\text { COROLĀRIO }} \underline{3 \cdot \mathrm{b} \cdot 16}$ - Seja $X=\mathrm{L}_{\mathrm{p}}(\mathrm{u})$. São equivalentes:

a) $L_{p}(u)$ possui a propriedade de Lebesgue.

b) $L_{p}(u) \vec{e}$ isomorfo a $\ell_{1}(\Gamma, \mathbb{K})$, para algum conjunto $\Gamma$.

DEMONSTRAÇĀO - Imediata a partir do teorema 3.b.15. CQD

o leitor deve observar que o corolário $3 . b .16$ contëm, em particular, a proposição 3.b.9. Entretanto, a primeira demonstração ê muito mais simples.

o teorema que se segue classifica certos duais importantes em anälise.

TEOREMA 3.b.17 - 1) Seja X um espaço de Banach sobre o corpo dos reais que contém um subespaço isomorfo a $\ell_{1}(\mathbb{N})$. Então $X^{\prime}$ è um espaço NPL.

2) Seja K um espaço topológico compacto. São equivalentes as propriedades:

a) K é disperso.

b) $[C(K, \mathbb{R})]^{\prime}$ é isomorfo a $\ell_{1}(\Gamma)$. 
c) $[\mathrm{C}(\mathrm{K}, \mathbb{R})]^{\prime} \vec{e}$ um espaço com a propriedade de Lebesgue. DEMONSTRACÄO -- 1) Por 1 do teorema $2 . b .7$, sabemos que $X^{\prime}$ contẻm subespaço isomorfo a $L_{1}\left(\{0,1\}^{\mathbb{N}}\right)$. Aplicando 2 do teorema $2 . b .7$, vem que $X^{\prime}$ contêm subespaço isomorfo a $L_{1}\left([0,1]^{\mathbb{N}}\right)$, e por 2 do teorema $2 . b, 6$, concluímos que $x^{\prime}$ contếm subespaço isomorfo a $\ell_{2}(\mathbb{N}) ; \operatorname{logo}$, é NPL.

2) a) $\Longrightarrow$ b) E 02 do teorema $2 . b .7$.

b) $\Longrightarrow$ c) Consequência imediata do teorema $3 . b, 13$.

c) $\Longrightarrow$ a) Senão, por 3 do teorema $2 . b .7,[C(K)]^{\prime}$ conteria $L_{1}\left([0,1]^{\mathbb{N}}\right)$, e por 2 do teorema $2 . b .6$, seria NPL, o que contraria a hipôtese.

CQD

COROLARIO 3.b.18 - 1) Seja X um espaço de Banach que contëm um subespaço isomorfo a $\ell_{1}(\mathbb{N})$. Entäo $X^{\prime}$ é um espaço NPL.

2) Se $K \vec{e}$ um espaço compacto (Hausdorff) não disperso então $[C(K)]^{\prime}$ è um espaço NPL.

3) $\operatorname{BV}([a, b], \mathbb{K}),(\|f\|=V[f]+|f(a)|)$ è um espaço NPL.

DEMONSTRACĀO - 1) Consideramos o espaço real $X_{\mathbb{R}}$ subjacente a $X$, que conterä um subespaço isomorfo $\ell_{1}(\mathbb{N}, \mathbb{R})$ e entäo $\left(X_{\mathbb{R}}\right)^{\prime}$ será NPL. Como consequência deste fato e da aplicação $g \in\left(x_{\mathbb{R}}\right)^{\prime} \rightarrow f(x)=g(x)-i g(i x) \in\left(x_{\mathbb{C}}\right)^{\prime}$ ser uma isometria (embora linêar somente sobre $\left.\left(X_{\mathbb{C}}\right)_{\mathbb{R}}^{\prime}\right)$, concluímos que $\left(X_{\mathbb{C}}\right)^{\prime}$ é NPL.

2) Se $K \vec{e}$ nã̃o disperso sabemos que $[C(K, \mathbb{R})]^{\prime}$ contẻm subespaço isomorfo a $\ell_{1}(\mathbb{N}, \mathbb{C})$ (veja [L-E], corolärio, pägina 1]4); 
aplicando 1), temos a tese.

3) Basta observar que, pelo teorema de representação de Riesz, BV([a,b], $\mathbb{K})$ contẻm subespaço isomorfoa $[\mathrm{C}([\mathrm{a}, \mathrm{b}], \mathbb{K})]^{\prime}$ e que $[a, b]$ não é disperso. CQD

Convëm observar que os espaços $\ell_{1}(\Gamma, \mathbb{K})$ de dimensão infinita ( $\Gamma$ conjunto infinito) não são reflexivos, pois contëm um espaço isomorfo a $\ell_{1}(\mathbb{N}, \mathbb{K})$, o qual não ë reflexivo. Surge então naturalmente a questão: "Existe um espaço reflexivo com a propriedade de Lebesgue?"

Exemplos de espaços reflexivos são os espaços de Lorentz, definidos em 2.b.8; quando $p>1$, entretanto:

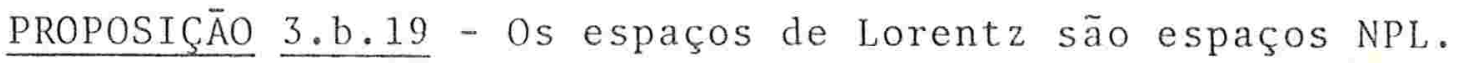
DEMONSTRAÇĀO - Faremos a demonstração para o caso de $[\mathrm{a}, \mathrm{b}]=$ $=[0,1]$. Dado d(w,p) um espaço de Lorentz, consideremos uma enumeração $\left\{r_{i}\right\}_{i=1}^{\infty}$ dos racionais de $[0,1]$. Seja

$$
f:[a, b] \rightarrow d(w, p)
$$

dada por

$$
f(x)=\left\{\begin{array}{l}
e_{i} \text { se } x=r_{i}, \text { onde } e_{i}=(0,0, \ldots, 0,1,0,0, \ldots) \\
0 \text { se } x \notin\left\{r_{i}\right\}_{i=1}^{\infty} .
\end{array}\right.
$$

E óbvio que $D(f)=[0,1]$; decorre que $u[D(f)]=1, e$ portanto, $f \notin D([0,1], d(w, p))$. Contudo, $f \in R([0,1], d(w, p))$ pois:

$$
\| \sum_{i=1}^{|d|} f\left(\varepsilon_{i}\right)\left[t_{i}-t_{i-1}\right]-0 \mid \leq\left(\left.|\Delta d|^{p_{w_{1}}+\left|\Delta d_{1}\right|^{p_{w_{2}}+\ldots+\mid \Delta d}|d|-1}\right|^{p_{w}}|d|-1\right)^{\frac{1}{p}} .
$$


Dado $\varepsilon>0$, tomemos $n_{0}$ tal que, se $n \geq n_{0}, w_{n_{0}}<\frac{\varepsilon^{p}}{2} \cdot$ Se $\Delta d<\sqrt[p]{\frac{\varepsilon^{p}}{2 \sum_{i=1}^{n_{0} w_{i}}}}$ vira $:\left\|\sum_{i=1}^{|d|} f\left(\varepsilon_{i}\right)\left[t_{i}-t_{i-1}\right]-0\right\| \leq\left(\frac{\varepsilon^{p}}{2}+\frac{\varepsilon^{p}}{2}\right)^{1 / p}=\varepsilon$. $\log 0, \int_{0}^{1} f(x) d x=0$.

Em relação aos espaços reflexivos, gostaríamos agora de chamar atenção para o seguinte: um espaço PL reflexivo não pode conter nenhum subespaço uniformemente convexo de dimensão infinita. (Pois senão, por 6 do corolärio 3.b.5, seria NPL).

Estudando os espaços reflexivos de dimensão infinita que não contém subespaços uniformemente convexos de dimensão infinita, encontramos um exemplo bastante simples devido a T.Figiel e W.B.Johnson (veja [2.b.9.]), que resolveu o prob1ema.

TEOREMA 3.b.20 - Existe um espaço reflexivo de dimensão infinita com a propriedade de Lebesgue.

DEMONSTRAÇĀO - Seja T o espaço definido no exemplo 2.b.9. Suponhamos que exista $f \notin I([a, b], T), f \in R([a, b], T)$. Como

$$
D(f)=\bigcup_{n=1}^{\infty} F_{n}, F_{n}=\left\{t / w(t, f) \geq \frac{1}{n}\right\} \text { e } u(D(f))>0,
$$

existe $n_{0}$ tal. que $u\left(F_{n_{0}}\right)=C>0$. Por outro lado, as funções $\mathrm{P}_{\mathrm{n}}: \mathrm{T} \rightarrow \mathbb{K}(\mathbb{K}=\mathbb{R}$ ou $\mathbb{C})$, dadas por $\mathrm{P}_{n}\left(\mathrm{a}_{1}, \mathrm{a}_{2}, \ldots, \mathrm{a}_{\mathrm{n}}, \ldots\right)=\mathrm{a}_{\mathrm{n}}$, 
são lineares continuas, e portanto $\mathrm{P}_{\mathrm{n}} \circ \mathrm{f} \in \mathrm{R}([\mathrm{a}, \mathrm{b}], \mathbb{K})$. Logo,

$$
u\left[D\left(P_{n} \circ f\right)\right]=0 \text {, e então, } u\left[\bigcup_{n=1}^{\infty} D\left(P_{n} \circ f\right)\right]=0 \text {. }
$$

Seja $d=\left\{a=t_{0}<t_{1}<\ldots<t_{m}=b\right\}$ uma partição de $[a, b]$, com $t_{i}-t_{i-1}=\frac{b-a}{m}, 1 \leq i \leq m$. Denotemos por $I_{1}, I_{2}, \ldots, I_{p}$ os intervalos de d que interceptam $\mathrm{F}_{\mathrm{n}_{0}}$ em um conjunto de medida não nula (1). E claro que $\frac{\mathrm{p}}{\mathrm{m}}(\mathrm{b}-\mathrm{a}) \geq \mathrm{C}\left(_{\infty}{ }^{*}\right)$, pois $\mathrm{u}\left[\mathrm{F}_{\mathrm{n}_{0}}\right]=\mathrm{C}>0$. Dado $\varepsilon>0$ escolhemos $x_{1} \in I_{1}{ }^{n F_{n_{0}}}, x_{1} \notin \bigcup_{n=1}^{\infty} D\left(P_{n} \circ f\right)$; então existe $y_{1} \in I_{1}$ tal que, se $e_{1}=f\left(x_{1}\right)-f\left(y_{1}\right)=\left(a_{1}, a_{2}^{1}, a_{3}^{1} \ldots a_{j}^{1} \ldots\right)$, temos:

a) $\left\|f\left(x_{1}\right)-f\left(y_{1}\right)\right\| \geq \frac{1}{2 n_{0}}$;

b) $\left\|\left(a_{1}^{1}, \ldots, a_{p+1}^{1}, 0,0, \ldots\right)\right\| \leq \frac{2}{2^{20}}$ (sendo $p$ o nümero de intervalos de (1)).

Seja $j_{1}>p+1$ o indice tal que

$$
\left\|\left(0,0,0, \ldots, 0, a_{J_{1}}^{1}, a_{J_{1}+1}^{1}, \ldots\right)\right\| \leq \frac{\varepsilon}{2^{21}} .
$$

Vamos agora a $I_{2}$ e tomamos $x_{2} \in I_{2} \cap F_{n_{0}}, x_{2} \notin \bigcup_{n=1}^{\infty} D\left(P_{n} \circ f\right)$ e $y_{2} \in I_{2}$ tal que, se $e_{1}=f\left(x_{2}\right)-f\left(y_{2}\right)=\left(a_{1}^{2}, a_{2}^{2}, \ldots, a_{j}^{2}, \ldots\right)$, temos:

a) $\left\|f\left(x_{2}\right)-f\left(y_{2}\right)\right\| \geq \frac{1}{2 n_{0}}$;

b) $\left\|\left(a_{1}^{2}, \ldots, a_{J_{1}}^{2}, 0,0, \ldots\right)\right\| \geq \frac{\varepsilon}{2^{22}}$.

Seja $j_{2}>j_{1}$ um indice tal que

$$
\left\|\left(0,0, \ldots, 0, a_{J_{2}}^{2}, a_{J_{2}+1}^{2}, \cdots\right)\right\| \leq \frac{\varepsilon}{2^{23}} .
$$


Em $I_{3}$ tomamos $x_{3} \in I_{3} \cap F_{n}, x_{3} \notin \bigcup_{n=1}^{\infty} D\left(P_{n}\right.$ of $)$ e $y_{3} \in I_{3}$ tal que, se $e_{3}=f\left(x_{3}\right)-f\left(y_{3}\right)=\left(a_{1}^{3}, \ldots, a_{j}^{3}, \ldots\right)$, temos:

a) $\left\|f\left(x_{3}\right)-f\left(y_{3}\right)\right\| \geq \frac{1}{2 n_{0}}$;

b) $\left\|\left(a_{1}^{3}, a_{2}^{3}, \ldots, a_{j_{2}}^{3}, 0,0,0, \ldots\right)\right\| \leq \frac{\varepsilon}{2^{24}}$, e seja $j_{3}>j_{2}$ um indice tal que $\left\|\left(0,0,0, \ldots 0, a_{J_{3}}^{3}, a_{J_{3}+1}^{3}, \ldots\right)\right\| \leq \frac{\varepsilon}{2{ }^{25}}$.

Procedendo assim sucessivamente, escolhemos veto-

res

$$
\begin{aligned}
& e_{1}=f\left(x_{1}\right)-f\left(y_{1}\right)=\left(a_{1}^{1}, a_{2}^{1}, \ldots, a_{j_{0}}^{1}, a_{p+2}^{1}, \ldots, a_{j_{1}}^{1}, a_{j_{1}+1}^{1}, \ldots \ldots \ldots \ldots . . .,\right. \\
& e_{2}=f\left(x_{2}\right)-f\left(y_{2}\right)=\left(a_{1}^{2}, a_{2}^{2}, \ldots \ldots . . . ., a_{j_{1}}^{2}, a_{j_{1}+1}^{2}, \ldots, a_{j_{2}}^{2}, \ldots \ldots . . .\right. \\
& \vdots \quad \vdots \\
& e_{p}=f\left(x_{p}\right)-f\left(y_{p}\right)=\left(a_{1}^{p}, a_{2}^{p}, \ldots \ldots \ldots \ldots \ldots \ldots, \ldots, a_{j_{p-1}}^{p}, a_{j}^{p}, \ldots\right),
\end{aligned}
$$

onde $j_{0}=p+1$.

$1 \leq \mathrm{i} \leq \mathrm{p}$, vem que

$$
\text { Fazendo } e_{i j_{i-1}}=\left(0,0,0, \ldots, 0, a_{j_{i-1}}^{i}, \ldots, a_{j_{1}-1}^{i}, 0,0, \ldots\right) \text {. }
$$

$$
\begin{aligned}
& e_{1}-e_{1 j_{0}}=\left(a_{1}^{1}, \ldots, a_{p+1}^{1}, 0,0, \ldots, 0, a_{j_{1}}^{1}, a_{j_{i}+1}^{1}, \ldots \ldots \ldots \ldots \ldots \ldots \ldots \ldots \ldots \ldots \ldots,\right. \\
& e_{2}-e_{2 j_{1}}=\left(a_{1}^{2}, \ldots, a_{p+1}^{2}, \ldots \ldots \ldots, a_{j_{1}}^{2}, 0,0, \ldots, 0, a_{j_{2}}^{2}, a_{j_{2}+1}^{2}, \ldots \ldots \ldots \ldots \ldots \ldots, \ldots, \ldots,\right. \\
& e_{p}-e_{p j_{p-1}}=\left(a_{1}^{p}, \ldots, a_{p+1}^{p}, \ldots \ldots \ldots \ldots \ldots, \ldots, \ldots, a_{j}^{p}, 0,0, \ldots, 0, a_{j}^{p}, a_{j}^{p}{ }_{p}^{p}+1, \ldots\right) .
\end{aligned}
$$

Logo, 


$$
\begin{aligned}
& \left\|\sum_{i=1}^{p} e_{i}\right\| \geq\left\|\sum_{i=1}^{p} e_{i j}\right\|-\left\|\sum_{i=1}^{p}\left(e_{i}-e_{i j}\right)\right\| \geq \\
& \geq\left\|\sum_{i=1}^{p} e_{i j}\right\|-\left[\frac{\varepsilon}{2^{20}}+\frac{\varepsilon}{2^{21}}+\ldots\right] \geq\left\|\sum_{i=1}^{p} e_{i j}\right\|-\frac{\varepsilon}{2^{19}} .
\end{aligned}
$$

Por outro 1ado, $\left\|e_{i j_{i-1}}\right\| \geq \frac{1}{2 n_{0}}-\frac{\varepsilon}{2^{19+2(i-1)}}$, e vale ainda que

$$
\left\|\sum_{i=1}^{p} e_{i j}\right\|=\max \left\{\max _{n \in \mathbb{N}}\left|a_{n}\right|, \frac{1}{2} \sup \left(\sum_{j=1}^{K}\left\|\sum_{n=p_{j}+1}^{P_{j+1}} a_{n} e_{n}\right\|,\right.\right.
$$

$\left.\left.\mathrm{K} \leq \mathrm{p}_{1}<\mathrm{p}_{2}<\ldots<\mathrm{p}_{\mathrm{K}+1}, \mathrm{~K}=1,2, \ldots\right)\right\}$.

Escolhendo $\mathrm{K}=\mathrm{p}$, obtemos:

$$
\left\|\sum_{i=1}^{p} e_{i j_{i-1}}\right\| \geq \frac{1}{2}\left(\sum_{i=1}^{p}\left\|e_{i j}\right\|\right) \geq \frac{1}{2}\left(\frac{p}{2 n_{0}}-\sum_{i=1}^{p} \frac{\varepsilon}{2^{19+2(i-1)}}\right)
$$

e então

$$
\left\|\sum_{i=1}^{p} e_{i}\right\| \geq \frac{1}{2}\left(\frac{p}{2 n_{0}}-\sum_{i=1}^{p} \frac{\varepsilon}{2^{19+2(i-1)}}\right)-\frac{\varepsilon}{2^{19}} \geq \frac{1}{2}\left[\frac{p}{2 n_{0}}-\frac{\varepsilon}{2}\right] .
$$

Feitas estas considerações, voltemos aos intervalos da divisão d. Para cada $1 \leq \mathrm{i} \leq \mathrm{m}$, tomemos

$$
\left\{\begin{array}{l}
\varepsilon_{i}=\varepsilon_{i}^{\prime}, \text { se }\left[t_{i-1}, t_{i}\right] \neq I_{i}, 1 \leq i \leq p . \\
\varepsilon_{i}=x_{i}, \varepsilon_{i}^{\prime}=y_{i}, \text { se }\left[t_{i-1}, t_{i}\right]=I_{i} \text {, para algum } i, 1 \leq i \leq p .
\end{array}\right.
$$

Temos que

$$
\left\|\sum_{i=1}^{|d|}\left[f\left(\varepsilon_{i}\right)-f\left(\varepsilon_{i}^{\prime}\right)\right] \frac{b-a}{m}\right\| \geq \frac{b-a}{m}\left[\frac{1}{2}\left(\frac{p}{2 n_{0}}-\frac{\varepsilon}{2}\right)\right]=
$$




$$
\begin{aligned}
& =\frac{1}{2}\left[\frac{p(b-a)}{2 m} \times \frac{1}{n_{0}}-\frac{\varepsilon(b-a)}{2 m}\right] \geq \frac{1}{2}\left[\frac{C}{2 n_{0}}-\frac{\varepsilon(b-a)}{2}\right]= \\
& =\frac{C}{4 n_{0}}-\frac{\varepsilon(b-a)}{4} .
\end{aligned}
$$

Tomando $\varepsilon \leq \frac{\mathrm{C}}{2 \mathrm{n}_{0}(\mathrm{~b}-\mathrm{a})}$ resulta:

$$
\left\|\sum_{i=1}^{|d|}\left[f\left(\varepsilon_{i}\right)-f\left(\varepsilon_{i}^{\prime}\right)\right] \frac{b-a}{m}\right\| \geq \frac{c}{8 n_{0}},
$$

e então, pela proposição 1.a.5, fÆR([a,b],T). CQD

\section{$\underline{3 . b .21}-\underline{\text { Resumo Gera1 }}$}

Dado um espaço de Banach $X$ de dimensão infinita,os problemas $[\mathrm{P}-1],[\mathrm{P}-2]$ e $[\mathrm{P}-3]$ permitem dar um quadro das situações para os conjuntos $B V([a, b], X), B W([a, b], X), G([a, b], X)$, $D([a, b], X)$ e $R([a, b], X)$.

1․ caso - X não contëm subespaço isomorfo a $C_{0}(\mathbb{N})$. Temos duas possibilidades descritas pelas figuras a) e b), que correspondem aos casos de espaços NPL e espaços PL. a) NPL

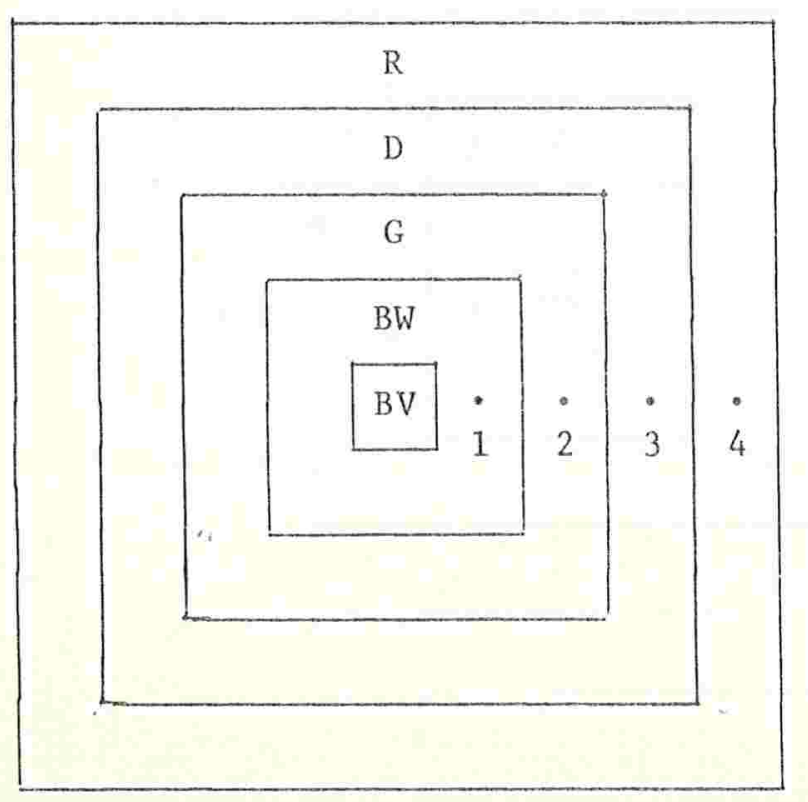

b) $\mathrm{PL}$

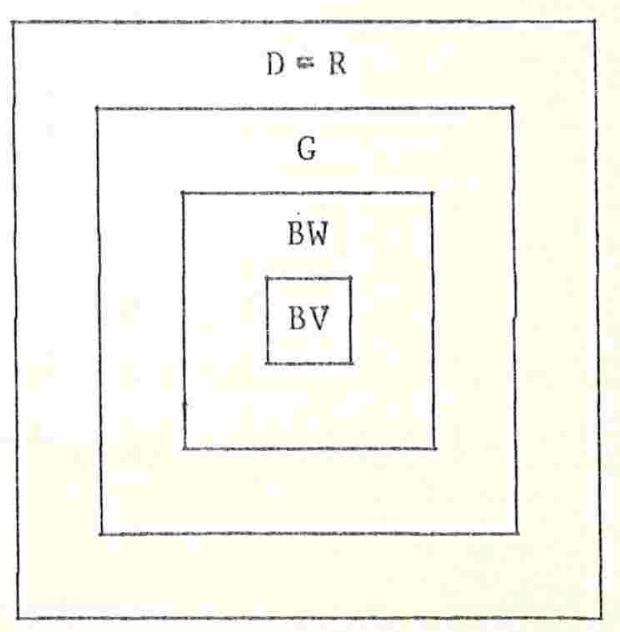


o caso a) ocorre, por exemplo, para os espaços uniformemente convexos. o caso b) ocorre, por exemplo, para $\ell_{1}(\mathbb{N})$ e para espaços reflexivos, como o espaço $T$ do teorema 3.b. 20 .

E imediato que as inclusões nos casos a) e b) são pröprias, pois:

1) $\operatorname{BV}([a, b], x) q \operatorname{BW}([a, b], X)$ (veja problema 1).

2) $B W([a, b], X) \& G([a, b], X)$. (Basta tomar una $f:[a, b] \rightarrow Y \subset X$, onde $\operatorname{dim} Y=1, f \in G([a, b], Y) e \operatorname{f\notin BV}([a, b], Y)=\operatorname{BW}([a, b], Y)$.

3) $G([a, b], X) \notin D([a, b], X)$. (o mesmo raciocínio que em 2).

4) $D([a, b], X) \varsubsetneqq R([a, b], X)$. (Para o caso a), imediato pela definição de espaço NPL).

$\underline{29}$ caso - X contêm subespaço isomorfo a $C_{0}(\mathbb{N})$. Temos agora uma única possibilidade descrita pela figura abaixo.

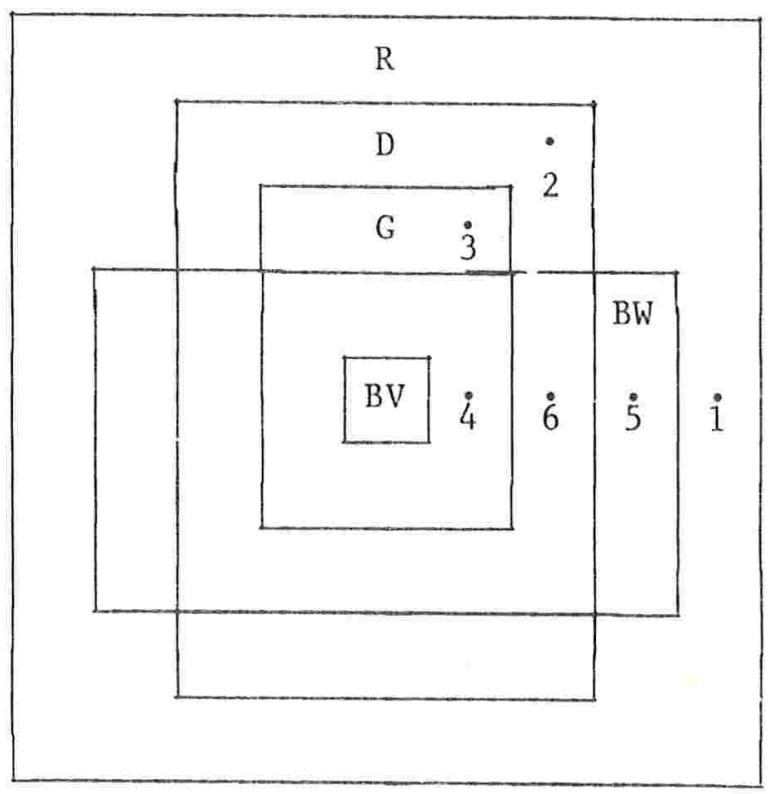


Os exemplos seguintes mostram sera configuração acima verdadeira.

1) Seja $\left\{r_{i}\right\}_{i=1}^{\infty}$ uma enumeração dos racionais de $[a, b]$,e definimos

$$
f(x)=\left\{\begin{array}{l}
f_{i}, \text { se } x=r_{i} \\
0, \text { se } x \notin\left\{r_{i}\right\}_{i=1}^{\infty}
\end{array},\right.
$$

onde $f_{i}=\left(\frac{1}{i}, 0,0, \ldots, 0,1,0,0, \ldots\right)$.

2) $f:[a, b] \rightarrow c_{0}(\mathbb{N}), f(x)=x_{C}(x) \cdot e$, onde $x_{C}:[0,1] \rightarrow \mathbb{R}$ $\vec{e}$ função caracteristica do conjunto triädico de Cantor e $\mathrm{e} \in \mathcal{C}_{0}(\mathbb{N}), e \neq 0$.

3) $\mathrm{f}:[\mathrm{a}, \mathrm{b}] \rightarrow \mathrm{C}_{0}(\mathbb{N}), \mathrm{f}(\mathrm{x})=\mathrm{h}(\mathrm{x}) \cdot \mathrm{e}, \operatorname{com} \mathrm{h}:[\mathrm{a}, \mathrm{b}] \rightarrow \mathbb{R}$ ta 1 que $h \notin B V([a, b], \mathbb{K}), h \in G([a, b], \mathbb{K})$ e e $\in_{0}(\mathbb{N}), e \neq 0$.

4) Considere $f:[a, b] \rightarrow C_{0}(\mathbb{N})$ a função de demonstração do teorema $1 . g \cdot 1$ (problema 1$)$.

5) Considere $f:[a, b] \rightarrow c_{0}(\mathbb{N})$, definida em 1.e.2.

6) Seja $\left\{r_{i}\right\}_{i=1}^{\infty}$ um conjunto enumerävel contido no conjunto de Cantor e denso neste conjunto. Seja $f:[a, b] \rightarrow C_{0}(\mathbb{N})$, dada por

$$
f(x)=\left\{\begin{array}{l}
e_{i}, \text { se } x=r_{i} \\
0, \text { se } x \notin\left\{r_{i}\right\}_{i=1}^{\infty}
\end{array}, \text { onde } e_{i}=(0,0, \ldots, 0,1,0,0, \ldots) .\right.
$$

E imediato que $D(f)=C$ (Conjunto de Cantor) e $u[D(f)]=0$. 
$\log o, f \in D\left([a, b], C_{0}(\mathbb{N})\right)$, e um cälculo simples mostra que

$$
f \in B W\left([a, b], c_{0}(\mathbb{N})\right) \text { e } f \notin G\left([a, b], c_{0}(\mathbb{N})\right)
$$

$\underline{3 . b .22}-\underline{\text { Problemas em aberto }}$

Vamos agora relacionar algumas questões ligadas ainda ao problema 3 e à solução do problema 4.

1) Se X é um espaço de Shurr (veja definição 2.b.10) então vale que $\mathrm{X}$ tem a propriedade de Lebesgue?

2) "Caracterizar" os espaços reflexivos $X$ tais que

$$
D([a, b], x)=R([a, b], x) \text {. }
$$

3) "Caracterizar" os espaços de Banach em que $D([a, b], X)=$

$$
=R([a, b], x) \text {. }
$$

E claro que, para começarmos a abordar o problema 4 (vide introdução), teríamos de resolver a questão 3 acima. Tais questões esclareceriam definitivamente a teoria de integração de Riemann para funções definidas em $[a, b]$ e a valores num espaço de Banach.

\section{3.c-APLICAÇŌES DOS RESULTADOS}

Vimos que $f \in B W([a, b], X)$. se e só se $x^{\prime} \operatorname{of} \in B N([a, b], \mathbb{K})=$ $=\operatorname{BV}([a, b], \mathbb{K})$, para toda $x^{\prime} \in X^{\prime}$ (teorema 1.d.6).

E natural perguntar se resultados análogos não são verdadeiros para as classes $B V([a, b], X), G([a, b], X), D([a, b], X)$ e $R([a, b], x)$.

A resposta a estas questões è simples e pode ser descrita pela: 
$\underline{\text { PROPOSICĀO }} \underline{3 . c \cdot 1}$ - Seja $X$ um espaço de Banach e $f:[a, b] \rightarrow X$.

1) São equivalentes:

a) Se $x^{\prime} \circ f \in B V([a, b], \mathbb{K})$, para toda $x^{\prime} \in X^{\prime}$, então

$$
\operatorname{feBV}([a, b], x) \text {. }
$$

b) $x$ tem dimensão finita.

2) São equivalentes:

a) $x^{\prime} \circ f \in G([a, b], \mathbb{K})$, para toda $x^{\prime} \in X^{\prime}$, implica

$$
f \in G([a, b], x) \text {. }
$$

b) $x^{\prime} \circ f \in G([a, b], \mathbb{K})$, para toda $x^{\prime} \in X^{\prime}$, implica

$$
f \in D([a, b], x) \text {. }
$$

c) $X \ddot{e}$ um espaço de Schurr.

3) Temos as implicações: a) $\Longrightarrow$ b) $\Longrightarrow$ c) $\Longrightarrow$ d), onde

a) $x$ é Schurr.

b) $x^{\prime} \circ f \in R([a, b], \mathbb{K})$, para toda $x^{\prime} \in X^{\prime}$, implica

$$
f \in R([a, b], x) \text {. }
$$

c) $x^{\prime} \circ f \in G([a, b], \mathbb{K})$, para toda $x^{\prime} \in X^{\prime}$, implica

$$
f \in R([a, b], x) \text {. }
$$

d) $\mathrm{X}$ e Schurr ou $\mathrm{X}$ não tem a propriedade de Lebesgue.

4) São equivalentes:

a) $x^{\prime} \circ f \in R([a, b], \mathbb{K})$, para toda $x^{\prime} \in X^{\prime}$, implica

$$
f \in D([a, b], x) \text {. }
$$

b) $x \vec{e}$ Schurr e tem a propriedade de Lebesgue.

5) Se $x \vec{e}$ im espaço que não tem a propriedade de Lebesgue então existe $f \notin D([a, b], X)$ tal que $x^{\prime} \circ f \in D([a, b], \mathbb{K})$, 
para toda $x^{\prime} \in X^{\prime}$.

6) Se $x$ é um espaço de dimensão infinita, reflexivo,com a propriedade de Lebesgue, existe $f(R([a, b], X)$ tal que $x^{\prime} \circ f \in G([a, b], \mathbb{K}) \subset D([a, b], \mathbb{K})=R([a, b], \mathbb{K})$, para toda $x^{\prime} \in X^{\prime}$. (o teorema 3.b.20 mostra a existência dos espaços a que se referem 6 acima).

$\underline{\text { DEMONSTRACĀO }}$ - 1) a) $\Longrightarrow$ b) Se a dimensão de X for infinita tomemos $f \in B W([a, b], X), f \notin B V([a, b], X)$ (teorema 1.g.1).Assim, $x^{\prime} \circ f \in B V([a, b], \mathbb{K})$, para toda $x^{\prime} \in X^{\prime}$ (teorema 1.d.6), que contraria a hipôtese, e portanto, a dimensão de $x$ é finita.

b) $\Longrightarrow$ a) Demonstração imediata.

2) a) $\Longrightarrow$ b) Demonstração imediata.

b) $\Longrightarrow$ c) Se X não é um espaço de Schurr, existe uma sequência $\left\{x_{n}\right\}_{n=1}^{\infty}, x_{n} \frac{\text { francamente }}{n \rightarrow \infty} 0$, com $x_{n}$ não convergente em norma e ta1 que $\left\|x_{n}\right\| \geq \delta>0$.

Considere $\left\{r_{i}\right\}_{i=1}^{\infty}$ uma enumeração dos racionais de $[a, b]$ e $f:[a, b] \rightarrow x$, definida por

$$
f(x)=\left\{\begin{array}{l}
x_{i} \text { se } x=r_{i} \\
0 \text { se } x \notin\left\{r_{i}\right\}_{i=1}^{\infty}
\end{array}\right.
$$

E imediato que $x^{\prime} \circ f \in G([a, b], \mathbb{K})$, para toda $x^{\prime} \in X^{\prime}$, e

$$
f \notin D([a, b], x) \text {. }
$$

c) $\Longrightarrow$ a) Por hipōtese, $x^{\prime} \circ f \in G([a, b], \mathbb{K})$, para toda $x^{\prime} \in X^{\prime}$. 
Assim, se $t_{n} \rightarrow t$ então $\left(x^{\prime} \circ f\right)\left(t_{n}\right)$ converge em $\mathbb{C}$, para toda $x^{\prime} \in X^{\prime}$. De 2 da proposição 2.b.11, sabemos que X é fracamente sequencialmente completo; logo existe $x_{0} \in X$ tal que

$$
\left(x^{\prime} \circ f\right)\left(t_{n}\right) \stackrel{n \rightarrow \infty}{\longrightarrow} x^{\prime}\left(x_{0}\right)
$$

e ainda, como tal espaço é de Schurr, $f\left(t_{n}\right) \stackrel{n \rightarrow \infty}{\stackrel{n}{\prime \prime}} x_{0}$. Assim,

$$
\mathrm{fEG}([\mathrm{a}, \mathrm{b}], \mathrm{X}) \text {. CQD }
$$

3) a) $\Longrightarrow$ b) Seja $d_{n}$ uma sequência de divisões tal que $\lim _{n \rightarrow \infty} \Delta d_{n}=0$. Seja $s$ o conjunto de sequências tomando-se

$$
s_{n}=\sum_{n=1}^{\left|d_{n}\right|} f\left(\varepsilon_{i}\right)\left[t_{i}-t_{i-1}\right] \text {, }
$$

e fixando-se $\varepsilon_{i} \in\left[t_{i-1}, t_{i}\right]$ de forma arbiträria. Como

$$
x^{\prime} \circ f \in R([a, b], \mathbb{K})
$$

segue que, dada uma sequência $s_{n} \in S,\left\{x^{\prime}\left(s_{n}\right)\right\}_{n=1}^{\infty}$ converge em $\mathbb{K}$; como X é Schurr (portanto, fracamente sequenciamente completo), concluímos que $s_{n} \rightarrow x_{0} \in X$. Por outro lado, se tomarmos $s_{n}^{\prime} \in S$, também concluímos que. $s_{n}^{\prime} \rightarrow x_{0}^{\prime}$, e ë imediato que $x_{0}=x_{0}^{\prime}$. Da mesma forma, se considerarmos $d_{n}^{\prime} \operatorname{com} \underset{n \rightarrow \infty}{\lim } \Delta d_{n}^{\prime}=0$, tambëm teremos que as sequências de $S^{\prime}$ convergem a $x_{0}$. Demonstraremos que existe

$$
\int_{a}^{b} f(t) d t=x_{0} .
$$

Caso conträrio, existe $\varepsilon>0$ e uma sequência da divisão $\mathrm{d}_{n}$ 
com $\lim _{n \rightarrow \infty} \Delta d_{n}=0$ tal que

$$
\left\|\sum_{i=1}^{\left|d_{n}\right|} f\left(\varepsilon_{i}\right) \Delta t_{i}-x_{0}\right\| \geq \varepsilon,
$$

para uma dada escolha de $\varepsilon_{i} \in\left[t_{i-1}, t_{i}\right]$, em cada divisão $d_{n}$, o que contraria a afirmação anterior.

b) $\Rightarrow$ c) Imediata.

c) $\Longrightarrow$ d) Se $X$ não fosse Schurr e tivesse a propriedade de Lebesgue, a função

$$
f(x)=\left\{\begin{array}{l}
x_{i} \text { se } x=r_{i} \\
0 \text { se } x \notin\left\{r_{i}\right\}_{i=1}^{\infty},
\end{array}\right.
$$

definida na demonstração de 2, b) $\Longrightarrow$ c), teria a propriedade de $f \notin D([a, b], x) ; \log 0 \operatorname{f\notin R}([a, b], x) \operatorname{mas} x^{\prime} \circ f \in G([a, b], \mathbb{K})$, para toda $x^{\prime} \in X^{\prime}$.

4) a) $\Longrightarrow$ b) $x$ é Schurr pois, caso conträrio, f, definida na demonstração de 2 da proposição b) $\Rightarrow$ c), contraria a hipôtese.

$X$ tem a propriedade de Lebesgue senão existiria $f \in R([a, b], x) ; f \notin D([a, b], x)$ e $x^{\prime} \circ f \in R([a, b], \mathbb{K})$, para toda $x^{\prime} \in X^{\prime}$, - que contraria novamente a hipótese.

b) $\Longrightarrow$ a) Se $X \hat{e} \operatorname{Schurr}$ e $x^{\prime} \operatorname{of} \in R([a, b], \mathbb{K})$, para toda $x^{\prime} \in X^{\prime}$, entäo, por $3 b)$, $f \in R([a, b], x)$, mas $x$ tem a propriedade de Lebesgue; $\log 0, \mathrm{f} \in D([\mathrm{a}, \mathrm{b}], \mathrm{x})$. 
5) Consequência imediata de 4 .

6) Consequência imediata de 4 (tomando-se o cuidado de verificar, na demonstração, que $\left.x^{\prime} \circ f \in G([a, b], \mathbb{K})\right)$.

CQD

OBSERVAÇĀO 3 3.c.2 - Depois de terminada a elaboração desta tese tomamos conhecimento através do Mathematical Reviews do artigo: On Riemann integrability conditions for functions with values in Banach space, A.S. Nem irovsky M. Yu Ochan, R. Redjouani, Vesnik Moskovo Universiteta n 4, 1972, onde se encontra uma outra demonstração do teorema 3.b.12. 
[B-P] - Bessaga,C.\& Pelczynski,A., On bases and unconditional convergence of series in Banach spaces, Studia Mathematica, 17(2): $151-164,1958$.

[C] - Clarkson,J.A., Uniformly convex spaces, Transactions of the American Mathematical Socicty, 40(3): 396-414, 1936.

[D] - Dieudonne, J., Foundations of modern analysis, New York, Academic Press, 1960, 361p. (Pure and Applied Mathematics, 10).

[D-S] - Dunford,N.\& Schwartz, J.,Linear operators, New York, Interscience, 1958-63, 3v., (Pure and Applied Mathematics).

[D-R] - Dvoretzky,A.\& Rogers,C.A., Absolute and unconditional convergence in normed linear spaces, Proceedings of the National Academy of Sciences, 36: 192-197, 1950.

[E-P] - Enflo,P., A counterexample to the approximation problem in Banach spaces, Acta Mathematica, 130: 309-317, 1973.

[F-J] - Figie1,T.\& Johnson,W.B., A uniformly convex Banach space which contains no $l_{p}$, Compositio Mathematica, 29(2):179-190,1974.

$[R-H]$ - Haydon, R., On Banach spaces which contain $\ell^{1}(\tau)$ and types of measures on compact spaces, Israel Journal of Mathematics, $28(4): 313-324,1977$.

[H-S] - Hewitt,E.छ Stromberg,K., Real and abstract analysis: a modern treatment of the theory of a real variable, Berlin, Springer, 1969, 476p. 
[H] - Hildebrandt,T.H., Introduction to the Theory of Integration, New York, Academic Press, 1963, 385p. (Pure and Applied Mathematics, 13).

[H-1] - Hönig, C.S., The abstract Riemann-Stieltjes integral and its applications to linear differential equations with generalized boundary conditions, São Paulo, Instituto de Matemätica e Estatística da Úniversidade de São Paulo,1973,135p. (Notas do INE-USP).

[H-4] - Hönig, C.S., Anälise funcional e aplicações, São Paulo, Instituto de Matemätica e Estatística da Universidade de São Pau$10,1970,2 \mathrm{v}$.

[H-5] - Hönig, C.S., Compactifying subspaces, In: 7 Seminärio Brasileiro de Anälise, Recife, 1978, Trabalhos apresentados, Rio de Janeiro, SBM, 1978, p.73-86.

[H-3] - Hönig, C.S., Functional analys is and integro-differential equations with linear constraints, Rio de Janeiro, SBM, 1974, $204 \mathrm{p}$.

[H-2] - Honig, C.S., Open problems in the theory of differential equations with linear constraints, In: Semana de Anālise Funcional Não Linear, São Paulo, 1973, Atas, Rio de Janeiro, SBM, 1974, 268p. (Coleção Atas, 5) .

[RC-2] - James,R.C., Bases and reflexivity of Banach spaces, Annals of Mathematics, 52(3): 518-527, 1950.

[RC-5] - James,R.C., Characterizations of reflexivity, Studia Mathematica, 23(3): 205-216, 1964.

[RC-4] - James, R.C., A counterexample for a sup theorem in normed spaces, Israel Journal of Mathematics, 9(4): 511-512, 1971.

[RC-3] - James, C.R., A non-reflexive Banach space isometric with its second conjugate space, Proceedings of the National Academy of Sciences, 37: 174-177, 1951. 
[RC-1] - James,R.C., Super-reflexive spaces with bases, Pacific Journal of Mathematics, 41(2): 409-419, 1972.

[K-G] - Kothe,G., Topological vector spaces, Ber1in, Springer, 1969,v. (Die Grundlehren der Mathematischen Wissenschaften, 159).

[L-E] - Lacey,H.E., The isometric theory classical Banach spaces, Berlin, Springer, 1974, 270p. (Die Grundlehren der Mathematischen Wissenschaften, 208).

[L-T] - Lindenstrauss, J.\& Tzafriri,L., Classical Banach Spaces I, Ber1in, Springer, 1977, 190p. (ergebnisse der Mathematik und ihrer Grenzgebiete, 92).

[L] - Lorentz,G.G., Some new functional spaces, Annals of Mathematics, $51(1): 37-55,1950$.

[M-T] - Marti,J.T., Introduction to the Theory of Bases, Berlin, Springer, $1969,149 \mathrm{p}$.

[O-R] - Ode11,E.\& Rosenthal,H.P., A double-dual characterization of separable Banach spaces containing $\ell_{1}$, Israel Journal of Mathematics, 20(3): 375-384, 1975.

[P-S] - Pelczinzki,A. \& Semadeni,Z ., Spaces of continuous funtions III, Studia Mathematica, 18(2): 211-222, 1959.

[R] - Rosenthal,H.P., Point-wise compact subsets of the first Baire class, American Journal of Mathematics, 99(2):362-378,1977.

[S-I] - Singer, I., Bases in Banach spaces I, Ber1in, Springer, 1970, $668 \mathrm{p}$. (Die Grundlehren der Mathematischen Wissenschaften, 154).

[Y] - Yosida,K., Functional Analysis, Berlin, Springer, 1965, 458p., (Die Grundlehren der Mathematischen Wissenchaften, 123).

[Z] - Zygmund,A., Trigonometric series, 2.ed., Cambridge, University Press, $1959,2 \mathrm{v}$. 